\title{
CONSTRAINTS ON THE GALACTIC POPULATION OF TeV PULSAR WIND NEBULAE USING FERMI LARGE AREA TELESCOPE OBSERVATIONS
}

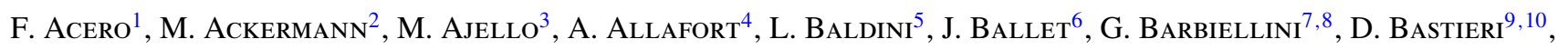
K. Bechtol ${ }^{4}$, R. Bellazzini ${ }^{11}$, R. D. Blandford ${ }^{4}$, E. D. Bloom ${ }^{4}$, E. Bonamente ${ }^{12,13}$, E. Bottacini ${ }^{4}$, T. J. BRandt ${ }^{1}$, J. Bregeon ${ }^{11}$, M. Brigida ${ }^{14,15}$, P. Bruel ${ }^{16}$, R. Buehler ${ }^{4}$, S. Buson ${ }^{9}, 10$, G. A. Caliandro ${ }^{17}$, R. A. Cameron ${ }^{4}$, P. A. CaraveO ${ }^{18}$, C. Cecchi $^{12,13}$, E. Charles ${ }^{4}$, R. C.G. Chaves ${ }^{6}$, A. CheKhtman ${ }^{19,58}$, J. Chiang ${ }^{4}$, G. Chiaro ${ }^{10}$, S. Ciprini ${ }^{20,21}$, R. Claus ${ }^{4}$, J. Cohen-Tanugi ${ }^{22}$, J. Conrad ${ }^{23,24,25,59}$, S. Cutini ${ }^{20,21}$, M. Dalton ${ }^{26,60}$, F. D'Ammando ${ }^{27}$, F. De Palma ${ }^{14,15}$, C. D. Dermer ${ }^{28}$, L. Di Venere ${ }^{4}$, E. do Couto e Silva ${ }^{4}$, P. S. Drell ${ }^{4}$, A. Drlica-Wagner ${ }^{4}$, L. Fallettir ${ }^{22}$, C. Favuzzi ${ }^{14,15}$, S. J. Fegan ${ }^{16}$,

E. C. Ferrara ${ }^{1}$, W. B. Focke ${ }^{4}$, A. FrancKowiak ${ }^{4}$, Y. Fukazawa ${ }^{29}$, S. FunK ${ }^{4}$, P. Fusco ${ }^{14,15}$, F. Gargano ${ }^{15}$, D. Gasparrini ${ }^{20,21}$, N. Giglietto ${ }^{14,15}$, F. Giordano ${ }^{14,15}$, M. Giroletti ${ }^{27}$, T. Glanzman ${ }^{4}$, G. Godfrey ${ }^{4}$, T. GréGoire ${ }^{30,31}$, I. A. Grenier ${ }^{6}$, M.-H. Grondin ${ }^{30,31}$, J. E. Grove ${ }^{28}$, S. Guiriec ${ }^{1}$, D. Hadasch ${ }^{17}$, Y. Hanabata ${ }^{29}$, A. K. Harding ${ }^{1}$, M. HaYashida ${ }^{4,32}$,

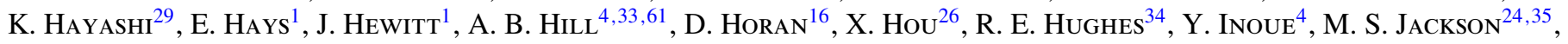

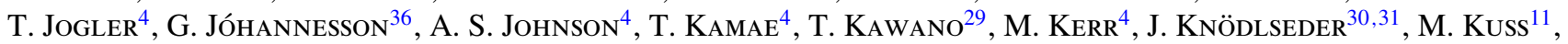

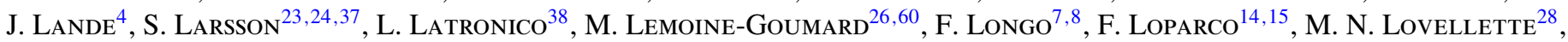
P. Lubrano ${ }^{12,13}$, M. Marelli ${ }^{18}$, F. Massaro ${ }^{4}$, M. MaYer ${ }^{2}$, M. N. Mazziotta ${ }^{15}$, J. E. McEneri ${ }^{1,39}$, J. Mehauli ${ }^{26,60}$, P. F. Michelson ${ }^{4}$, W. Mitthumsiri ${ }^{4}$, T. Mizuno ${ }^{40}$, C. Monte ${ }^{14,15}$, M. E. Monzani ${ }^{4}$, A. Morselli ${ }^{41}$, I. V. Moskalenko ${ }^{4}$, S. Murgia ${ }^{4}$, T. Nakamori ${ }^{42}$, R. Nemmen ${ }^{1}$, E. Nuss ${ }^{22}$, T. Ohsugi ${ }^{40}$, A. OKumura ${ }^{4,43}$, M. Orienti ${ }^{27}$, E. Orlando ${ }^{4}$, J. F. Ormes ${ }^{44}$, D. Paneque ${ }^{4,45}$, J. H. Panetta ${ }^{4}$, J. S. Perkins ${ }^{1,46,47,48}$, M. Pesce-Rollins ${ }^{11}$, F. Piron ${ }^{22}$, G. Pivato ${ }^{10}$, T. A. Porter ${ }^{4}$,

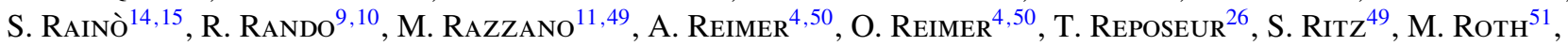
R. Rousseau ${ }^{26,60}$, P. M. Saz Parkinson ${ }^{49}$, A. Schulz ${ }^{2}$, C. Sgrò ${ }^{11}$, E. J. Siskind ${ }^{52}$, D. A. Smith ${ }^{26}$, G. SPandre ${ }^{11}$, P. SPinelli ${ }^{14,15}$, D. J. Suson ${ }^{53}$, H. TAKAhashi ${ }^{29}$, Y. TAKeuchi ${ }^{42}$, J. G. ThaYeR ${ }^{4}$, J. B. ThaYer ${ }^{4}$, D. J. Thompson ${ }^{1}$, L. Tibaldo $^{4}$, O. Tibolla ${ }^{54}$, M. Tinivella ${ }^{11}$, D. F. Torres ${ }^{17,55}$, G. Tosti ${ }^{12,13}$, E. Troja ${ }^{1,62}$, Y. UchiYama ${ }^{4}$, J. Vandenbroucke ${ }^{4}$,

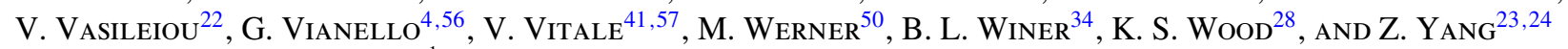
${ }^{1}$ NASA Goddard Space Flight Center, Greenbelt, MD 20771, USA

2 Deutsches Elektronen Synchrotron DESY, D-15738 Zeuthen, Germany

${ }^{3}$ Space Sciences Laboratory, University of California, 7 Gauss Way, Berkeley, CA 94720-7450, USA

${ }^{4}$ W. W. Hansen Experimental Physics Laboratory, Kavli Institute for Particle Astrophysics and Cosmology, Department of Physics and SLAC National Accelerator Laboratory, Stanford University, Stanford, CA 94305, USA; funk@ slac.stanford.edu, joshualande@ gmail.com

${ }^{5}$ Università di Pisa and Istituto Nazionale di Fisica Nucleare, Sezione di Pisa, I-56127 Pisa, Italy

${ }^{6}$ Laboratoire AIM, CEA-IRFU/CNRS/Université Paris Diderot, Service d'Astrophysique, CEA Saclay, F-91191 Gif sur Yvette, France

${ }^{7}$ Istituto Nazionale di Fisica Nucleare, Sezione di Trieste, I-34127 Trieste, Italy ${ }^{8}$ Dipartimento di Fisica, Università di Trieste, I-34127 Trieste, Italy

${ }^{9}$ Istituto Nazionale di Fisica Nucleare, Sezione di Padova, I-35131 Padova, Italy

${ }^{10}$ Dipartimento di Fisica e Astronomia “G. Galilei," Università di Padova, I-35131 Padova, Italy

${ }^{11}$ Istituto Nazionale di Fisica Nucleare, Sezione di Pisa, I-56127 Pisa, Italy

12 Istituto Nazionale di Fisica Nucleare, Sezione di Perugia, I-06123 Perugia, Italy

${ }^{13}$ Dipartimento di Fisica, Università degli Studi di Perugia, I-06123 Perugia, Italy

${ }^{14}$ Dipartimento di Fisica "M. Merlin" dell'Università e del Politecnico di Bari, I-70126 Bari, Italy

${ }^{15}$ Istituto Nazionale di Fisica Nucleare, Sezione di Bari, 70126 Bari, Italy

${ }^{16}$ Laboratoire Leprince-Ringuet, École polytechnique, CNRS/IN2P3, F-91128 Palaiseau, France

${ }^{17}$ Institut de Ciències de l'Espai (IEEE-CSIC), Campus UAB, E-08193 Barcelona, Spain

${ }^{18}$ INAF-Istituto di Astrofisica Spaziale e Fisica Cosmica, I-20133 Milano, Italy

${ }^{19}$ Center for Earth Observing and Space Research, College of Science, George Mason University, Fairfax, VA 22030, USA

${ }^{20}$ Agenzia Spaziale Italiana (ASI) Science Data Center, I-00044 Frascati (Roma), Italy

${ }^{21}$ Istituto Nazionale di Astrofisica-Osservatorio Astronomico di Roma, I-00040 Monte Porzio Catone (Roma), Italy

${ }^{22}$ Laboratoire Univers et Particules de Montpellier, Université Montpellier 2, CNRS/IN2P3, F-34095 Montpellier, France

${ }^{23}$ Department of Physics, Stockholm University, AlbaNova, SE-106 91 Stockholm, Sweden

${ }^{24}$ The Oskar Klein Centre for Cosmoparticle Physics, AlbaNova, SE-106 91 Stockholm, Sweden

${ }^{25}$ The Royal Swedish Academy of Sciences, Box 50005, SE-104 05 Stockholm, Sweden

${ }^{26}$ Université Bordeaux 1, CNRS/IN2p3, Centre d'Études Nucléaires de Bordeaux Gradignan,

F-33175 Gradignan, France; lemoine@cenbg.in2p3.fr, rousseau@cenbg.in2p3.fr

${ }^{27}$ INAF Istituto di Radioastronomia, I-40129 Bologna, Italy

${ }^{28}$ Space Science Division, Naval Research Laboratory, Washington, DC 20375-5352, USA

${ }^{29}$ Department of Physical Sciences, Hiroshima University, Higashi-Hiroshima, Hiroshima 739-8526, Japan ${ }^{30}$ CNRS, IRAP, F-31028 Toulouse cedex 4, France

${ }^{31}$ GAHEC, Université de Toulouse, UPS-OMP, IRAP, F-31028 Toulouse, France

32 Department of Astronomy, Graduate School of Science, Kyoto University, Sakyo-ku, Kyoto 606-8502, Japan

${ }_{33}$ School of Physics and Astronomy, University of Southampton, Highfield, Southampton SO17 1BJ, UK

${ }^{34}$ Department of Physics, Center for Cosmology and Astro-Particle Physics, The Ohio State University, Columbus, OH 43210, USA

35 Department of Physics, Royal Institute of Technology (KTH), AlbaNova, SE-106 91 Stockholm, Sweden

${ }^{36}$ Science Institute, University of Iceland, IS-107 Reykjavik, Iceland

${ }^{37}$ Department of Astronomy, Stockholm University, SE-106 91 Stockholm, Sweden

${ }^{38}$ Istituto Nazionale di Fisica Nucleare, Sezione di Torino, I-10125 Torino, Italy

${ }^{39}$ Department of Physics and Department of Astronomy, University of Maryland, College Park, MD 20742, USA

${ }^{40}$ Hiroshima Astrophysical Science Center, Hiroshima University, Higashi-Hiroshima, Hiroshima 739-8526, Japan

${ }^{41}$ Istituto Nazionale di Fisica Nucleare, Sezione di Roma “Tor Vergata,” I-00133 Roma, Italy 


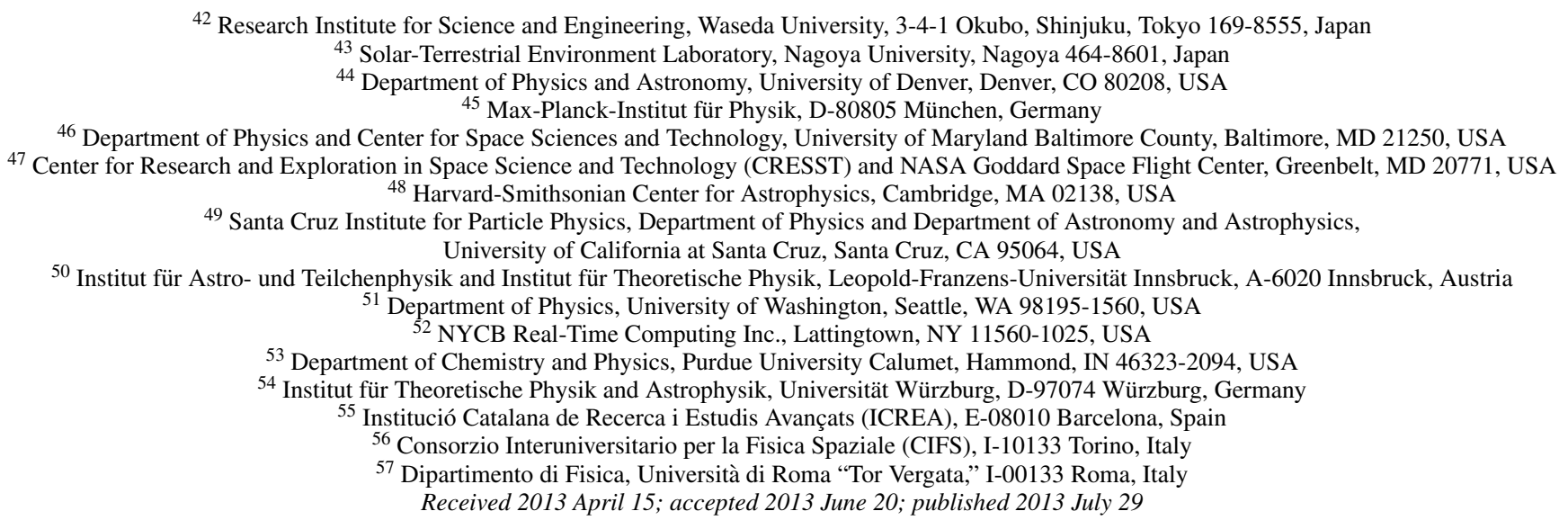

\begin{abstract}
Pulsar wind nebulae (PWNe) have been established as the most populous class of $\mathrm{TeV} \gamma$-ray emitters. Since launch, the Fermi Large Area Telescope (LAT) has identified five high-energy (100 MeV $<E<100 \mathrm{GeV}) \gamma$-ray sources as PWNe and detected a large number of PWN candidates, all powered by young and energetic pulsars. The wealth of multi-wavelength data available and the new results provided by Fermi-LAT give us an opportunity to find new PWNe and to explore the radiative processes taking place in known ones. The $\mathrm{TeV} \gamma$-ray unidentified (UNID) sources are the best candidates for finding new PWNe. Using 45 months of Fermi-LAT data for energies above $10 \mathrm{GeV}$, an analysis was performed near the position of $58 \mathrm{TeV}$ PWNe and UNIDs within $5^{\circ}$ of the Galactic plane to establish new constraints on PWN properties and find new clues on the nature of UNIDs. Of the 58 sources, 30 were detected, and this work provides their $\gamma$-ray fluxes for energies above $10 \mathrm{GeV}$. The spectral energy distributions and upper limits, in the multi-wavelength context, also provide new information on the source nature and can help distinguish between emission scenarios, i.e., between classification as a pulsar candidate or as a PWN candidate. Six new GeV PWN candidates are described in detail and compared with existing models. A population study of GeV PWN candidates as a function of the pulsar/PWN system characteristics is presented.
\end{abstract}

Key word: gamma rays: general

Online-only material: color figures

\section{INTRODUCTION}

Since 2003, the extensive observations of the Galactic plane by Cerenkov telescopes have detected more than 80 Galactic $\mathrm{TeV}$ sources (Hinton \& Hofmann 2009). Pulsar wind nebulae (PWNe) are the dominant class with more than 30 firm identifications. A similar number of Galactic sources cannot be associated with a counterpart at any other wavelength; they form the unidentified (UNID) source class. The third largest class of Galactic sources are the supernova remnants (SNRs).

The Large Area Telescope (LAT) on board the Fermi Gammaray Space Telescope provides all-sky coverage of the $\gamma$-ray sky at energies from $20 \mathrm{MeV}$ to more than $300 \mathrm{GeV}$. With 2 yr of observations, the Fermi-LAT Second Source Catalog (2FGL; Nolan et al. 2012) reports the detection of 1873 sources, 1298 being identified and 575 without clear identification. Four hundred of them lie within $5^{\circ}$ of the Galactic plane.

Most of the LAT UNID sources are expected to be pulsars, SNRs, binary systems, or PWNe. The $\gamma$-ray emission from these sources is expected to be either hadronic or leptonic. In the leptonic scenario, $\gamma$-ray photons are created by inverse Compton

\footnotetext{
58 Resident at Naval Research Laboratory, Washington, DC 20375, USA.

${ }^{59}$ Royal Swedish Academy of Sciences Research Fellow, funded by a grant from the K. A. Wallenberg Foundation.

${ }^{60}$ Funded by contract ERC-StG-259391 from the European Community.

${ }^{61}$ Funded by a Marie Curie IOF, FP7/2007-2013, grant agreement No. 275861 .

62 NASA Postdoctoral Program Fellow, USA.
}

(IC) scattering of highly relativistic leptons from the source on the ambient photon fields such as cosmic microwave background (CMB), stellar radiation, or infrared emission from dust (e.g., de Jager et al. 2009; de Jager \& Djannati-Ataï 2009). This leptonic approach could well explain several UNIDs (e.g., Tibolla 2011; Tibolla et al. 2012); moreover, one of its biggest advantages is that it provides a natural explanation for the UNIDs that lack a lower energy (radio and X-ray) counterpart (e.g., de Jager et al. 2009; H.E.S.S. Collaboration et al. 2012b), i.e., the so-called dark sources. In the hadronic scenario, hadrons accelerated by a source collide with the nuclei in the ambient medium (e.g., molecular cloud) and secondary neutral pions decay to $\gamma$-rays (e.g., Gabici et al. 2009).

The leptonic PWN scenario requires an energetic and young pulsar to be present. Pulsars are the largest class of Galactic sources detected above $100 \mathrm{MeV}$ with the LAT. These pulsars could make up part of the LAT UNID population. In the LAT energy range, pulsars are point-like sources and exhibit powerlaw spectra with exponential cutoffs between 0.5 and $6 \mathrm{GeV}$ (Abdo et al. 2010e), while PWNe have hard power-law spectra without cutoffs in the $\mathrm{GeV}$ energy range and might be spatially resolved by the LAT. Middle-aged SNRs, interacting with molecular clouds, detected by the LAT are generally bright and exhibit a break at $\sim 2 \mathrm{GeV}$ (Uchiyama 2011). Radio, X-ray, and $\gamma$-ray photons probe the non-thermal particle populations and therefore provide information to discriminate between scenarios in which the $\gamma$-ray emission is dominated by leptonic or hadronic processes. 
Here we report on the analysis of 58 PWNe and Galactic UNIDs detected at $\mathrm{TeV}$ energies, using 45 months of Fermi-LAT data above $10 \mathrm{GeV}$. A complementary search for PWNe in the off-peak phase ranges of pulsars for which the LAT sees $\gamma$ ray pulsations (henceforth "LAT-detected pulsars"), updating the analysis of Ackermann et al. (2011), is presented in the second Fermi-LAT catalog of $\gamma$-ray pulsars (Abdo et al. 2013), henceforth "2PC."

The objective of this work is to constrain some general characteristics of PWNe such as their $\gamma$-ray efficiency. This study of $\mathrm{TeV}$ sources might also increase the number of PWN candidates detected at $\mathrm{GeV}$ energies by the LAT. Slane et al. (2010), Grondin et al. (2011), and Rousseau et al. (2012) have demonstrated the potential of LAT observations to study PWN candidates. With the exception of Vela X (Abdo et al. 2010c), the five PWNe firmly identified by Fermi are associated with TeV counterparts. Furthermore, their spectra are consistent with predictions from a leptonic PWN scenario where the IC spectra peak above $100 \mathrm{GeV}$ (Abdo et al. 2010b; Grondin et al. 2011).

In Section 2, we establish a list of $\mathrm{TeV}$ sources potentially associated with PWNe. In Section 3, we describe the methods and tools we used to analyze LAT data. In Section 4, we describe the spectral and spatial analysis of the LAT data, and in Section 5, we perform a population study based on these new $\gamma$-ray results.

\section{TeV $\gamma$-RAY SOURCE SAMPLE}

We selected our PWN candidates from the online catalog of TeV $\gamma$-ray sources, TeVCat. ${ }^{63}$ As of 2013 January 1, the catalog contained 143 sources observed with the very high energy (VHE) experiments: H.E.S.S. (Aharonian et al. 2006c), VERITAS (Weekes et al. 2002), MAGIC (Aleksić et al. 2012), Milagro (Atkins et al. 2003), and others.

Except for N157 B (Komin et al. 2012), all PWNe detected by VHE experiments lie inside our Galaxy. A survey of the Galactic plane was performed by H.E.S.S., and the current version (Gast et al. 2012) covers $\pm 4^{\circ}$ in latitude and longitudes between $l=65^{\circ}$ and $l=250^{\circ}$. Another survey, specific to the Cygnus region, was performed by VERITAS (Weinstein 2009). Milagro surveyed the northern hemisphere, covering the Galactic plane from $l=30^{\circ}$ to $l=220^{\circ}$ (Abdo et al. 2007). To be conservative compared to the H.E.S.S. survey, we selected the 84 sources that lie within $5^{\circ}$ of the Galactic plane. The Galactic center is a complex region to investigate with FermiLAT, due to the confusion by the large density of sources and by the diffuse emission, so we removed the three VHE sources within $2^{\circ}$ of the Galactic center from our list. These sources, HESS J1745-303 (Aharonian et al. 2008b), HESS J1741-302 (Tibolla et al. 2008), and SNR G0.9+0.1 (Aharonian et al. 2005d), will be presented separately.

We also excluded from our list the $21 \mathrm{TeV} \gamma$-ray sources associated with radio-detected SNRs. LAT observations of these objects will be presented in the Fermi-LAT catalog of SNRs, henceforth "SNR catalog." Finally, we excluded the Crab Nebula and Vela X, both already studied in detail (Abdo et al. 2010b; Buehler et al. 2012; Grondin et al. 2013). The final list of $58 \mathrm{TeV}$ $\gamma$-ray sources that we selected is presented in Table 1 along with their best-fit morphologies measured by VHE experiments.

\footnotetext{
${ }^{63}$ TeVCat is developed by the University of Chicago and can be obtained
} from http://tevcat.uchicago.edu.

\section{CONVENTIONS AND METHODS}

The LAT is a $\gamma$-ray telescope that detects photons by conversion into electron-positron pairs. It operates in the energy range between $20 \mathrm{MeV}$ and more than $300 \mathrm{GeV}$. Details of the instrument and data processing are given in Atwood et al. (2009). The on-orbit calibration is described in Abdo et al. (2009e) and Ackermann et al. (2012). This section will present the data set and the method used to analyze LAT data.

\subsection{Data Set}

This paper uses 45 months of data collected from 2008 August 4 to 2012 April 18 (mission elapsed time: $239,557,440-356,439,741$ s) for regions centered on the positions of each VHE source. We excluded $\gamma$-rays coming from a zenith angle larger than $100^{\circ}$. We used the Pass 7 Clean event class that has substantially less instrumental background above $10 \mathrm{GeV}$ with only a marginal loss in effective area, compared to the Pass 7 Source event class (Ackermann et al. 2012).

We analyzed LAT data only between 10 and $316 \mathrm{GeV}$ to avoid systematics associated with the modeling of adjacent sources with soft spectra. It also reduces systematics associated with imperfect modeling of the Galactic diffuse emission. The maximum energy of $316 \mathrm{GeV}$ increases the overlap between the energy range covered by the VHE experiments and the LAT. The 100-316 GeV energy range was also shown to be crucial in previous analyses like Rousseau et al. (2012).

Figure 1 shows a background-subtracted count map of the Galactic plane observed by the LAT above $10 \mathrm{GeV}$. The bright Vela $(l, b=263.55,-2.79)$ and Geminga $(l, b=195.13,4.27)$ pulsars and the SNR IC $443(l, b=189.06,3.23)$ clearly stand out. In addition to these well-known objects, a large number of other sources are apparent. Several are coincident with sources detected by VHE experiments, such as HESS J1614-518 and HESS J1616-508 (Lande et al. 2012), and will be discussed in Section 4. The large number of other sources visible in the map highlights the LAT sensitivity at high energies.

\subsection{Modeling the Regions of Interest}

Two different software packages for maximum likelihood fitting were used to analyze LAT data: gtlike and pointlike. These tools fit LAT data with a parameterized model of the sky, including models for the instrumental, extragalactic, and Galactic components of the background.

The first one, gtlike, is a maximum likelihood method distributed in the Fermi Science Tools by the FSSC. ${ }^{64} \mathrm{We}$ used version 09-28-00 of the package in binned mode. The second, pointlike, is an alternate software package that we used to fit the positions of point-like sources and fit the spatial parameters of spatially extended sources. Kerr (2011) describes the implementation of pointlike, and Lande et al. (2012) validate pointlike's extension-fitting functionality. In pointlike, the data are binned spatially, using a HEALPix pixelization $^{65}$ (Górski et al. 2005), and spectrally, and the likelihood is maximized over all bins in a region.

We used pointlike to evaluate a position and extension estimate in the LAT data for each source of our sample. Using those morphologies, we used gtlike to obtain the best-fit spectral parameters and statistical significances. gtlike makes fewer approximations in calculating the likelihood for spectra

\footnotetext{
${ }_{64}$ Fermi Science Support Center: http://fermi.gsfc.nasa.gov/ssc/.

65 The HEALPix libraries can be obtained from http://healpix.jpl.nasa.gov/.
} 
Table 1

List of Analyzed VHE Sources

\begin{tabular}{|c|c|c|c|c|c|c|c|}
\hline Name & Class & $\begin{array}{c}l \\
(\mathrm{deg})\end{array}$ & $\begin{array}{c}b \\
\text { (deg) }\end{array}$ & TeV Morphology & $\begin{array}{c}\sigma_{1} \\
(\operatorname{deg})\end{array}$ & $\begin{array}{c}\sigma_{2} \\
(\mathrm{deg})\end{array}$ & Reference \\
\hline VER J0006 + 727 & PWN & 119.58 & 10.20 & PS & $\cdots$ & ... & Aliu et al. (2013) \\
\hline MGRO J0631 + 105 & PWN & 201.30 & 0.51 & PS & $\cdots$ & $\cdots$ & Abdo et al. (2009f) \\
\hline MGRO J0632 + 17 & PWN & 195.34 & 3.78 & $\mathrm{G}$ & 1.30 & $\cdots$ & Abdo et al. (2009f) \\
\hline HESS J1018-589 & UNID & 284.23 & -1.72 & PS & $\ldots$ & $\ldots$ & H.E.S.S. Collaboration et al. (2012a) \\
\hline HESS J1023-575 & MSC & 284.22 & -0.40 & G & 0.18 & $\cdots$ & H.E.S.S. Collaboration et al. (2011d) \\
\hline HESS J1026-582 & PWN & 284.80 & -0.52 & $\mathrm{G}$ & 0.14 & $\cdots$ & H.E.S.S. Collaboration et al. (2011d) \\
\hline HESS J1119-614 & PWN & 292.10 & -0.49 & G & 0.05 & $\ldots$ & Presentation $^{\mathrm{a}}$ \\
\hline HESS J1303-631 & PWN & 304.24 & -0.36 & $\mathrm{G}$ & 0.16 & $\cdots$ & Aharonian et al. (2005c) \\
\hline HESS J1356-645 & PWN & 309.81 & -2.49 & G & 0.20 & $\cdots$ & H.E.S.S. Collaboration et al. (2011c) \\
\hline HESS J1418-609 & PWN & 313.25 & 0.15 & EG & 0.08 & 0.06 & Aharonian et al. (2006a) \\
\hline HESS J1420-607 & PWN & 313.56 & 0.27 & $\mathrm{G}$ & 0.06 & $\cdots$ & Aharonian et al. (2006a) \\
\hline HESS J1427-608 & UNID & 314.41 & -0.14 & EG & 0.04 & 0.08 & Aharonian et al. (2008c) \\
\hline HESS J1458-608 & PWN & 317.75 & -1.70 & $\mathrm{G}$ & 0.17 & $\cdots$ & de los Reyes et al. (2012) \\
\hline HESS J1503-582 & UNID & 319.62 & 0.29 & G & 0.26 & $\cdots$ & Renaud et al. (2008) \\
\hline HESS J1507-622 & UNID & 317.95 & -3.49 & $\mathrm{G}$ & 0.15 & $\cdots$ & H.E.S.S. Collaboration et al. (2011e) \\
\hline HESS J1514-591 & PWN & 320.33 & -1.19 & EG & 0.11 & 0.04 & Aharonian et al. (2005b) \\
\hline HESS J1554-550 & PWN & 327.16 & -1.07 & PS & $\ldots$ & $\ldots$ & Acero et al. (2012) \\
\hline HESS J1614-518 & MSC & 331.52 & -0.58 & EG & 0.23 & 0.15 & Aharonian et al. (2006d) \\
\hline HESS J1616-508 & PWN & 332.39 & -0.14 & $\mathrm{G}$ & 0.14 & $\cdots$ & Aharonian et al. (2006d) \\
\hline HESS J1626-490 & UNID & 334.77 & 0.05 & EG & 0.07 & 0.10 & Aharonian et al. (2008c) \\
\hline HESS J1632-478 & PWN & 336.38 & 0.19 & EG & 0.21 & 0.06 & Aharonian et al. (2006d) \\
\hline HESS J1634-472 & UNID & 337.11 & 0.22 & $\mathrm{G}$ & 0.11 & $\cdots$ & Aharonian et al. (2006d) \\
\hline HESS J1640-465 & PWN & 338.32 & -0.02 & $\mathrm{G}$ & 0.04 & $\cdots$ & Aharonian et al. (2006d) \\
\hline HESS J1646-458A & MSC & 339.57 & -0.02 & $\mathrm{G}$ & 0.35 & $\ldots$ & Abramowski et al. (2012) \\
\hline HESS J1646-458B & MSC & 339.01 & -0.79 & $\mathrm{G}$ & 0.25 & $\cdots$ & Abramowski et al. (2012) \\
\hline HESS J1702-420 & UNID & 344.30 & -0.18 & EG & 0.30 & 0.15 & Aharonian et al. (2006d) \\
\hline HESS J1708-443 & PWN & 343.06 & -2.38 & $\mathrm{G}$ & 0.29 & $\ldots$ & H.E.S.S. Collaboration et al. (2011b) \\
\hline HESS J1718-385 & PWN & 348.83 & -0.49 & EG & 0.15 & 0.07 & Aharonian et al. (2007) \\
\hline HESS J1729-345 & UNID & 353.44 & -0.13 & $\mathrm{G}$ & 0.14 & $\ldots$ & H.E.S.S. Collaboration et al. (2011a) \\
\hline HESS J1804-216 & UNID & 8.40 & -0.03 & EG & 0.16 & 0.27 & Aharonian et al. (2006d) \\
\hline HESS J1809-193 & PWN & 11.18 & -0.09 & EG & 0.53 & 0.25 & Aharonian et al. (2007) \\
\hline HESS J1813-178 & PWN & 12.81 & -0.03 & $\mathrm{G}$ & 0.04 & $\ldots$ & Aharonian et al. (2006d) \\
\hline HESS J1818-154 & PWN & 15.41 & 0.17 & $\mathrm{G}$ & 0.14 & $\cdots$ & Hofverberg et al. (2011) \\
\hline HESS J1825-137 & PWN & 17.71 & -0.70 & EG & 0.13 & 0.12 & Aharonian et al. (2006b) \\
\hline HESS J1831-098 & PWN & 21.85 & -0.11 & $\mathrm{G}$ & 0.15 & $\ldots$ & Sheidaei (2011) \\
\hline HESS J1833-105 & PWN & 21.51 & -0.88 & PS & $\ldots$ & $\ldots$ & Djannati-Ată et al. (2008) \\
\hline HESS J1834-087 & UNID & 23.24 & -0.31 & $\mathrm{G}$ & 0.09 & $\ldots$ & Aharonian et al. (2006d) \\
\hline HESS J1837-069 & UNID & 25.18 & -0.12 & EG & 0.12 & 0.05 & Aharonian et al. (2006d) \\
\hline HESS J1841-055 & UNID & 26.80 & -0.20 & EG & 0.41 & 0.25 & Aharonian et al. (2008c) \\
\hline HESS J1843-033 & UNID & 29.30 & 0.51 & PS & $\ldots$ & $\ldots$ & Hoppe (2008) \\
\hline MGRO J1844-035 & UNID & 28.91 & -0.02 & PS & $\cdots$ & $\ldots$ & Abdo et al. (2009f) \\
\hline HESS J1846-029 & PWN & 29.70 & -0.24 & PS & $\ldots$ & $\ldots$ & Djannati-Ată et al. (2008) \\
\hline HESS J1848-018 & UNID & 31.00 & -0.16 & G & 0.32 & $\ldots$ & Chaves et al. (2008) \\
\hline HESS J1849-000 & PWN & 32.64 & 0.53 & PS & $\ldots$ & $\ldots$ & Terrier et al. (2008) \\
\hline HESS J1857 + 026 & UNID & 35.96 & -0.06 & EG & 0.11 & 0.08 & Aharonian et al. (2008c) \\
\hline HESS J1858 + 020 & UNID & 35.58 & -0.58 & EG & 0.08 & 0.02 & Aharonian et al. (2008c) \\
\hline MGRO J1900 + 039 & UNID & 37.42 & -0.11 & PS & $\cdots$ & $\cdots$ & Abdo et al. (2009f) \\
\hline MGRO J1908 + 06 & UNID & 40.39 & -0.79 & G & 0.34 & $\cdots$ & Aharonian et al. (2009) \\
\hline HESS J1912 + 101 & PWN & 44.39 & -0.07 & $\mathrm{G}$ & 0.26 & $\cdots$ & Aharonian et al. (2008a) \\
\hline VER J1930 + 188 & PWN & 54.10 & 0.26 & PS & $\cdots$ & $\cdots$ & Acciari et al. (2010) \\
\hline MGRO J1958 + 2848 & PWN & 65.85 & -0.23 & PS & $\cdots$ & $\cdots$ & Abdo et al. (2009f) \\
\hline VER J1959 + 208 & PSR & 59.20 & -4.70 & PS & $\cdots$ & $\cdots$ & Hall et al. (2003) \\
\hline VER J2016 + 372 & UNID & 74.94 & 1.15 & PS & $\cdots$ & $\cdots$ & Aliu (2011) \\
\hline MGRO J2019 + 37 & PWN & 75.00 & 0.39 & G & 0.55 & $\cdots$ & Abdo et al. (2007) \\
\hline MGRO J2031 + 41A & UNID & 79.53 & 0.64 & G & 1.50 & $\cdots$ & Abdo et al. (2007) \\
\hline MGRO J2031 + 41B & UNID & 80.25 & 1.07 & $\mathrm{G}$ & 0.10 & $\cdots$ & Bartoli et al. (2012) \\
\hline MGRO J2228 + 61 & PWN & 106.57 & 2.91 & PS & $\ldots$ & $\cdots$ & Abdo et al. (2009f) \\
\hline W49A & SFR & 43.27 & -0.00 & PS & $\cdots$ & $\cdots$ & Brun et al. (2011) \\
\hline
\end{tabular}

Notes. VHE sources analyzed with LAT observations. The first two columns list the VHE source names and classifications as defined in the TeV catalog (see Section 2): PWN for pulsar wind nebulae, PSR for pulsars, UNID for unidentified sources, MSC for massive star clusters, and SFR for star-forming regions. The third and fourth columns give the Galactic longitude and latitude for each source. The fifth column presents the best-fit morphology of the source when observed by VHE experiments: PS, G, and EG, respectively, stand for point source, Gaussian, and elliptical Gaussian. The sixth and seventh columns present the Gaussian and elliptical Gaussian extensions. A reference is cited in the eighth column.

a This work was presented at the "Supernova Remnants and Pulsar Wind Nebulae in the Chandra Era," 2009. See http://cxc.harvard.edu/cdo/snr09/pres/DjannatiAtai_Arache_v2.pdf. 

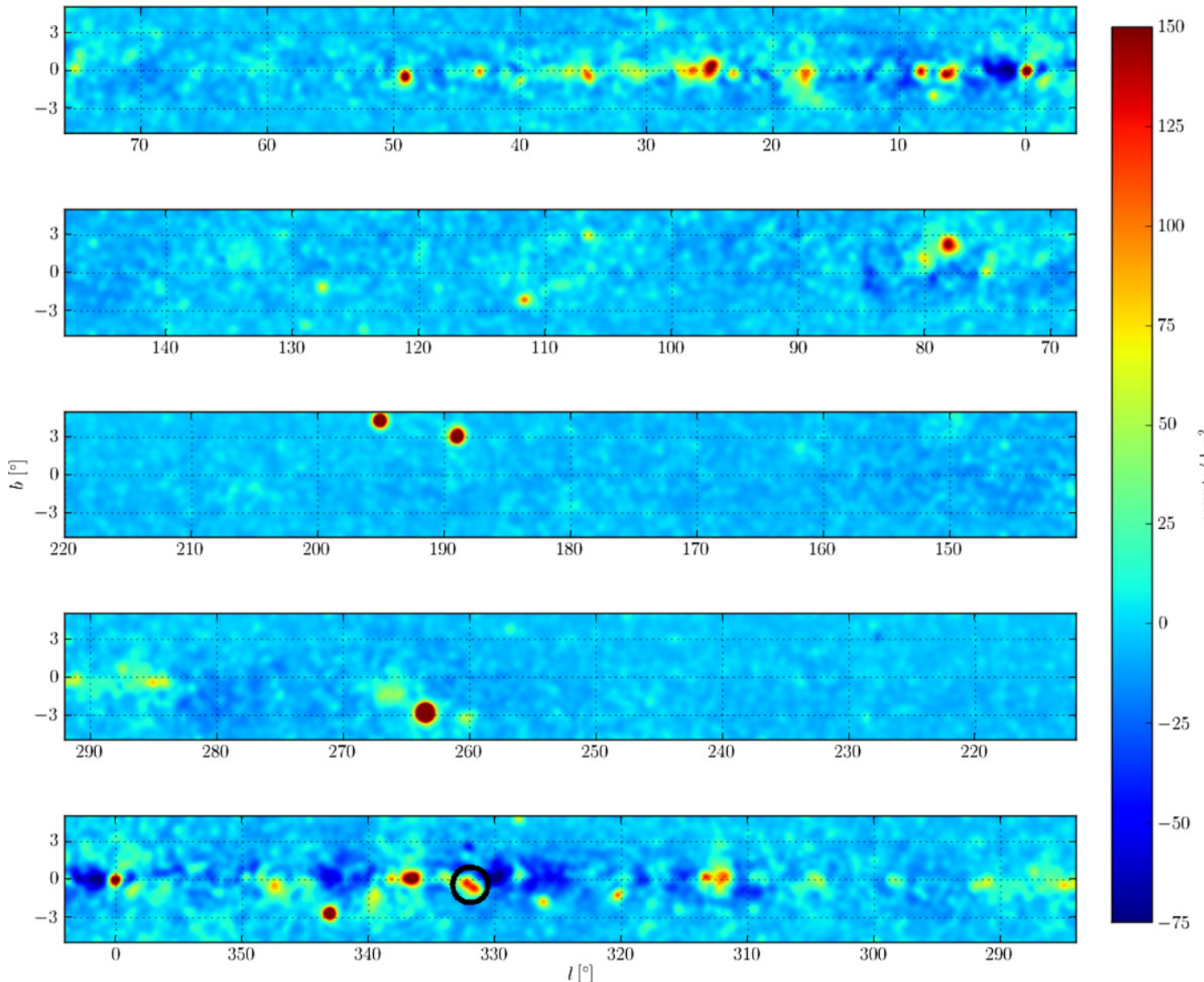

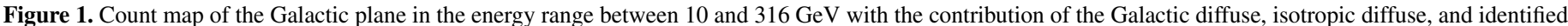

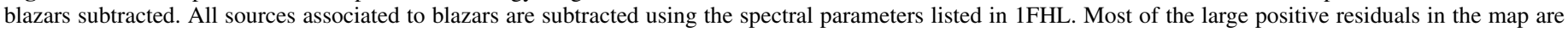

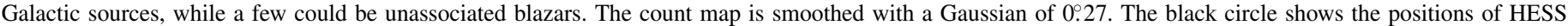
J1614-518 and HESS J1616-508.

(A color version of this figure is available in the online journal.)

than pointlike. Both methods agree with each other within $10 \%$ for all derived quantities, but all spectral parameters quoted in the following were obtained using gtlike.

Since pointlike and gtlike use two different shapes for the regions of the sky modeled for each source, we included photons within a radius of $5^{\circ}$ centered on our source of interest when using pointlike and within a $7^{\circ} \times 7^{\circ}$ square region aligned with Galactic coordinates when using gtlike. We tried to keep the two methods as close as possible by using the same conventions, i.e., same energy binning of eight energy bins per decade between 10 and $316 \mathrm{GeV}$, and the same optimizer: MINUIT (James \& Roos 1975).

The Galactic diffuse emission was modeled by the standard LAT interstellar emission model ring_2yearp7v6_v0.fits. The residual cosmic-ray background and extragalactic radiation are described by a single isotropic component with a spectral shape described by the file isotrop_2year_P76_clean_ v0.txt. The models have been released and described by the Fermi-LAT Collaboration through the FSSC. ${ }^{66}$ In the following, we fit the Galactic diffuse normalization. Since the isotropic diffuse component is not well constrained over the small regions of interest used in this work, we fixed its normalization to 1.

${ }^{66}$ Background models are available at http://fermi.gsfc.nasa.gov/ssc/data/access/lat/BackgroundModels.html.
We included in our sky model all cataloged LAT sources within a radius of $10^{\circ}$ of each source of interest and listed in the hard source list (Paneque et al. 2013), henceforth "1FHL." The 1FHL catalog is a forthcoming catalog of sources using $3 \mathrm{yr}$ of LAT data above $10 \mathrm{GeV}$. The data selection used clean events as done in this work. The spectral parameters of sources closer than $2^{\circ}$ to the source of interest were fit, while the spectra of all other 1FHL sources were fixed.

$\gamma$-ray pulsars are found near many of the VHE sources. Table 2 summarizes the sources analyzed in this paper that are near an LAT-detected pulsar. In the LAT energy range, pulsars tend to be brighter than their associated PWNe (Ackermann et al. 2011). During the fit, the observed photon sample is shared by all modeled sources. The high energy range used in this work prevents a reasonable fit of a pulsar component modeled by a power law with an exponential cutoff spectrum. If a pulsar's model overestimates its emission above $10 \mathrm{GeV}$, a putative PWN emission would be underestimated. This effect could obscure the detection of a faint PWN. On the contrary, not including an existing source, such as a pulsar, would artificially increase the flux attributed to a putative PWN at low energy. Therefore, we decided to keep as separate sources in the model all pulsars located outside the VHE experiment template and more than 0.27 away from the source of interest. The 0.27 radius corresponds to the $68 \%$ containment radius of the 
Table 2

VHE Sources with an LAT-detected Pulsar within 0.5

\begin{tabular}{llllll}
\hline \hline Name & Pulsar Name & $\begin{array}{c}\text { Distance } \\
(\mathrm{deg})\end{array}$ & 2FGL Name & In the Model & Justification \\
\hline VER J0006+727 & PSR J0007+7303 & 0.26 & 2FGL J0007.0+7303 & $\mathrm{N}$ & $\mathrm{a}$ \\
MGRO J0631+105 & PSR J0631+1036 & 0.10 & 2FGL J0631.5+1035 & $\mathrm{N}$ & $\mathrm{a}$ \\
MGRO J0632+17 & PSR J0633+1746 & 0.00 & 2FGL J0633.9+1746 & $\mathrm{N}$ & $\mathrm{a}$ \\
HESS J1018-589 & PSR J1016-5857 & 0.22 & 2FGL J1016.5-5858 & $\mathrm{N}$ & $\mathrm{a}$ \\
HESS J1023-575 & PSR J1023-5746 & 0.05 & 2FGL J1022.7-5741 & $\mathrm{N}$ & $\mathrm{b}$ \\
HESS J1026-582 & PSR J1028-5819 & 0.27 & 2FGL J1028.5-5819 & $\mathrm{Y}$ & $\ldots$ \\
HESS J1119-614 & PSR J1119-6127 & 0.07 & 2FGL J1118.8-6128 & $\mathrm{N}$ & $\mathrm{a}$ \\
HESS J1356-645 & PSR J1357-6429 & 0.12 & 2FGL J1356.0-6436 & $\mathrm{N}$ & $\mathrm{a}$ \\
HESS J1418-609 & PSR J1418-6058 & 0.05 & 2FGL J1418.7-6058 & $\mathrm{N}$ & $\mathrm{b}$ \\
HESS J1420-607 & PSR J1420-6048 & 0.05 & 2FGL J1420.1-6047 & $\mathrm{N}$ & $\mathrm{b}$ \\
HESS J1458-608 & PSR J1459-6053 & 0.17 & 2FGL J1459.4-6054 & $\mathrm{N}$ & $\mathrm{a}$ \\
HESS J1514-591 & PSR J1513-5908 & 0.03 & & $\mathrm{~N}$ & $\mathrm{~b}$ \\
HESS J1646-458B & PSR J1648-4611 & 0.42 & 2FGL J1646-4611 & $\mathrm{N}$ & $\mathrm{c}$ \\
HESS J1702-420 & PSR J1702-4128 & 0.53 & & $\mathrm{~N}$ & $\mathrm{c}$ \\
HESS J1708-443 & PSR J1709-4429 & 0.25 & 2FGL J1709.7-4429 & $\mathrm{N}$ & $\mathrm{b}$ \\
HESS J1718-385 & PSR J1718-3825 & 0.13 & 2FGL J1718.3-3827 & $\mathrm{N}$ & $\mathrm{a}$ \\
HESS J1804-216 & PSR J1803-2149 & 0.27 & 2FGL J1803.3-2148 & $\mathrm{N}$ & $\mathrm{b}$ \\
HESS J1833-105 & PSR J1833-1034 & 0.01 & 2FGL J1833.6-1032 & $\mathrm{N}$ & $\mathrm{a}$ \\
HESS J1841-055 & PSR J1838-0537 & 0.48 & 2FGL J1839.0-0539 & $\mathrm{Y}$ & $\ldots$ \\
MGRO J1908+06 & PSR J1907+0602 & 0.23 & 2FGL J1907.9+0602 & $\mathrm{N}$ & $\mathrm{a}$ \\
MGRO J1958+2848 & PSR J1958+2846 & 0.12 & 2FGL J1958.6+2845 & $\mathrm{N}$ & $\mathrm{N}$ \\
VER J1959+208 & PSR J1959+2048 & 0.02 & 2FGL J1959.5+2047 & $\mathrm{N}$ & $\mathrm{a}$ \\
MGRO J2019+37 & PSR J2021+3651 & 0.36 & 2FGL J2021.0+3651 & $\mathrm{N}$ & $\mathrm{N}$ \\
MGRO J2031+41B & PSR J2032+4127 & 0.05 & 2FGL J2032.2+4126 & $\mathrm{N}$ & $\mathrm{b}$ \\
MGRO J2228+61 & PSR J2229+6114 & 0.09 & 2FGL J2229+6114 & $\mathrm{N}$ & \\
\hline
\end{tabular}

Notes. Sources with an LAT-detected $\gamma$-ray pulsar within 0.5 . The first two columns list the names of the VHE sources and their associated pulsars. The third column is the angular distance between the center of the VHE source and the LAT pulsar. The pulsar position comes from 2PC. The fourth column gives the pulsar 2FGL name. The fifth column says if the emission from the pulsar was included in the model of the background emission. " $\mathrm{Y}$ " means the emission was included and " $\mathrm{N}$ " means that it was not. The sixth column gives the justification when the pulsar is not included in the model: a-the distance between the pulsar and the source is closer than 0.27 ; $b$ - the pulsar is located inside the edge of the shape observed by VHE experiments; $c$ - not "a" and not "b," but no significant excess above $10 \mathrm{GeV}$ at the position of the pulsar.

point-spread function (PSF) averaged over energies above $10 \mathrm{GeV}$ (Ackermann et al. 2012). For VHE sources less than 0.27 from an LAT-detected pulsar, we analyzed the sources twice. Removing the pulsar from the background model amounts to neglecting it, while leaving it in the background model amounts to subtracting the underlying pulsar contribution from each putative PWN. In all cases, the parameters of the pulsar models have been fixed to those obtained in the 2FGL catalog (Nolan et al. 2012). If contamination from the pulsar is a concern, it is possible to phase-fold photons and analyze the data in the off-peak phase intervals of the pulsar. This will be performed in $2 \mathrm{PC}$. Here we analyze data at all phases to have the largest possible statistics and therefore better sensitivity to faint PWNe.

Due to the 45 month integration time of our analysis compared to the 36 months of the forthcoming 1FHL catalog, we expected to find new statistically significant background sources. To prevent bias from these sources, we included in our model any nearby background sources with a significance above $4 \sigma$ (TS > 25 with 4 degrees of freedom, dof). We fit their spectra with power laws. The locations and spectra of the six such sources found in our analysis are described in Table 3.

\subsection{Analysis Procedure}

We developed a uniform procedure for analyzing any potential emission in the 58 regions listed in Table 1 . The small statistics above $10 \mathrm{GeV}$ and the narrow energy range (1.5 decade) prevent any spectral curvature from being significant as will be discussed in the 1FHL catalog. Therefore, in the following we derived the spectra assuming a power-law spectral model:

$$
\frac{d F}{d E}=N_{0}\left(\frac{E}{E_{0}}\right)^{-\Gamma},
$$

where $N_{0}$ is the normalization, $\Gamma$ is the spectral index, and $E_{0}$ is the scale parameter. To minimize the covariance between $N_{0}$ and $\Gamma$, we ran the analysis twice. In the first iteration, we fitted the source assuming a power-law model depending on the integral flux $F$ and $\Gamma$,

$$
\frac{d F}{d E}=\frac{F(-\Gamma+1) E^{-\Gamma}}{E_{\max }^{-\Gamma+1}-E_{\min }^{-\Gamma+1}} .
$$

Using the covariance matrix of the fit parameters, we derived the pivot energy $E_{p}$, computed as the energy at which the relative uncertainty on the normalization $N_{0}$ was minimal (Nolan et al. 2012). In a second iteration, we refitted the spectrum of the source assuming a power-law spectral model (Equation (1)) with the scale parameter $E_{0}$ fixed to $E_{p}$. For sources not significantly detected, we computed a $99 \%$ confidence level (c.l.) Bayesian upper limit on the flux of the source assuming the published VHE morphology and a power-law photon spectral index of 2 .

We performed the spatial analysis in two steps. As a first step, we assumed that the LAT emission originates from the same population of emitting particles cooling by the same radiation process as the VHE emission. These assumptions mean that 
Table 3

Additional Background Sources

\begin{tabular}{lccccc}
\hline \hline Name & $\begin{array}{c}l \\
(\mathrm{deg})\end{array}$ & $\begin{array}{c}b \\
(\mathrm{deg})\end{array}$ & TS & $\begin{array}{c}\text { Prefactor } \\
\left(\mathrm{cm}^{-2} \mathrm{~s}^{-1} \mathrm{MeV}^{-1}\right)\end{array}$ & $\begin{array}{c}\text { Spectral Index } \\
\text { 2FGL J1405.5-6121 }\end{array}$ \\
Background Source 1 & 311.81 & 0.30 & 31 & $(1.2 \pm 0.4) \times 10^{-15}$ & $1.8 \pm 0.3$ \\
Background Source 2 & 333.59 & -0.31 & 29 & $(6.5 \pm 2.5) \times 10^{-17}$ & $4.3 \pm 0.9$ \\
2FGL J1823.1-1338c & 336.96 & -0.07 & 25 & $(1.2 \pm 0.4) \times 10^{-15}$ & $1.9 \pm 0.4$ \\
2FGL J1836.8-0623c & 17.51 & -0.12 & 30 & $(4.9 \pm 1.9) \times 10^{-15}$ & $2.9 \pm 0.7$ \\
PSR J1838-0536 & 25.41 & 0.42 & 25 & $(9.4 \pm 3.9) \times 10^{-16}$ & $2.0 \pm 0.4$ \\
\hline
\end{tabular}

Notes. New background sources found in our analysis for energies above $10 \mathrm{GeV}$ that are not included in $1 \mathrm{FHL}$. The first three columns are their names, Galactic longitudes, and Galactic latitudes. The TS values for the sources are provided in the fourth column. The spectral results are presented in the fifth and sixth columns assuming a power-law spectral model (Equation (1)) with a scale parameter $E_{0}=56.2 \mathrm{GeV}$ (corresponding to the mid-value of the energy range in logarithmic scale). PSR J1838-0536 improves the morphology fit of the diffuse source HESS J1841-055. The spectral fit is consistent with the pulsar component (spectral index of $\sim 4$ ). Additional sources appearing to be spatially consistent with a 2 FGL source are labeled with the name of the associated 2FGL source.

there should be a correlation between the spatial morphology of a source at LAT and VHE energies. While it is possible for the $\gamma$-ray emission from PWNe to have both the synchrotron and IC components visible in the LAT energy range (as in the case of the Crab Nebula; Abdo et al. 2010b), this scenario is unlikely for observations above $10 \mathrm{GeV}$ since electrons of energy around $1 \mathrm{PeV}$ should radiate in a magnetic field $\sim 0.3 \mathrm{G}$ to emit photons above $10 \mathrm{GeV}$. This value is unrealistic for a PWN. A second exception would appear if the LAT and VHE emissions originate from two different populations of electrons (as in the case of Vela X; Abdo et al. 2010c). However, most PWNe observed by the LAT show only IC emission from the same population responsible for the VHE emission (e.g., Abdo et al. 2010a; Grondin et al. 2011; Rousseau et al. 2012), supporting our assumption in this first step.

We assumed that any LAT emission would have the same morphology as the best-fit VHE morphology. Therefore, we modeled the spatial distribution of the emission as Gaussian with a position and extension fixed at the value of the spatial best fit performed in the VHE energy range. To homogenize the analysis, if the source was modeled with an elliptical Gaussian at VHE, the Gaussian was fixed to the averaged extension. We tested for the significance of the source at LAT energies using a likelihood-ratio test: TS $=2 \times \log \left(\mathcal{L}_{1} / \mathcal{L}_{0}\right)$, where $\mathcal{L}$ is the Poisson likelihood of obtaining the observed data given the assumed model, $\mathcal{L}_{1}$ corresponds to the likelihood obtained by fitting a model of the source of interest and the background model, and $\mathcal{L}_{0}$ corresponds to the likelihood obtained by fitting the background model only. In the following, we refer to the TS assuming the VHE shape as $\mathrm{TS}_{\mathrm{TeV}}$. By assuming a fixed spatial model, our test has fewer dof, which makes our test more sensitive to the LAT emission, assuming that the VHE spatial model reproduces the LAT observation.

The formal statistical significance of this test can be obtained from the Wilks theorem (Wilks 1938). In the null hypothesis, TS follows a $\chi^{2}$ distribution with $n$ dof where $n$ is the number of additional parameters in the model. We consider a source to be significantly detected when $\mathrm{TS}_{\mathrm{TeV}} \geqslant 16$. Our test has only 2 dof (the flux and the spectral index), so our threshold corresponds to a formal significance of $3.6 \sigma$. For significantly detected sources, $\mathrm{TS}_{\mathrm{TeV}}$ is presented in Table 4.

As a second step, for significantly detected sources, we then independently characterized the best-fit morphology obtained from the LAT emission. Following the method of Nolan et al. (2012), we assumed the source to be point-like and fitted its position. Following the method adopted in Lande et al. (2012), we then assumed the source to be spatially extended with a Gaussian spatial model and fitted its position and extension. From this, we obtained $\mathrm{TS}_{\text {point }}$ and $\mathrm{TS}_{\text {Gaussian. }}$. We then defined the extension significance $\mathrm{TS}_{\text {ext }}=\mathrm{TS}_{\mathrm{Gaussian}}-\mathrm{TS}_{\text {point }}$ following the method of Lande et al. (2012) and set the threshold for claiming the source to be spatially extended as $\mathrm{TS}_{\text {ext }}>16$, corresponding to a significance of $4 \sigma . \mathrm{TS}_{\mathrm{GeV}}=\mathrm{TS}_{\mathrm{Gaussian}}$ when $\mathrm{TS}_{\text {ext }}>16$ and $\mathrm{TS}_{\mathrm{GeV}}=\mathrm{TS}_{\text {point }}$ otherwise. Table 4 lists 8 significantly extended sources and 22 point sources. If the source was not significantly extended, we derived a $99 \%$ c.l. Bayesian upper limit on the extension.

The LAT spectra of the sources have been derived assuming the published VHE morphology. In addition to performing a spectral fit over the entire energy range, we computed a spectral energy distribution (SED) by fitting the flux of the source independently in three energy bins spaced uniformly in log from $10 \mathrm{GeV}$ to $316 \mathrm{GeV}$. During this fit, we fixed the spectral index of the source at 2 as well as the model of background sources to the best fit obtained in the whole energy range. We define a detection in the energy bin when TS $\geqslant 10$ and otherwise compute a flux upper limit using the same method as for the fit in the full energy range. All spectral results are presented in Tables 5 and 6.

\subsection{Systematics}

Two main systematic uncertainties can affect the extension fit: uncertainties in our model of the Galactic diffuse emission and uncertainties in our knowledge of the LAT PSF. We used the procedure described in Lande et al. (2012) and obtained the total systematic error on the source extension by adding the two errors in quadrature.

Three main systematic uncertainties can affect the LAT flux estimate for an extended source: uncertainties on the Galactic diffuse background, on the effective area, and on the shape of the source. We combined these errors in quadrature to obtain an estimate of the total systematic uncertainty on spectral parameters.

The dominant uncertainty comes from the Galactic diffuse emission and was estimated by using the alternative model for Galactic diffuse emission described in Lande et al. (2012). The systematic due to the effective area was determined by using modified instrument response functions as explained in Ackermann et al. (2012). 
Table 4

Spatial Results for Detected Sources

\begin{tabular}{|c|c|c|c|c|c|c|c|c|}
\hline Name & ID & $\mathrm{TS}_{\mathrm{TeV}}$ & $\mathrm{TS}_{\mathrm{GeV}}$ & $\mathrm{TS}_{\mathrm{ext}}$ & $\begin{array}{c}l \\
(\operatorname{deg})\end{array}$ & $\begin{array}{c}b \\
(\operatorname{deg})\end{array}$ & $\begin{array}{l}\mathrm{Unc}_{\mathrm{GeV}} \\
(\mathrm{deg})\end{array}$ & $\sigma$ \\
\hline \multirow{2}{*}{$\begin{array}{l}\text { VER J0006+727 } \\
\text { a }\end{array}$} & \multirow[t]{2}{*}{ PSR } & 655 & 1206 & 0 & 119.68 & 10.47 & $0.01,0.01$ & $<0.07$ \\
\hline & & 2 & $\ldots$ & $\cdots$ & $\ldots$ & $\ldots$ & $\ldots$ & $\ldots$ \\
\hline \multirow{2}{*}{$\begin{array}{l}\text { MGRO J0632+17 } \\
\text { a }\end{array}$} & \multirow[t]{2}{*}{ PSR } & 699 & 2056 & 1 & 195.13 & 4.28 & $0.01,0.01$ & $<0.08$ \\
\hline & & 5 & $\ldots$ & $\cdots$ & $\ldots$ & $\ldots$ & $\ldots$ & $\ldots$ \\
\hline \multirow{2}{*}{$\begin{array}{l}\text { HESS J1018-589 } \\
\text { a }\end{array}$} & \multirow[t]{2}{*}{$\mathrm{O}$} & 29 & 29 & 0 & 284.33 & -1.66 & $0.04,0.02$ & $<0.87$ \\
\hline & & 25 & 25 & 2 & 284.34 & -1.65 & $0.04,0.02$ & $<0.87$ \\
\hline \multirow{2}{*}{$\begin{array}{l}\text { HESS J1023-575 } \\
\text { a }\end{array}$} & \multirow[t]{2}{*}{ PWNc } & 52 & 58 & 8 & 284.13 & -0.45 & $0.03,0.02$ & $<0.77$ \\
\hline & & 52 & 58 & 8 & 284.13 & -0.45 & $0.03,0.02$ & $<0.77$ \\
\hline \multirow{2}{*}{$\begin{array}{l}\text { HESS J1119-614 } \\
\text { a }\end{array}$} & \multirow[t]{2}{*}{ PWNc } & 27 & 27 & 9 & 292.16 & -0.56 & $0.05,0.02$ & $<0.31$ \\
\hline & & 16 & 16 & 9 & 292.18 & -0.57 & $0.05,0.02$ & $<0.32$ \\
\hline HESS J1303-631 & PWNc & 37 & 58 & 29 & 304.56 & -0.11 & $0.04,0.03$ & $0.45 \pm 0.09 \pm 0.10$ \\
\hline \multirow{2}{*}{$\begin{array}{l}\text { HESS J1356-645 } \\
\text { a }\end{array}$} & \multirow[t]{2}{*}{ PWN } & 24 & 26 & 3 & 309.71 & -2.33 & $0.05,0.01$ & $<0.39$ \\
\hline & & 24 & 26 & 3 & 309.71 & -2.32 & $0.05,0.01$ & $<0.39$ \\
\hline \multirow{2}{*}{$\begin{array}{l}\text { HESS J1418-609 } \\
\text { a }\end{array}$} & \multirow[t]{2}{*}{ PSR } & 31 & 32 & 0 & 313.28 & 0.13 & $0.03,0.01$ & $<0.32$ \\
\hline & & 15 & $\cdots$ & $\ldots$ & $\ldots$ & $\ldots$ & $\ldots$ & $\ldots$ \\
\hline \multirow{2}{*}{$\begin{array}{l}\text { HESS J1420-607 } \\
\text { a }\end{array}$} & \multirow[t]{2}{*}{ PWNc } & 42 & 42 & 0 & 313.55 & 0.27 & $0.04,0.02$ & $<0.38$ \\
\hline & & 36 & 36 & 0 & 313.55 & 0.28 & $0.04,0.02$ & $<0.39$ \\
\hline \multirow{2}{*}{$\begin{array}{l}\text { HESS J1507-622 } \\
\text { a }\end{array}$} & \multirow[t]{2}{*}{$\mathrm{O}$} & 21 & 23 & 7 & 317.77 & -3.60 & $0.05,0.03$ & $<1.04$ \\
\hline & & 21 & 23 & 7 & 317.76 & -3.61 & $0.05,0.03$ & $<1.04$ \\
\hline \multirow{2}{*}{$\begin{array}{l}\text { HESS J1514-591 } \\
\text { a }\end{array}$} & \multirow[t]{2}{*}{ PWN } & 156 & 147 & 10 & 320.35 & -1.25 & $0.03,0.01$ & $<0.16$ \\
\hline & & 156 & 147 & 10 & 320.35 & -1.25 & $0.03,0.01$ & $<0.16$ \\
\hline HESS J1614-518 & $\mathrm{O}$ & 110 & 133 & 47 & 331.62 & -0.74 & $0.04,0.03$ & $0.28 \pm 0.03 \pm 0.05$ \\
\hline HESS J1616-508 & PWNc & 75 & 94 & 31 & 332.39 & -0.27 & $0.04,0.02$ & $0.25 \pm 0.03 \pm 0.05$ \\
\hline HESS J1632-478 & PWNc & 137 & 161 & 56 & 336.50 & 0.10 & $0.03,0.02$ & $0.30 \pm 0.06 \pm 0.06$ \\
\hline HESS J1634-472 & $\mathrm{O}$ & 33 & 34 & 1 & 337.23 & 0.35 & $0.03,0.01$ & $<1.21$ \\
\hline HESS J1640-465 & PWNc & 47 & 42 & 9 & 338.33 & 0.05 & $0.05,0.01$ & $<1.17$ \\
\hline \multirow{2}{*}{$\begin{array}{l}\text { HESS J1708-443 } \\
\text { a }\end{array}$} & PSR & 722 & 1153 & 0 & 343.11 & -2.70 & $0.01,0.01$ & $<0.05$ \\
\hline & & 33 & 64 & 0 & 343.12 & -2.70 & $0.01,0.01$ & $<0.09$ \\
\hline HESS J1804-216 & $\mathrm{O}$ & 138 & 141 & 37 & 8.40 & -0.09 & $0.04,0.01$ & $0.25 \pm 0.03 \pm 0.04$ \\
\hline a & & 124 & 128 & 30 & 8.42 & -0.10 & $0.04,0.01$ & $0.24 \pm 0.03 \pm 0.04$ \\
\hline HESS J1825-137 & PWN & 56 & 82 & 30 & 17.55 & -0.47 & $0.05,0.03$ & $0.44 \pm 0.08 \pm 0.09$ \\
\hline HESS J1834-087 & $\mathrm{O}$ & 27 & 36 & 4 & 23.20 & -0.26 & $0.05,0.01$ & $<0.22$ \\
\hline HESS J1837-069 & PWNc & 73 & 119 & 46 & 25.17 & 0.00 & $0.05,0.03$ & $0.36 \pm 0.06 \pm 0.04$ \\
\hline HESS J1841-055 & PWNc & 64 & 70 & 32 & 27.01 & -0.15 & $0.05,0.03$ & $0.38 \pm 0.06 \pm 0.06$ \\
\hline HESS J1848-018 & PWNc & 19 & 19 & 0 & 30.90 & -0.20 & $0.04,0.01$ & $<1.50$ \\
\hline HESS J1857+026 & PWNc & 53 & 55 & 8 & 36.08 & 0.02 & $0.04,0.01$ & $<0.28$ \\
\hline MGRO J1908+06 & PSR & 16 & 37 & 2 & 40.11 & -0.84 & $0.03,0.01$ & $<0.19$ \\
\hline a & & 9 & $\ldots$ & $\ldots$ & $\ldots$ & $\ldots$ & $\ldots$ & $\ldots$ \\
\hline MGRO J1958+2848 & PSR & 21 & 24 & 0 & 65.88 & -0.34 & $0.04,0.01$ & $<0.56$ \\
\hline $\mathrm{a}$ & & 8 & $\cdots$ & $\cdots$ & $\cdots$ & $\cdots$ & $\ldots$ & $\cdots$ \\
\hline VER J2016+372 & $\mathrm{O}$ & 31 & 33 & 1 & 74.86 & 1.22 & $0.05,0.02$ & $<1.16$ \\
\hline MGRO J2019+37 & PSR & 31 & 100 & 1 & 75.23 & 0.13 & $0.02,0.01$ & $<0.07$ \\
\hline a & & 5 & $\cdots$ & $\cdots$ & $\cdots$ & $\cdots$ & $\ldots$ & $\cdots$ \\
\hline MGRO J2031+41B & PSR & 58 & 66 & 5 & 80.20 & 1.03 & $0.05,0.01$ & $<2.47$ \\
\hline a & & 12 & $\cdots$ & $\cdots$ & $\ldots$ & $\ldots$ & $\ldots$ & $\ldots$ \\
\hline MGRO J2228+61 & PSR & 94 & 113 & 0 & 106.65 & 2.94 & $0.02,0.01$ & $<0.10$ \\
\hline $\mathrm{a}$ & & 15 & $\cdots$ & $\cdots$ & $\ldots$ & $\cdots$ & $\ldots$ & $\ldots$ \\
\hline
\end{tabular}

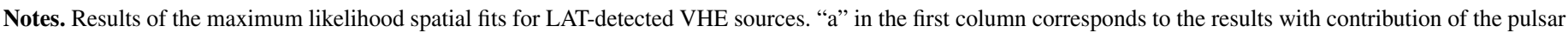

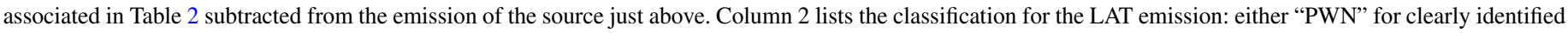

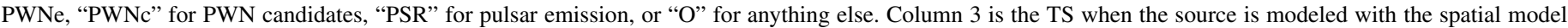

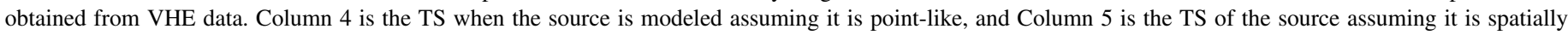

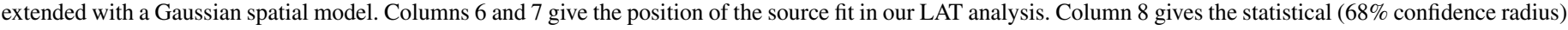

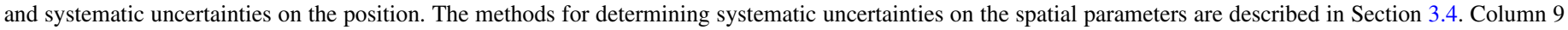
gives the extension fit in the LAT energy range if $\mathrm{TS}_{\mathrm{ext}}>16$ or a $99 \%$ c.l. upper limit on the extension otherwise.

The imperfect knowledge of the true $\gamma$-ray morphology introduces a last source of error. We derived an estimate of the uncertainty on the spectral parameters due to the uncertainty on the $\gamma$-ray morphology by computing the difference between the values obtained assuming the published VHE spatial model and those obtained assuming the best-fit $\mathrm{GeV}$ extension obtained in this analysis.
As discussed in Section 3.2, for the sources near LAT-detected pulsars, a fourth source of systematic uncertainties can affect the LAT flux estimate : the pulsar model. For these sources, we studied any potential contamination of the putative LAT $\gamma$-ray sources by pulsars by performing a second fit of the regions including the pulsars in our background models. The results are also included in Tables 4-6 and are flagged with an "a." 
Table 5

Spectral Results for Detected Sources

\begin{tabular}{|c|c|c|c|c|c|c|c|c|c|}
\hline Name & TS & $\begin{array}{c}F_{10 \mathrm{GeV}}^{316 \mathrm{GeV}} \\
\left(10^{-10} \mathrm{~cm}^{-2} \mathrm{~s}^{-1}\right)\end{array}$ & $\Gamma$ & $\mathrm{TS}_{10 \mathrm{GeV}}^{31 \mathrm{GeV}}$ & $\begin{array}{c}F_{10 \mathrm{GeV}}^{31 \mathrm{GeV}} \\
\left(10^{-10} \mathrm{~cm}^{-2} \mathrm{~s}^{-1}\right)\end{array}$ & $\mathrm{TS}_{31 \mathrm{GeV}}^{100 \mathrm{GeV}}$ & $\begin{array}{c}F_{31 \mathrm{GeV}}^{100 \mathrm{GeV}} \\
\left(10^{-10} \mathrm{~cm}^{-2} \mathrm{~s}^{-1}\right)\end{array}$ & $\mathrm{TS}_{100 \mathrm{GeV}}^{316 \mathrm{GeV}}$ & $\begin{array}{c}F_{100 \mathrm{GeV}}^{316 \mathrm{GeV}} \\
\left(10^{-10} \mathrm{~cm}^{-2} \mathrm{~s}^{-1}\right)\end{array}$ \\
\hline VER J0006 + 727 & 655 & $11.3 \pm 0.9 \pm 1.2$ & $3.96 \pm 0.25 \pm 0.36$ & 647 & $11.2 \pm 0.9 \pm 2.0$ & 11 & $0.4 \pm 0.2 \pm 0.2$ & 0 & $<0.3$ \\
\hline a & 2 & $<1.2$ & $\ldots$ & 3 & $<1.2$ & 0 & $<0.4$ & 0 & $<0.3$ \\
\hline MGRO J0632 + 17 & 699 & $34.9 \pm 2.1 \pm 10.2$ & $4.53 \pm 0.25 \pm 0.51$ & 695 & $36.8 \pm 2.0 \pm 10.1$ & 6 & $<1.6$ & 1 & $<0.7$ \\
\hline a & 5 & $<5.1$ & $\ldots$ & 9 & $<5.5$ & 1 & $<1.1$ & 0 & $<0.6$ \\
\hline HESS J1018-589 & 29 & $1.7 \pm 0.5 \pm 0.7$ & $2.41 \pm 0.49 \pm 0.49$ & 25 & $1.4 \pm 0.5 \pm 0.6$ & 0 & $<0.6$ & 6 & $<0.6$ \\
\hline a & 25 & $1.5 \pm 0.5 \pm 0.7$ & $2.31 \pm 0.50 \pm 0.49$ & 20 & $1.3 \pm 0.4 \pm 0.6$ & 0 & $<0.6$ & 6 & $<0.6$ \\
\hline HESS J1023-575 & 52 & $4.6 \pm 0.9 \pm 1.2$ & $1.99 \pm 0.24 \pm 0.32$ & 40 & $3.8 \pm 0.8 \pm 1.8$ & 2 & $<0.9$ & 9 & $<1.2$ \\
\hline a & 52 & $4.6 \pm 0.9 \pm 1.2$ & $1.99 \pm 0.24 \pm 0.32$ & 40 & $3.8 \pm 0.8 \pm 1.8$ & 2 & $<0.9$ & 9 & $<1.2$ \\
\hline HESS J1119-614 & 27 & $2.1 \pm 0.6 \pm 0.8$ & $2.15 \pm 0.37 \pm 0.36$ & 17 & $1.5 \pm 0.5 \pm 0.5$ & 1 & $<0.7$ & 11 & $0.2 \pm 0.1 \pm 0.1$ \\
\hline a & 16 & $2.0 \pm 0.6 \pm 0.8$ & $1.83 \pm 0.41 \pm 0.36$ & 5 & $<2.1$ & 1 & $<0.8$ & 11 & $0.2 \pm 0.1 \pm 0.1$ \\
\hline HESS J1303-631 & 37 & $3.6 \pm 0.9 \pm 2.1$ & $1.53 \pm 0.23 \pm 0.37$ & 10 & $1.6 \pm 0.7 \pm 1.5$ & 25 & $1.6 \pm 0.5 \pm 0.7$ & 3 & $<0.7$ \\
\hline HESS J1356-645 & 24 & $1.1 \pm 0.4 \pm 0.5$ & $0.95 \pm 0.40 \pm 0.40$ & 0 & $<0.9$ & 14 & $0.6 \pm 0.3 \pm 0.3$ & 10 & $0.3 \pm 0.2 \pm 0.2$ \\
\hline a & 24 & $1.1 \pm 0.4 \pm 0.5$ & $0.94 \pm 0.40 \pm 0.40$ & 0 & $<0.9$ & 14 & $0.6 \pm 0.3 \pm 0.3$ & 10 & $0.3 \pm 0.2 \pm 0.2$ \\
\hline HESS J1418-609 & 31 & $4.0 \pm 1.0 \pm 1.3$ & $3.52 \pm 0.81 \pm 0.61$ & 29 & $3.6 \pm 0.9 \pm 1.2$ & 2 & $<1.0$ & 0 & $<0.6$ \\
\hline a & 15 & $<4.3$ & $\ldots$ & 13 & $2.6 \pm 0.9 \pm 1.2$ & 2 & $<1.0$ & 0 & $<0.6$ \\
\hline HESS J1420-607 & 42 & $3.7 \pm 0.9 \pm 1.1$ & $1.89 \pm 0.28 \pm 0.31$ & 19 & $2.4 \pm 0.7 \pm 0.7$ & 13 & $0.8 \pm 0.3 \pm 0.3$ & 12 & $0.5 \pm 0.2 \pm 0.2$ \\
\hline a & 36 & $3.4 \pm 0.9 \pm 1.1$ & $1.81 \pm 0.29 \pm 0.31$ & 15 & $2.2 \pm 0.7 \pm 0.7$ & 13 & $0.8 \pm 0.3 \pm 0.3$ & 12 & $0.5 \pm 0.2 \pm 0.2$ \\
\hline HESS J1507-622 & 21 & $1.5 \pm 0.5 \pm 0.5$ & $2.33 \pm 0.48 \pm 0.48$ & 18 & $1.2 \pm 0.4 \pm 0.4$ & 3 & $<0.7$ & 0 & $<0.4$ \\
\hline HESS J1514-591 & 156 & $6.2 \pm 0.9 \pm 1.3$ & $1.72 \pm 0.16 \pm 0.17$ & 69 & $3.9 \pm 0.7 \pm 1.1$ & 54 & $1.7 \pm 0.4 \pm 0.4$ & 36 & $0.7 \pm 0.3 \pm 0.4$ \\
\hline HESS J1614-518 & 110 & $9.9 \pm 1.4 \pm 3.1$ & $1.75 \pm 0.15 \pm 0.18$ & 47 & $6.1 \pm 1.1 \pm 2.7$ & 37 & $2.6 \pm 0.6 \pm 0.8$ & 31 & $1.1 \pm 0.4 \pm 0.3$ \\
\hline HESS J1616-508 & 75 & $9.3 \pm 1.4 \pm 2.3$ & $2.18 \pm 0.19 \pm 0.20$ & 46 & $6.5 \pm 1.2 \pm 2.1$ & 29 & $2.3 \pm 0.6 \pm 0.6$ & 3 & $<1.0$ \\
\hline HESS J1632-478 & 137 & $11.8 \pm 1.5 \pm 5.3$ & $1.82 \pm 0.14 \pm 0.19$ & 69 & $7.8 \pm 1.2 \pm 4.2$ & 37 & $2.6 \pm 0.6 \pm 0.9$ & 39 & $1.5 \pm 0.4 \pm 0.5$ \\
\hline HESS J1634-472 & 33 & $5.6 \pm 1.3 \pm 2.5$ & $1.96 \pm 0.25 \pm 0.29$ & 20 & $3.6 \pm 1.0 \pm 2.1$ & 12 & $1.3 \pm 0.5 \pm 0.5$ & 2 & $<0.8$ \\
\hline HESS J1640-465 & 47 & $5.0 \pm 1.0 \pm 1.7$ & $1.95 \pm 0.23 \pm 0.20$ & 24 & $3.4 \pm 0.9 \pm 1.3$ & 28 & $1.7 \pm 0.5 \pm 0.5$ & 0 & $<0.5$ \\
\hline HESS J1708-443 & 722 & $24.5 \pm 1.7 \pm 3.5$ & $3.80 \pm 0.24 \pm 0.33$ & 714 & $23.9 \pm 1.6 \pm 3.0$ & 14 & $1.1 \pm 0.4 \pm 0.5$ & 6 & $<1.0$ \\
\hline a & 33 & $5.5 \pm 1.3 \pm 3.5$ & $2.13 \pm 0.31 \pm 0.33$ & 17 & $4.0 \pm 1.2 \pm 3.0$ & 11 & $1.0 \pm 0.4 \pm 0.5$ & 6 & $<1.0$ \\
\hline HESS J1804-216 & 138 & $14.2 \pm 1.6 \pm 3.1$ & $2.10 \pm 0.16 \pm 0.24$ & 91 & $10.1 \pm 1.4 \pm 2.3$ & 38 & $2.9 \pm 0.7 \pm 0.6$ & 21 & $1.2 \pm 0.4 \pm 0.4$ \\
\hline a & 124 & $13.4 \pm 1.6 \pm 3.1$ & $2.04 \pm 0.16 \pm 0.24$ & 77 & $9.3 \pm 1.4 \pm 2.3$ & 36 & $2.8 \pm 0.7 \pm 0.6$ & 21 & $1.2 \pm 0.4 \pm 0.4$ \\
\hline HESS J1825-137 & 56 & $5.6 \pm 1.2 \pm 9.0$ & $1.32 \pm 0.20 \pm 0.39$ & 10 & $1.7 \pm 0.9 \pm 1.5$ & 30 & $2.9 \pm 0.7 \pm 1.6$ & 17 & $0.8 \pm 0.3 \pm 0.8$ \\
\hline HESS J1834-087 & 27 & $5.5 \pm 1.2 \pm 2.5$ & $2.24 \pm 0.34 \pm 0.42$ & 19 & $4.2 \pm 1.1 \pm 1.9$ & 7 & $<1.9$ & 3 & $<1.0$ \\
\hline HESS J1837-069 & 73 & $7.5 \pm 1.3 \pm 4.2$ & $1.47 \pm 0.18 \pm 0.30$ & 28 & $4.3 \pm 1.1 \pm 3.5$ & 21 & $1.9 \pm 0.6 \pm 0.9$ & 27 & $1.4 \pm 0.5 \pm 0.5$ \\
\hline HESS J1841-055 & 64 & $10.9 \pm 0.8 \pm 4.1$ & $1.60 \pm 0.27 \pm 0.33$ & 20 & $7.4 \pm 1.6 \pm 2.9$ & 13 & $2.1 \pm 0.7 \pm 1.0$ & 31 & $1.8 \pm 0.5 \pm 0.7$ \\
\hline HESS J1848-018 & 19 & $7.4 \pm 1.9 \pm 2.7$ & $2.46 \pm 0.50 \pm 0.51$ & 16 & $5.8 \pm 1.6 \pm 2.9$ & 4 & $<2.6$ & 0 & $<1.0$ \\
\hline HESS J1857 + 026 & 53 & $4.2 \pm 0.3 \pm 1.3$ & $1.01 \pm 0.24 \pm 0.25$ & 6 & $<2.9$ & 19 & $1.2 \pm 0.4 \pm 0.4$ & 31 & $1.5 \pm 0.4 \pm 0.4$ \\
\hline MGRO J1908 + 06 & 16 & $4.6 \pm 1.3 \pm 1.5$ & $3.50 \pm 1.11 \pm 1.60$ & 11 & $3.4 \pm 1.2 \pm 1.2$ & 2 & $<1.6$ & 7 & $<1.5$ \\
\hline a & 9 & $<5.6$ & $\ldots$ & 3 & $<3.9$ & 2 & $<1.6$ & 7 & $<1.5$ \\
\hline MGRO J1958 + 2848 & 21 & $1.3 \pm 0.4 \pm 0.7$ & $4.36 \pm 1.09 \pm 1.21$ & 19 & $1.3 \pm 0.5 \pm 0.5$ & 0 & $<0.3$ & 0 & $<0.3$ \\
\hline a & 8 & $<1.8$ & $\ldots$ & 15 & $1.2 \pm 0.5 \pm 0.5$ & 0 & $<0.3$ & 0 & $<0.3$ \\
\hline VER J2016 + 372 & 31 & $1.8 \pm 0.5 \pm 0.8$ & $2.45 \pm 0.44 \pm 0.49$ & 26 & $1.6 \pm 0.5 \pm 0.5$ & 3 & $<0.6$ & 3 & $<0.5$ \\
\hline MGRO J2019 + 37 & 31 & $4.4 \pm 1.7 \pm 1.1$ & $6.37 \pm 3.04 \pm 1.21$ & 26 & $5.4 \pm 1.2 \pm 1.8$ & 0 & $<0.7$ & 1 & $<0.8$ \\
\hline a & 5 & $<4.7$ & $\ldots$ & 9 & $<5.3$ & 0 & $<0.8$ & 1 & $<0.8$ \\
\hline MGRO J2031 + 41B & 58 & $6.4 \pm 1.0 \pm 1.1$ & $3.07 \pm 0.33 \pm 0.37$ & 58 & $6.2 \pm 1.0 \pm 1.3$ & 3 & $<1.0$ & 0 & $<0.4$ \\
\hline a & 12 & $<4.4$ & $\cdots$ & 11 & $3.0 \pm 0.9 \pm 1.0$ & 1 & $<0.9$ & 0 & $<0.4$ \\
\hline MGRO J2228 + 61 & 94 & $2.8 \pm 0.5 \pm 0.5$ & $3.06 \pm 0.41 \pm 0.42$ & 87 & $2.5 \pm 0.5 \pm 0.5$ & 7 & $<0.8$ & 0 & $<0.2$ \\
\hline a & 15 & $<2.0$ & $\ldots$ & 9 & $<1.8$ & 7 & $<0.8$ & 0 & $<0.2$ \\
\hline
\end{tabular}

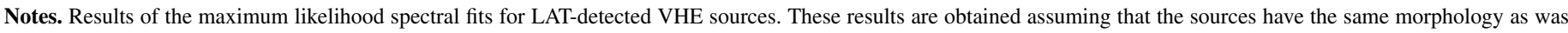

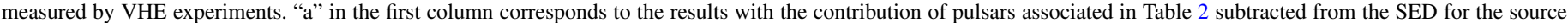

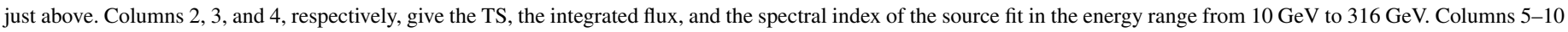

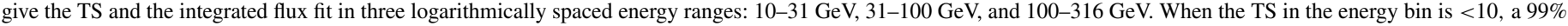
c.l. upper limit on the flux is given instead. The two uncertainties respectively correspond to the statistical and systematic uncertainties.

The effects of pulsar contamination are discussed for individual sources in Section 4.

We caution that some LAT-detected pulsars may have spectra at LAT energies that deviate from the simple exponential cutoff power-law model assumed in the 2FGL catalog, for energies above $10 \mathrm{GeV}$. Aliu et al. (2011) show this to be the case for the Crab pulsar. In such cases, our fit could still be contaminated by the pulsar, especially in the lowest energy bin between 10 and $31.6 \mathrm{GeV}$. For instance, in the case of Geminga for which this analysis detected no significant PWN-like emission, the comparison of the energy flux above $10 \mathrm{GeV}$ between the 2FGL and the 1FHL catalogs shows a factor $\sim 2$ smaller flux for $2 \mathrm{FGL}$.

Table 5 shows that HESS J1825-137 and MGRO J0632+17 have large systematic uncertainties. The uncertainty on HESS
J1825-137 mainly comes from the term corresponding to the source morphology. As can be seen in Tables 1 and 4, the LAT best-fit Gaussian has $\sigma=0.44$ while the VHE symmetric Gaussian has $\sigma=0.125$. This difference of morphology yields a difference in maximum likelihood flux of $+155 \%$.

The large systematic uncertainty on MGRO J0632+17 is not surprising since the VHE source has an extension of more than $1^{\circ}$, while the LAT morphology is best fit as a point source located at the pulsar's position.

\section{ANALYSIS RESULTS}

We detected 30 statistically significant LAT $\gamma$-ray sources among the $58 \mathrm{VHE}$ sources. The results of the spatial and 
Table 6

Spectral Results for Undetected Sources

\begin{tabular}{|c|c|c|c|c|c|c|c|c|}
\hline Name & $\mathrm{TS}$ & $\begin{array}{c}F_{10 \mathrm{GeV}}^{316 \mathrm{GeV}} \\
\left(10^{-10} \mathrm{~cm}^{-2} \mathrm{~s}^{-1}\right)\end{array}$ & $\mathrm{TS}_{10 \mathrm{GeV}}^{31 \mathrm{GeV}}$ & $\begin{array}{c}F_{10 \mathrm{GeV}}^{31 \mathrm{GeV}} \\
\left(10^{-10} \mathrm{~cm}^{-2} \mathrm{~s}^{-1}\right)\end{array}$ & $\mathrm{TS}_{31 \mathrm{GeV}}^{100 \mathrm{GeV}}$ & $\begin{array}{c}F_{31 \mathrm{GeV}}^{100 \mathrm{GeV}} \\
\left(10^{-10} \mathrm{~cm}^{-2} \mathrm{~s}^{-1}\right)\end{array}$ & $\mathrm{TS}_{100 \mathrm{GeV}}^{316 \mathrm{GeV}}$ & $\begin{array}{c}F_{100 \mathrm{GeV}}^{316 \mathrm{GeV}} \\
\left(10^{-10} \mathrm{~cm}^{-2} \mathrm{~s}^{-1}\right)\end{array}$ \\
\hline MGRO J0631 + 105 & 6 & $<1.4$ & 4 & $<1.2$ & 3 & $<0.6$ & 0 & $<0.4$ \\
\hline a & 2 & $<1.0$ & 0 & $<0.8$ & 3 & $<0.6$ & 0 & $<0.4$ \\
\hline HESS J1026-582 & 1 & $<1.6$ & 0 & $<1.6$ & 0 & $<0.4$ & 6 & $<0.7$ \\
\hline a & 1 & $<1.6$ & 0 & $<1.6$ & 0 & $<0.4$ & 6 & $<0.7$ \\
\hline HESS J1427-608 & 5 & $<1.9$ & 0 & $<1.2$ & 2 & $<0.8$ & 4 & $<0.6$ \\
\hline HESS J1458-608 & 13 & $<2.6$ & 13 & $1.7 \pm 0.5 \pm 0.7$ & 0 & $<0.5$ & 0 & $<0.3$ \\
\hline a & 13 & $<2.6$ & 13 & $1.7 \pm 0.5 \pm 0.7$ & 0 & $<0.5$ & 0 & $<0.3$ \\
\hline HESS J1503-582 & 10 & $<3.9$ & 1 & $<2.2$ & 4 & $<1.5$ & 9 & $<1.0$ \\
\hline HESS J1554-550 & 0 & $<0.5$ & 0 & $<0.6$ & 0 & $<0.3$ & 0 & $<0.3$ \\
\hline HESS J1626-490 & 1 & $<2.3$ & 1 & $<2.0$ & 1 & $<1.0$ & 0 & $<0.6$ \\
\hline HESS J1646-458A & 0 & $<2.8$ & 0 & $<1.7$ & 1 & $<1.9$ & 1 & $<1.0$ \\
\hline a & 0 & $<2.7$ & 0 & $<1.7$ & 1 & $<1.9$ & 1 & $<1.0$ \\
\hline HESS J1646-458B & 6 & $<4.7$ & 0 & $<2.6$ & 4 & $<1.9$ & 5 & $<1.2$ \\
\hline a & 4 & $<4.3$ & 0 & $<2.2$ & 4 & $<1.9$ & 5 & $<1.3$ \\
\hline HESS J1702-420 & 6 & $<4.7$ & 0 & $<2.6$ & 8 & $<2.4$ & 1 & $<0.8$ \\
\hline a & 6 & $<4.5$ & 0 & $<2.5$ & 8 & $<2.4$ & 1 & $<0.8$ \\
\hline HESS J1718-385 & 3 & $<2.1$ & 0 & $<1.5$ & 0 & $<0.8$ & 6 & $<0.9$ \\
\hline a & 3 & $<2.1$ & 0 & $<1.5$ & 0 & $<0.8$ & 6 & $<0.9$ \\
\hline HESS J1729-345 & 0 & $<1.4$ & 0 & $<1.4$ & 0 & $<0.8$ & 0 & $<0.5$ \\
\hline HESS J1809-193 & 15 & $<8.5$ & 11 & $4.7 \pm 1.5 \pm 2.1$ & 3 & $<2.3$ & 1 & $<1.0$ \\
\hline HESS J1813-178 & 3 & $<2.5$ & 0 & $<1.4$ & 5 & $<1.6$ & 1 & $<0.7$ \\
\hline HESS J1818-154 & 0 & $<1.6$ & 0 & $<1.6$ & 0 & $<0.8$ & 0 & $<0.4$ \\
\hline HESS J1831-098 & 0 & $<1.9$ & 0 & $<1.5$ & 0 & $<1.0$ & 0 & $<0.6$ \\
\hline HESS J1833-105 & 4 & $<2.1$ & 3 & $<1.9$ & 1 & $<0.7$ & 0 & $<0.7$ \\
\hline a & 4 & $<2.1$ & 3 & $<1.9$ & 1 & $<0.7$ & 0 & $<0.7$ \\
\hline HESS J1843-033 & 0 & $<0.9$ & 0 & $<1.0$ & 0 & $<0.5$ & 0 & $<0.5$ \\
\hline MGRO J1844-035 & 0 & $<1.4$ & 0 & $<1.2$ & 0 & $<0.9$ & 0 & $<0.5$ \\
\hline HESS J1846-029 & 2 & $<2.0$ & 5 & $<2.4$ & 0 & $<0.5$ & 0 & $<0.4$ \\
\hline HESS J1849-000 & 0 & $<1.3$ & 1 & $<1.5$ & 0 & $<0.5$ & 0 & $<0.5$ \\
\hline HESS J1858 + 020 & 0 & $<1.1$ & 0 & $<1.2$ & 0 & $<0.5$ & 0 & $<0.4$ \\
\hline MGRO J1900 + 039 & 0 & $<1.2$ & 0 & $<1.3$ & 0 & $<0.6$ & 0 & $<0.4$ \\
\hline HESS J1912 + 101 & 10 & $<4.6$ & 2 & $<2.7$ & 8 & $<2.0$ & 4 & $<1.1$ \\
\hline VER J1930 + 188 & 0 & $<1.0$ & 1 & $<1.1$ & 0 & $<0.4$ & 0 & $<0.3$ \\
\hline VER J1959 + 208 & 0 & $<0.3$ & 0 & $<0.3$ & 0 & $<0.3$ & 0 & $<0.4$ \\
\hline MGRO J2031 + 41A & 14 & $<29.6$ & 9 & $<22.6$ & 3 & $<8.4$ & 4 & $<2.3$ \\
\hline W49A & 3 & $<2.4$ & 0 & $<1.6$ & 3 & $<1.0$ & 3 & $<0.8$ \\
\hline
\end{tabular}

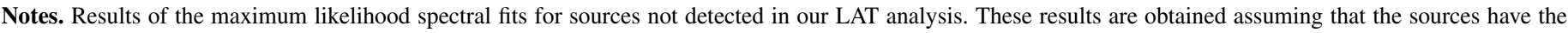

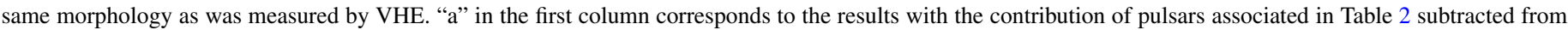

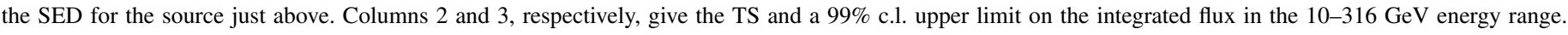

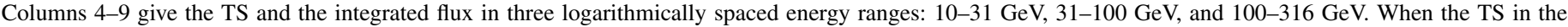
energy bin is $<10$, a $99 \%$ c.l. upper limit on the flux is given. The two uncertainties respectively correspond to the statistical and systematic uncertainties.

spectral analyses are shown in Tables 4 and 5. In addition to describing the LAT data analysis, we attempt to classify the origin of the GeV emission using the spatial and spectral information from LAT data as well as multi-wavelength information. We labeled each source as "PWN" when there is a clear PWN identification, "PWNc" when the source is a PWN candidate, "PSR" when the emission is likely coming from the pulsar only, and " $O$ " for other emission.

Table 7 summarizes the PSR/PWN systems' characteristics such as the age or the distance. The characteristic age of the pulsar is given by $\tau_{C}=P / 2 \dot{P}$, where $P$ is the pulsar's rotational period. This formula is obtained assuming that the pulsar is a dipole and that the initial period of the pulsar is negligible compared to its current value. The putative pulsars powering HESS J1554-550, HESS J1632-478, HESS J1640-465, HESS J1818-154, and HESS J1848-018 are not yet detected. However, Table 7 gives the system age or distance estimated using another method than the pulsar dispersion measure, e.g., distance measure derived by $\mathrm{H}$ I absorption or using a statistical relation between the hydrogen column density $\left(N_{\mathrm{H}}\right)$ and $E(B-V)$ (Ryter et al. 1975) and a relation between $E(B-V)$ and the distance (Lucke 1978).

\subsection{Spatial Results}

Assigning an association between an LAT source and a VHE source depends on two considerations: the spatial and spectral consistency with the counterpart. Spatial consistency has two aspects: centroid coincidence and extension compatibility. For assessing the degree of spatial coincidence, we considered an LAT emission to be spatially coincident with the VHE source if the distance between the LAT and VHE experiments' bestfit positions was inside a circle whose radius is the quadratic sum of (1) the $68 \%$ c.l. uncertainty on the LAT best-fit position, (2) the $68 \%$ c.l. position uncertainty of the VHE source, and (3) the $99 \%$ containment radius of the source based on the VHEfitted angular extent. These data are summarized in Table 8. 
Table 7

Pulsar and PWN Characteristics

\begin{tabular}{|c|c|c|c|c|c|c|}
\hline Name & PSR & $\begin{array}{c}\dot{E} \\
\left(\mathrm{erg} \mathrm{s}^{-1}\right)\end{array}$ & $\begin{array}{c}\tau_{C} \\
(\mathrm{kyr})\end{array}$ & $\begin{array}{l}\text { Distance } \\
(\mathrm{kpc})\end{array}$ & References & $\gamma$-Ray Pulsar \\
\hline VER J0006+727 & PSR J0007+7303 & $4.5 \mathrm{e}+35$ & 13.9 & $1.4 \pm 0.3$ & (1) & $\mathrm{Y}$ \\
\hline Crab & PSR J0534 + 2200 & $4.6 e+38$ & 1.2 & $2.0 \pm 0.5$ & (2) & $\mathrm{Y}$ \\
\hline MGRO J0631+105 & PSR J0631+1036 & $1.7 \mathrm{e}+35$ & 43.6 & $1.0 \pm 0.2$ & (3) & $\mathrm{Y}$ \\
\hline MGRO J0632+17 & PSR J0633+1746 & $3.2 \mathrm{e}+34$ & 342 & $0.2_{-0.1}^{+0.2}$ & (4) & $\mathrm{Y}$ \\
\hline Vela X & PSR J0835-4510 & $6.9 \mathrm{e}+36$ & 11.3 & $0.29 \pm 0.02$ & (5) & $\mathrm{Y}$ \\
\hline HESS J1018-589 & PSR J1016-5857 & $2.6 \mathrm{e}+36$ & 21 & $2.9_{-1.9}^{+0.6 \mathrm{a}}$ & $(6,7)$ & $\mathrm{Y}$ \\
\hline HESS J1023-575 & PSR J1023-5746 & $1.1 \mathrm{e}+37$ & 4.6 & $2.8^{\mathrm{b}}$ & (8) & $\mathrm{Y}$ \\
\hline HESS J1026-582 & PSR J1028-5819 & $8.4 e+35$ & 90 & $2.3 \pm 0.3$ & (9) & $\mathrm{Y}$ \\
\hline HESS J1119-614 & PSR J1119-6127 & $2.3 e+36$ & 1.6 & $8.4 \pm 0.4$ & (10) & $\mathrm{Y}$ \\
\hline HESS J1303-631 & PSR J1301-6305 & $1.7 \mathrm{e}+36$ & 11 & $6.7_{-1.2}^{+1.1}$ & $(11,12)$ & $\mathrm{N}$ \\
\hline HESS J1356-645 & PSR J1357-6429 & $3.1 \mathrm{e}+36$ & 7.3 & $2.5_{-0.4}^{+0.5}$ & (13) & $\mathrm{Y}$ \\
\hline HESS J1418-609 & PSR J1418-6058 & $4.9 \mathrm{e}+36$ & 1.0 & $1.6 \pm 0.7$ & (14) & $\mathrm{Y}$ \\
\hline HESS J1420-607 & PSR J1420-6048 & $1.0 \mathrm{e}+37$ & 13 & $5.6 \pm 0.9$ & $(11,15)$ & $\mathrm{Y}$ \\
\hline HESS J1458-608 & PSR J1459-6053 & $9.1 \mathrm{e}+35$ & 64.7 & $4^{\mathrm{c}}$ & (16) & $\mathrm{Y}$ \\
\hline HESS J1514-591 & PSR J1513-5908 & $1.7 \mathrm{e}+37$ & 1.56 & $4.2 \pm 0.6$ & (12) & $\mathrm{Y}$ \\
\hline HESS J1554-550 & $\ldots$ & $\ldots$ & $18^{\mathrm{d}}$ & $7.8 \pm 1.3^{\mathrm{e}}$ & $(17,18,19)$ & $\mathrm{N}$ \\
\hline HESS J1616-508 & PSR J1617-5055 & $1.6 e+37$ & 8.13 & $6.8 \pm 0.7^{\mathrm{f}}$ & (12) & $\mathrm{N}$ \\
\hline HESS J1632-478 & $\ldots$ & $3.0 \mathrm{e}+36^{\mathrm{g}}$ & 20 & $3.0^{c}$ & (20) & $\mathrm{N}$ \\
\hline HESS J1640-465 & $\ldots$ & $4.0 \mathrm{e}+36^{\mathrm{g}}$ & $\ldots$ & $\ldots$ & $(22,23)$ & $\mathrm{N}$ \\
\hline HESS J1646-458B & PSR J1648-4611 & $2.1 \mathrm{e}+35$ & 110 & $5.0 \pm 0.7$ & (21) & $\mathrm{Y}$ \\
\hline HESS J1702-420 & PSR J1702-4128 & $3.4 \mathrm{e}+35$ & 55 & $4.8 \pm 0.6$ & (24) & $\mathrm{Y}$ \\
\hline HESS J1708-443 & PSR J1709-4429 & $3.4 \mathrm{e}+36$ & 17.5 & $2.3 \pm 0.3$ & $(25)$ & $\mathrm{Y}$ \\
\hline HESS J1718-385 & PSR J1718-3825 & $1.3 e+36$ & 89.5 & $3.6 \pm 0.4$ & $(26,12)$ & $\mathrm{Y}$ \\
\hline HESS J1804-216 & PSR J1803-2137 & $2.2 \mathrm{e}+36$ & 16 & $3.8_{-0.5}^{+0.4}$ & (12) & $\mathrm{N}$ \\
\hline HESS J1809-193 & PSR J1809-1917 & $1.8 \mathrm{e}+36$ & 51.3 & $3.5 \pm 0.4^{\mathrm{f}}$ & (12) & $\mathrm{N}$ \\
\hline HESS J1813-178 & PSR J1813-1749 & $6.8 \mathrm{e}+37$ & 5.4 & $4.7^{\mathrm{h}}$ & $(12,27)$ & $\mathrm{N}$ \\
\hline HESS J1818-154 & PSR J1818-1541 & $2.3 e+33$ & $9^{\mathrm{i}}$ & $7.8_{-1.4}^{+1.6 \mathrm{f}}$ & (28) & $\mathrm{N}$ \\
\hline HESS J1825-137 & PSR J1826-1334 & $2.8 \mathrm{e}+36$ & 21 & $3.9 \pm 0.4$ & $(12,29)$ & $\mathrm{N}$ \\
\hline HESS J1831-098 & PSR J1831-0952 & $1.1 \mathrm{e}+36$ & 128 & $4.0 \pm 0.4^{\mathrm{f}}$ & $(12,30)$ & $\mathrm{N}$ \\
\hline HESS J1833-105 & PSR J1833-1034 & $3.4 \mathrm{e}+37$ & 4.85 & $4.7 \pm 0.4$ & (31) & $\mathrm{Y}$ \\
\hline HESS J1837-069 & PSR J1838-0655 & $5.5 \mathrm{e}+36$ & 2.23 & $6.6 \pm 0.9$ & (32) & $\mathrm{N}$ \\
\hline HESS J1841-055 & PSR J1838-0537 & $5.9 \mathrm{e}+36$ & 4.97 & $1.3^{\mathrm{b}}$ & (33) & $\mathrm{Y}$ \\
\hline HESS J1846-029 & PSR J1846-0258 & $8.1 \mathrm{e}+36$ & 0.73 & $5.1^{\mathrm{b}}$ & (12) & $\mathrm{N}$ \\
\hline HESS J1848-018 & $\ldots$ & $\ldots$ & $\ldots$ & $6^{c}$ & (34) & $\mathrm{N}$ \\
\hline HESS J1849-000 & PSR J1849-0001 & $9.8 \mathrm{e}+36$ & 42.9 & $7^{c}$ & (35) & $\mathrm{N}$ \\
\hline HESS J1857+026 & PSR J1856+0245 & $4.6 e+36$ & 20.6 & $9.0 \pm 1.2^{\mathrm{f}}$ & (12) & $\mathrm{N}$ \\
\hline MGRO J1908+06 & PSR J1907+0602 & $2.8 \mathrm{e}+36$ & 19.5 & $3.2 \pm 0.3$ & (36) & $\mathrm{Y}$ \\
\hline HESS J1912+101 & PSR J1913+1011 & $2.9 \mathrm{e}+36$ & 169 & $4.8_{-0.7}^{+0.5 \mathrm{f}}$ & (12) & $\mathrm{N}$ \\
\hline VER J1930+188 & PSR J1930+1852 & $1.2 \mathrm{e}+37$ & 2.89 & $9_{-2}^{+7} 1^{\mathrm{f}}$ & (37) & $\mathrm{N}$ \\
\hline MGRO J1958+2848 & PSR J1958+2846 & $3.4 \mathrm{e}+35$ & 21.7 & & (12) & $\mathrm{Y}$ \\
\hline VER J1959+208 & PSR J1959+2048 & $1.6 \mathrm{e}+35$ & $\ldots$ & $2.5 \pm 0.1$ & $(38,39)$ & $\mathrm{Y}$ \\
\hline MGRO J2019+37 & PSR J2021+3651 & $3.4 \mathrm{e}+36$ & 17.2 & $10_{-4}^{+2}$ & $(40,41)$ & $\mathrm{Y}$ \\
\hline MGRO J2228+61 & PSR J2229+6114 & $2.2 \mathrm{e}+37$ & 10.5 & $0.8 \pm 0.2$ & $(42,43)$ & $\mathrm{Y}$ \\
\hline
\end{tabular}

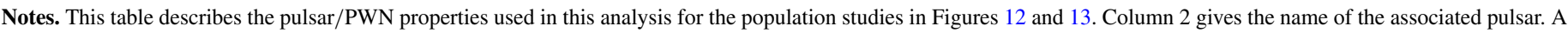

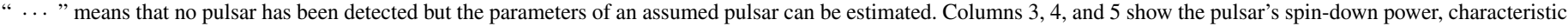

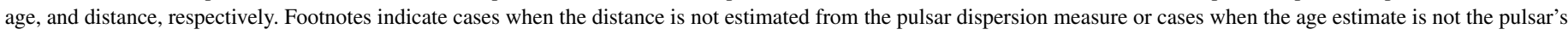
characteristic age. Column 7 says if the pulsar has been detected in $\gamma$-rays: "Y" means that it has and "N" means that it has not according to $2 \mathrm{PC}$.

a SNR distance.

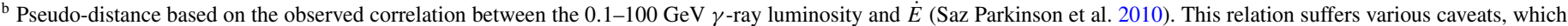
translates to large uncertainties in the derived distance value and is therefore replaced by a conservative upper limit in $2 \mathrm{PC}$.

${ }^{\mathrm{c}}$ No available uncertainty given in the reference.

d Sedov model; see Camilo et al. (2006).

${ }^{\mathrm{e}}$ Relation between the hydrogen column density $\left(N_{\mathrm{H}}\right)$ and $E(B-V)$ and between $E(B-V)$ and the distance.

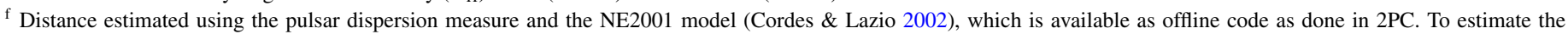
distance errors, we apply a $20 \%$ dispersion measure uncertainty as is used in the online DM-distance estimator tool.

g X-ray vs. spin-down luminosity correlation; see Hessels et al. (2004).

${ }^{\mathrm{h}}$ Distance derived from H I absorption.

${ }^{i}$ Sedov age; see de los Reyes et al. (2012).

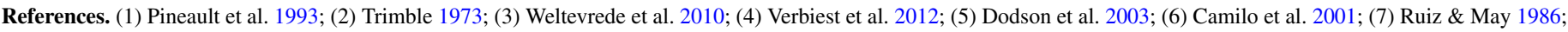

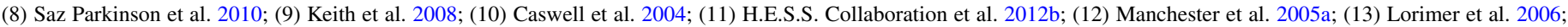

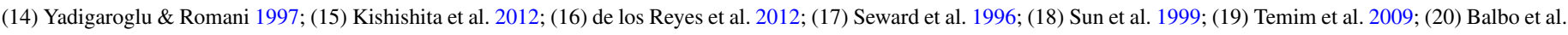

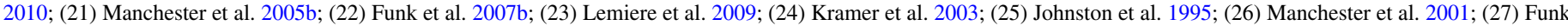

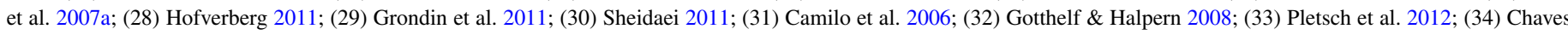

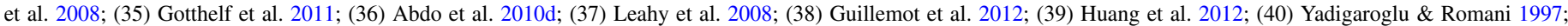
(41) Hessels et al. 2004; (42) Abdo et al. 2009b; (43) Kothes et al. 2001. 
Table 8

Comparison between the LAT and VHE Positions

\begin{tabular}{lcccc}
\hline \hline Name & $\begin{array}{c}\mathrm{Unc}_{\mathrm{GeV}} \\
(\mathrm{deg})\end{array}$ & $\begin{array}{c}\mathrm{Unc}_{\mathrm{TeV}} \\
(\mathrm{deg})\end{array}$ & $\begin{array}{c}r_{99 \%} \mathrm{TeV} \\
(\mathrm{deg})\end{array}$ & $\begin{array}{c}\text { Distance } \\
(\mathrm{deg})\end{array}$ \\
\hline HESS J1018-589 & 0.04 & 0.02 & 0.00 & 0.12 \\
HESS J1023-575 & 0.04 & 0.09 & 0.55 & 0.10 \\
HESS J1119-614 & 0.05 & $\ldots$ & 0.15 & 0.09 \\
HESS J1303-631 & 0.05 & 0.01 & 0.49 & 0.41 \\
HESS J1356-645 & 0.05 & 0.03 & 0.61 & 0.19 \\
HESS J1420-607 & 0.04 & 0.02 & 0.18 & 0.01 \\
HESS J1507-622 & 0.06 & 0.04 & 0.46 & 0.21 \\
HESS J1514-591 & 0.03 & 0.07 & 0.23 & 0.06 \\
HESS J1614-518 & 0.05 & 0.03 & 0.58 & 0.19 \\
HESS J1616-508 & 0.04 & 0.01 & 0.42 & 0.16 \\
HESS J1632-478 & 0.04 & 0.04 & 0.41 & 0.15 \\
HESS J1634-472 & 0.03 & 0.05 & 0.33 & 0.18 \\
HESS J1640-465 & 0.05 & 0.01 & 0.12 & 0.07 \\
HESS J1804-216 & 0.04 & 0.02 & 0.65 & 0.06 \\
HESS J1825-137 & 0.06 & 0.03 & 0.38 & 0.28 \\
HESS J1834-087 & 0.05 & 0.02 & 0.27 & 0.06 \\
HESS J1837-069 & 0.06 & 0.02 & 0.26 & 0.12 \\
HESS J1841-055 & 0.06 & 0.05 & 1.00 & 0.22 \\
HESS J1848-018 & 0.04 & $\ldots$ & 0.97 & 0.11 \\
HESS J1857+026 & 0.04 & 0.05 & 0.29 & 0.14 \\
VER J2016+372 & 0.05 & $\ldots$ & $\ldots$ & 0.11 \\
\hline
\end{tabular}

Notes. Comparison of the localization and localization uncertainty for the sources observed at LAT and VHE energies and classified as "PWN," "PWNc," or "O." Columns 2, 3, 4, and 5, respectively, give the averaged uncertainty on the position obtained using Fermi-LAT data and assuming a point source if $\mathrm{TS}_{\mathrm{ext}}<16$ or a Gaussian if $\mathrm{TS}_{\text {ext }}>16$, the quadratic sum of the statistical (68\% confidence radius) and systematic uncertainties on the VHE position given in Table 1, the $99 \%$ containment radius of the VHE emission assuming the extension listed in Table 1 , and the distance between the positions of the source fit at LAT and VHE energies.

Only one source is not spatially coincident using this criterion: HESS J1018-589. We removed from this table the sources likely to be associated with pulsar emission. These sources will be discussed in Section 4.2.

Although VER J2016+372 is spatially extended as announced in Aliu (2011), neither the extension nor the uncertainties of the spatial parameters are yet available. There is little doubt that these parameters will show that the VERITAS and LAT emissions are spatially coincident. This case will be discussed in Section 4.4.

A finite measured extension of a $\gamma$-ray source is a direct way to discriminate between a pulsar and a PWN. Therefore, we searched for significant angular extensions of the LAT sources. Of the 21 sources not labeled as PSR in Section 4.2, 8 are significantly extended: HESS J1303-631, HESS J1614-518, HESS J1616-508, HESS J1632-478, HESS J1804-216, HESS J1825-137, HESS J1837-069, and HESS J1841-055. Six of them were already detected by Lande et al. (2012). Only HESS J1632-478 has an extension inconsistent with that work. For this source, Lande et al. (2012) measured an extension of $0.35 \pm 0.06$ assuming a disk, while we obtained $0.30 \pm 0.04$ for a Gaussian. Comparison between $68 \%$ containment radius of a disk and a Gaussian yields $\sigma_{\text {Disk }} \sim 1.84 \sigma_{\text {Gaussian }}$ (Lande et al. 2012), where $\sigma_{\text {Disk }}$ and $\sigma_{\text {Gaussian }}$, respectively, represent the disk radius and the Gaussian standard deviation. Therefore, for HESS $\mathrm{J} 1632-478$, our analysis led to an extension bigger than that obtained in Lande et al. (2012). This difference certainly comes from the three additional 2FGL sources (2FGL J1631.7-4720c, 2FGL J1630.2-4752, and 2FGL J1632.4-4820c) included in

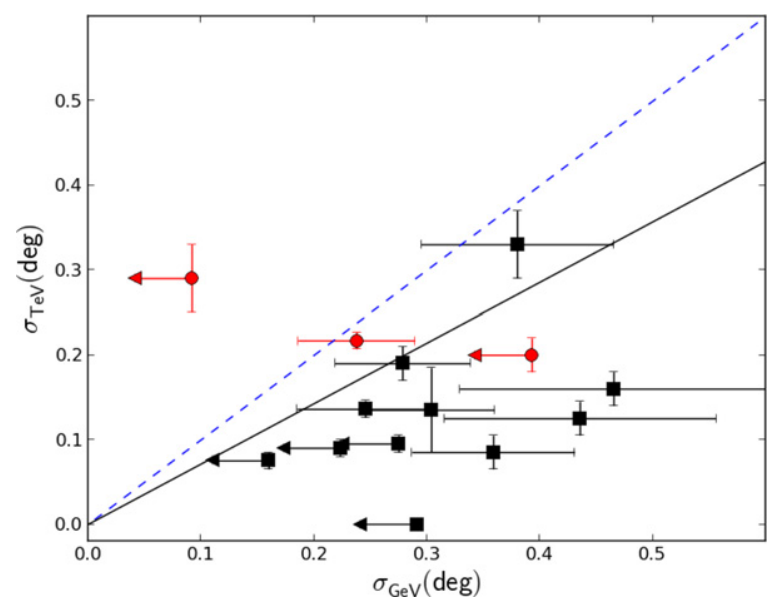

Figure 2. Comparison of the sizes of the sources observed at LAT and VHE energies. Contributions of pulsars listed in Table 2 were taken into account by modeling them as point sources with spectral parameters fixed at their values in the 2FGL catalog. For sources not significantly extended at LAT energies, a $99 \%$ c.l. upper limit on the extension is shown. The red circles represent the sources within 0.5 of a $\gamma$-ray pulsar, and the black squares represent the other sources. This plot shows a zoom on $\sigma_{\mathrm{TeV}}$ and $\sigma_{\mathrm{GeV}} \in\left[0^{\circ}, 0.6\right]$. The dashed line shows $\sigma_{\mathrm{TeV}}=\sigma_{\mathrm{GeV}}$. The solid line shows the $\sigma_{\mathrm{GeV}}=1.4 \times \sigma_{\mathrm{TeV}}$ fit result (see Section 4.1).

(A color version of this figure is available in the online journal.)

the model used by Lande et al. (2012). These sources are not included in the 1FHL catalog and are below the pre-defined TS $>25$ threshold for additional sources. Since they are unidentified, we chose not to add them.

Figure 2 compares the sizes measured by the LAT and VHE. The only source with $\sigma_{\mathrm{GeV}}<\sigma_{\mathrm{TeV}}$ is HESS J1708-443, whose LAT emission is likely coming from the pulsar. As we discussed above and in Section 4.5, we suspect that the extensions of HESS J1303-631 and HESS J1632-478 are overestimated. We assumed a linear relation between the VHE experiment and the LAT extension $\left(\sigma_{\mathrm{GeV}}=\alpha \times \sigma_{\mathrm{TeV}}\right)$ and fit the data points, obtaining $\alpha=1.4 \pm 0.2$ with a $\chi^{2} /$ dof $=15 / 7$. In the case in which we fixed $\alpha=1$ we obtained a $\chi^{2} /$ dof $=24 / 8$. This implies that, with the current statistics, the LAT extension is larger than the one measured by VHE experiments at the $2.8 \sigma$ level. Additional exposure and a more precise analysis of crowded regions (e.g., the regions of HESS J1303-631, HESS J1632-478, HESS J1837-069, or HESS J1841-055) are needed to draw any conclusion. Indeed, in these regions the $\gamma$-ray emission could be due to more than one source, as discussed below in the case of HESS J1841-055 or as discussed in Gotthelf \& Halpern (2008) for the case of HESS J1837-069.

\subsection{Pulsars Detected above $10 \mathrm{GeV}$}

In the LAT energy range, 22 sources are found to be pointlike, i.e., not significantly extended. Table 5 demonstrates that among these 22 point-like sources, 9 have a soft spectral index $(\Gamma>3)$ : HESS J1418-609, HESS J1708-443, MGRO J0632+17, MGRO J1908 + 06, MGRO J1958 + 2848, MGRO J2019+37, MGRO J2228+61, MGRO J2031+41B, and VER J0006+727. Tables 2 and 9 demonstrate that all of them are located close to an LAT-detected pulsar. Table 5 shows that their $\gamma$-ray emission significantly decreases when the contribution of the associated LAT-detected pulsar is included. Furthermore, the spectra obtained above $10 \mathrm{GeV}$ for these sources agree with the nearby pulsars' spectra given in the 2FGL catalog as shown in Figures 3-6. These figures only show SEDs for sources with 

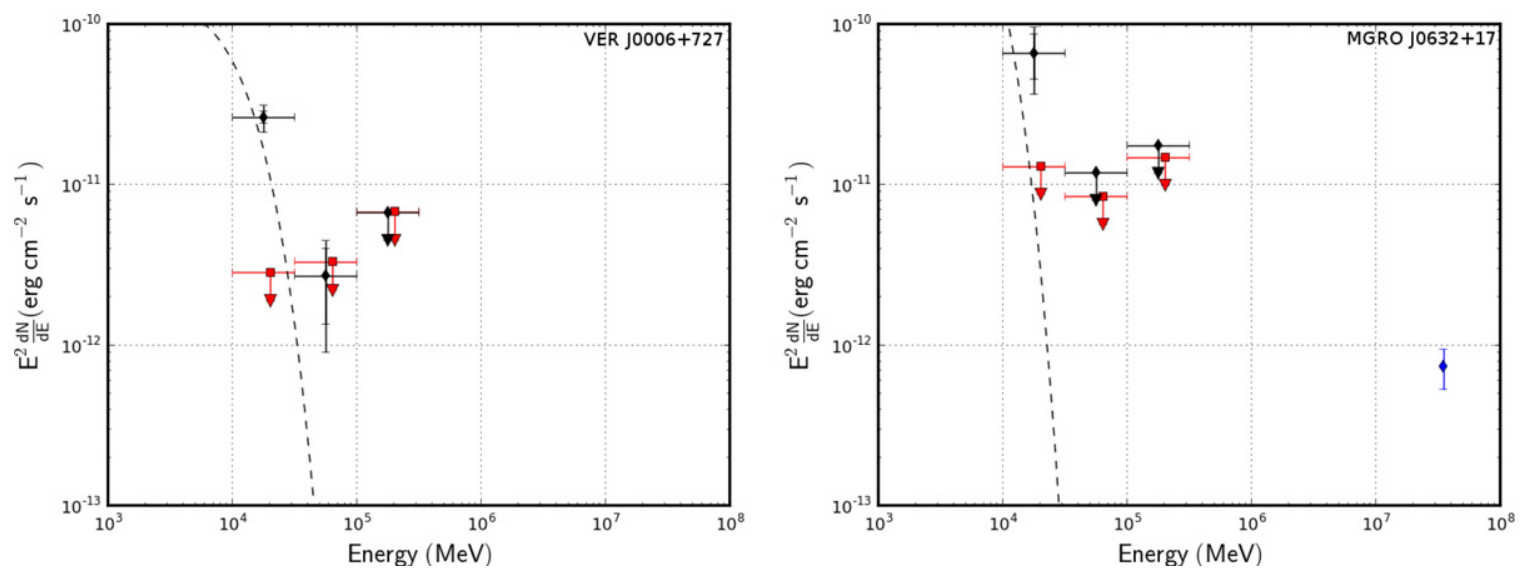

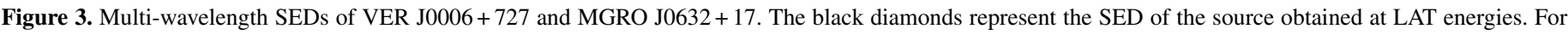

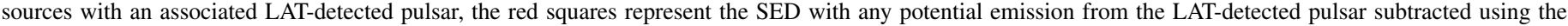

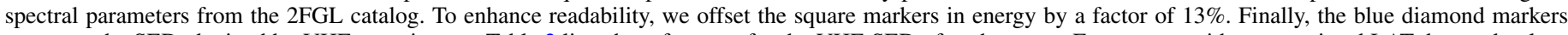

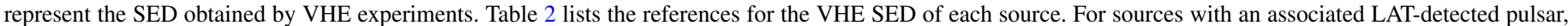
the best-fit spectrum of the pulsar from 2FGL is included as a dashed line.

(A color version of this figure is available in the online journal.)

Table 9

Comparison between the LAT Emission and Pulsar Positions

\begin{tabular}{lccc}
\hline \hline Name & $\begin{array}{c}\text { Unc }_{\mathrm{GeV}} \\
(\operatorname{arcmin})\end{array}$ & $\begin{array}{c}\text { Unc } \\
(\operatorname{arcmin})\end{array}$ & $\begin{array}{c}\text { Distance } \\
(\operatorname{arcmin})\end{array}$ \\
\hline VER J0006+727 & 1.2 & 0.4 & 1.2 \\
MGRO J0632+17 & 0.6 & 0.2 & 0.6 \\
HESS J1418-609 & 1.8 & 0.8 & 2.0 \\
HESS J1708-443 & 0.6 & 0.3 & 0.6 \\
MGRO J1908+06 & 2.4 & 0.8 & 2.5 \\
MGRO J1958+2848 & 2.4 & 1.1 & 1.2 \\
MGRO J2019+37 & 1.8 & 0.5 & 1.2 \\
MGRO J2031+41B & 3.6 & 0.8 & 1.2 \\
MGRO J2228+61 & 1.8 & 0.6 & 0.6 \\
\hline
\end{tabular}

Notes. For the nine sources classified as "PSR," a comparison of the localization of the source in the 10-316 GeV energy range with the localization in 2FGL from $100 \mathrm{MeV}$ to $100 \mathrm{GeV}$. Columns 2, 3, and 4, respectively, give the $68 \%$ uncertainty on the point-source position obtained in this work, the averaged $68 \%$ uncertainty on the 2FGL position, and the distance between the 2FGL position and the position obtained in this work. The average on the 2FGL position uncertainty is obtained as $\sqrt{a \times b}$, where $a$ and $b$ are the length of the semi-minor and semi-major axes of the $68 \%$ confidence ellipse defined in 2FGL.

no X-ray and radio information available. The spectra for the other sources will be discussed in Section 4.5.

To study the contamination of the LAT data for the VHE sources by these pulsars, we accounted for the pulsar contribution to photons in the region as explained in Section 3.2. Tables 4 and 5 and Figures 3-6 show the results of this new fit using the 2FGL models of the pulsars. For these nine sources, in Figures 3-6 the low-energy part of the emission $(<30 \mathrm{GeV})$ tends to disappear, suggesting that we primarily detected pulsar emission. For these reasons, we infer that the observed LAT emission coming from these sources is likely due to the pulsars themselves. We labeled these nine sources as "PSR" in Table 4.

\subsection{PWNe and PWN Candidates}

The hardness of the $\gamma$-ray spectrum and the presence of a pulsar energetic enough to power a potential PWN are important criteria for source classification as a PWN. In addition to these criteria, to label a source as a "PWN" or a "PWNc" for PWN candidate, we required that its LAT spectrum connects with the VHE spectrum, i.e., that the differential fluxes at the highest LAT energies and in the VHE low energy range be approximately equivalent, such that they can be interpreted as a continuous multi-wavelength spectrum. An exception has been made for HESS J1848-018, which is classified as "PWNc" as explained in Section 4.5.6. Based on multiwavelength analyses, we labeled three sources as "PWN": HESS J1356-645, HESS J1514-591, and HESS J1825-137. HESS J1356-645 is considered as "PWN" based upon the morphology of the source observed in the radio and X-ray domain (H.E.S.S. Collaboration et al. 2011c). HESS J1514-591 is considered as a "PWN" because of the correlation observed between the H.E.S.S. and X-ray emissions and the observation of pulsar jets in the X-ray domain (Aharonian et al. 2005b). And finally, HESS $\mathrm{J} 1825-137$ is considered to be a "PWN" since Aharonian et al. (2006b) have shown that it has an energy-dependent morphology in the H.E.S.S. energy range.

Detections of seven of the PWN candidates have been previously published. For HESS J1023-575 (Ackermann et al. 2011), HESS J1640-465 (Slane et al. 2010), HESS J1857+026 (Rousseau et al. 2012), HESS J1616-508, HESS J1632-478, HESS J1837-069 (Lande et al. 2012), and HESS J1848-018 (Tam et al. 2010), the LAT emission has been discussed in previous work and proposed to be of PWN origin.

We detected four new PWN candidates (HESS J1119-614, HESS J1303-631, HESS J1420-607, and HESS J1841-055) and one new PWN (HESS J1356-645). These sources are spatially consistent with pulsars able to power them (respectively, PSR J1119-6127, PSR J1301-6305, PSR J1420-6048, PSR J1838-0537, and PSR J1357-6429). Moreover, their LAT emissions are best modeled by hard spectra and are compatible with the VHE SEDs. The new LAT sources and PWN candidates are described in Section 4.5.

\section{4. “O”-type Sources}

The seven sources that cannot be associated with a PWN or a pulsar are labeled "O" for other. These sources cannot be associated because either no sufficiently energetic pulsar is 

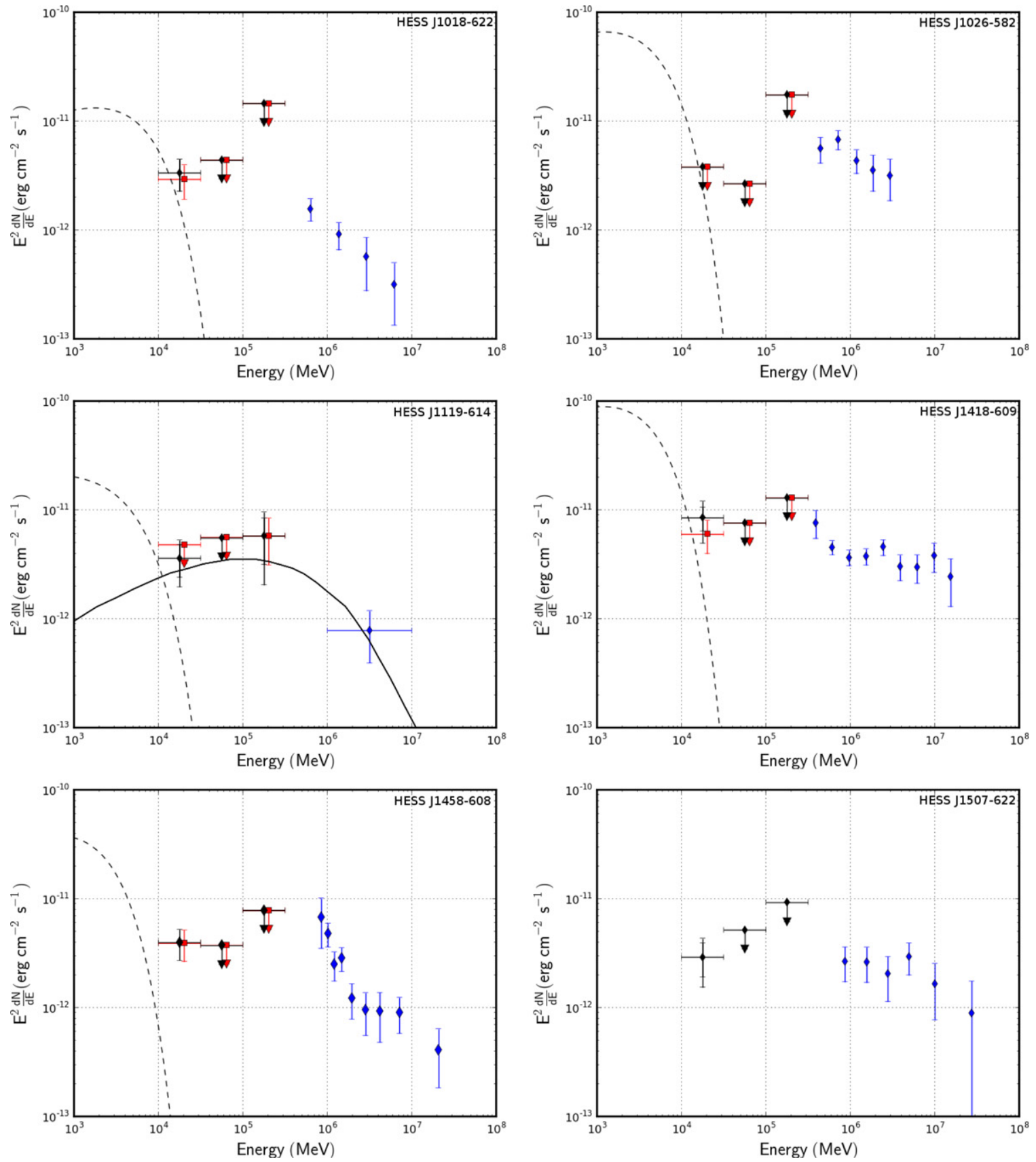

Figure 4. LAT and VHE SEDs of HESS J1018-589, HESS J1119-614, HESS J1418-609, HESS J1458-608, and HESS J1507-622 following conventions of Figure 3. The solid line corresponds to the model proposed by Mayer (2010) for HESS J1119-614. In the case of HESS J1119-614, the spectral point has been computed using the integral flux given in Kargaltsev et al. (2010; see Section 4.5 for details). The horizontal error bars show the energy range of the used integral flux. (A color version of this figure is available in the online journal.)

known at that location or the spectral connection from the LAT to the VHE measurements does not support a PWN interpretation.

Three sources have relatively hard spectra at LAT energies that connect spectrally to the associated VHE source: HESS J1614-518, HESS J1634-472, and HESS J1804-216. These sources are not classified as PWNe due to previous multiwavelength analyses. The former is spatially coincident with five detected pulsars, but none are luminous enough to power a PWN that would explain the $\gamma$-ray emission (Rowell et al. 2008). Sakai et al. (2011) found two X-ray counterparts to the H.E.S.S. source and proposed an SNR identification. As discussed in Lande et al. (2012), the nature of the source remains unclear. HESS
J1634-472 does not have any counterpart pulsars energetic enough to power it. Finally, Ajello et al. (2012) studied the link between the H.E.S.S. and the LAT emission coming from the region of HESS J1804-216 and concluded that the emission is more likely due to the energy-dependent scattering of particles accelerated in an SNR than to a PWN.

The remaining four sources are HESS J1018-589, HESS J1507-622, HESS J1834-087, and VER J2016+372. In the LAT energy range, they are point-like with relatively soft spectra $(3>\Gamma>2)$. Therefore, the association of the LAT emission with their VHE counterparts is uncertain. HESS J1018-589 is close to both the $\gamma$-ray binary 1FGL J1018.6-5856 (Coe 

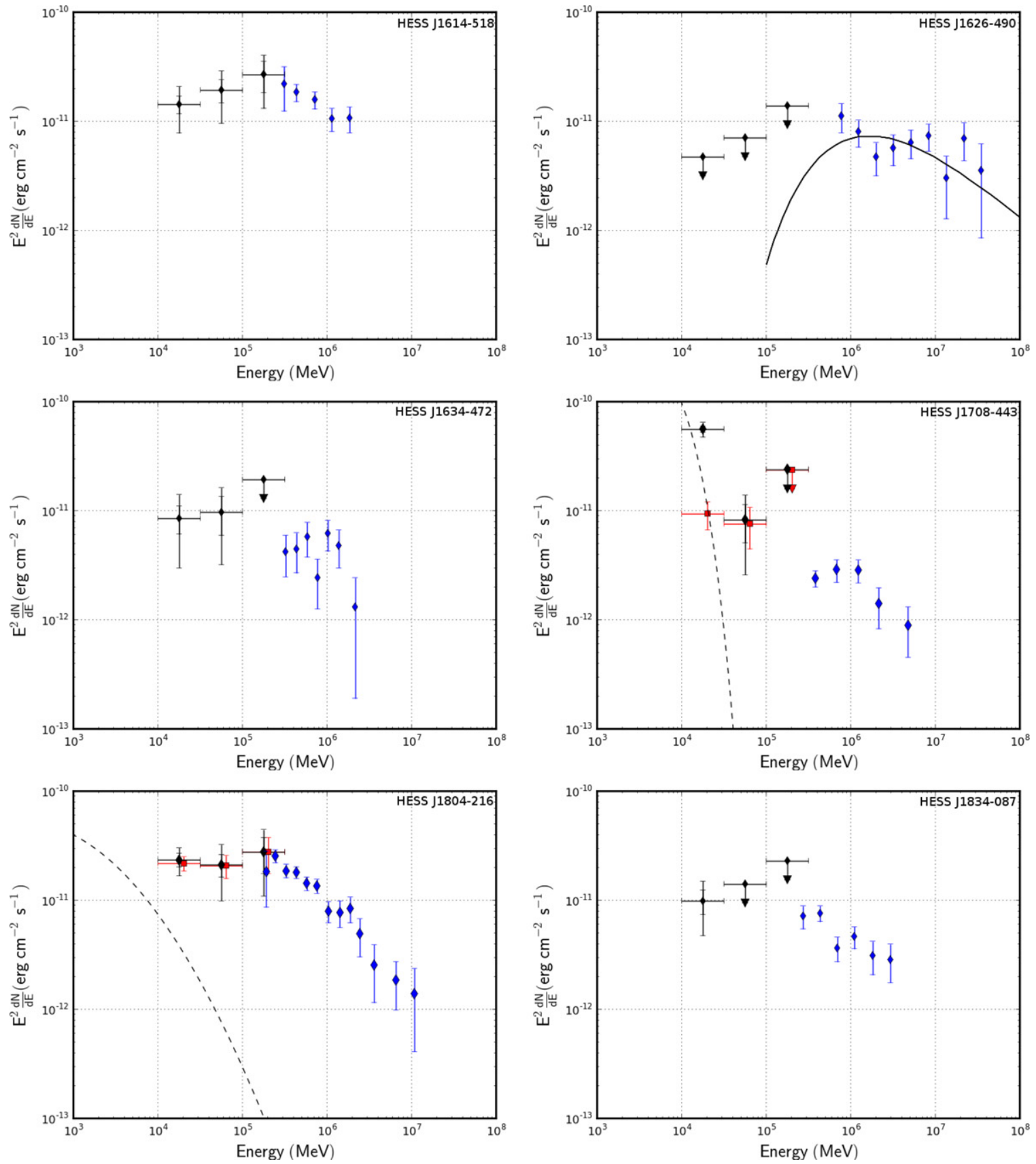

Figure 5. LAT and VHE SEDs of HESS J1614-518, HESS J1626-490, HESS J1634-472, HESS J1708-443, HESS J1804-216, and HESS J1834-087 following the conventions of Figure 3. The solid line corresponds to the model proposed by Eger (2011) for HESS J1626-490.

(A color version of this figure is available in the online journal.)

et al. 2012) and the nearby SNR G284.3-1.8. The LAT source appears to be spatially coincident with SNR G284.3-1.8. SNR G284.3-1.8 is not included in our list of candidates and will be analyzed in the SNR catalog. Domainko \& Ohm (2012) were not able to conclusively determine the origin of the $\gamma$-ray emission of HESS J1507-622 and confirm the result of H.E.S.S. Collaboration et al. (2011e): a leptonic scenario seems favored, while a hadronic one seems unlikely. HESS J1834-087 and VER J2016 + 372 lack a pulsar energetic enough to power their emission. But Albert et al. (2006b) have suggested that the interaction of SNR W41 with a nearby molecular cloud could explain HESS J1834-087. Another analysis of LAT and H.E.S.S. observations of HESS J1834-087 will be presented elsewhere.

\subsection{Descriptions of New LAT PWN Candidates}

In this section, we compare source spectra with previously published models (the references to those models are provided below for each case). We do not refit those models incorporating the LAT data. Therefore, disagreements between the published models and the LAT data do not necessarily imply that the models are ruled out as it may be possible to accommodate our results under these models with a different set of parameters.

$$
\text { 4.5.1. HESS J1119-614 }
$$

During the Parkes multibeam pulsar survey, Camilo et al. (2000) discovered PSR J1119-6127, a young $\left(\tau_{C}=1.6 \mathrm{kyr}\right.$ ) pulsar with a high spin-down power $\dot{E}=2.3 \times 10^{36} \mathrm{erg} \mathrm{s}^{-1}$ 

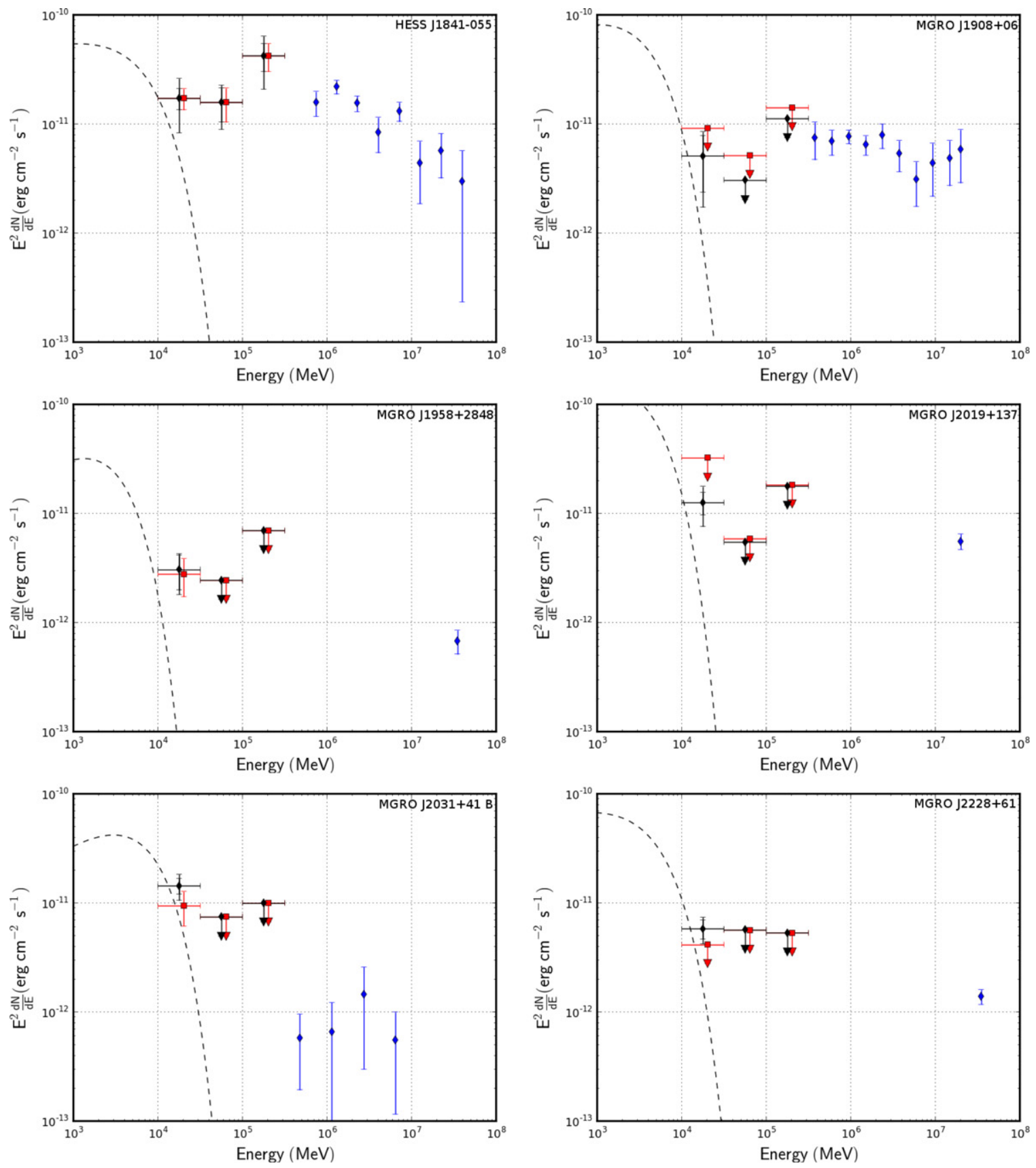

Figure 6. LAT and VHE SEDs of HESS J1841-055, MGRO J1908 + 06, MGRO J1958 + 2848, MGRO J2019 + 137, MGRO J2031 + 41B, and MGRO J2228 + 61 following the conventions of Figure 3.

(A color version of this figure is available in the online journal.)

within the composite SNR G292.2-0.5. This pulsar was also detected by the LAT (Parent et al. 2011). Using Chandra observations, Gonzalez \& Safi-Harb (2003) and Safi-Harb \& Kumar (2008) reported the presence of a faint and compact PWN close to this pulsar. More recently, an H.E.S.S. source coincident with PSR J1119-6127 and G292.2-0.5 was announced. ${ }^{67}$

Using the method described above, a signal is detected at the position of HESS J1119-614 with a TS of $27(4.9 \sigma$ with 2 dof). Nevertheless, as can be seen in Figure 4 and in Table 5, the spectrum is contaminated by a low-energy component associated with PSR J1119-6127. We assess the contamination from the pulsar by subtracting the emission from

\footnotetext{
67 http://cxc.harvard.edu/cdo/snr09/pres/DjannatiAtai_Arache_v2.pdf
}

2FGL J1118.8-6128 associated with PSR J1119-6127. This decreases the significance of the detection to TS $=16(3.6 \sigma$ with 2 dof), which is just above the threshold from Section 3.3. Subtracting the pulsar emission affects the spectrum of our source by making it harder and changing the lowest energy point to become an upper limit (see Figure 4).

Figure 4 shows the SED of HESS J1119-614. Because only an integral flux between 1 and $10 \mathrm{TeV}$ is available for this source (Kargaltsev et al. 2010), we computed a spectral point at $3.16 \mathrm{TeV}$ assuming a fiducial spectral index of 2.4 in the H.E.S.S. energy range. We represented this point in the figure. The leptonic model proposed by Mayer (2010) is a one-zone model in which accelerated electrons cool radiatively by IC scattering on CMB photons, stellar photons from the vicinity and 
photons radiated by dust, and by synchrotron losses. It implies an initial period of the pulsar $P_{0}=21.4 \mathrm{~ms}$, an initial magnetic field inside the PWN $B_{0} \sim 400 \mu \mathrm{G}$ (leading to a current magnetic field of $\mathrm{B} \sim 32 \mu \mathrm{G}$ ), a braking index of $n=2.91$ (Camilo et al. 2000), and a conversion efficiency of rotational energy into relativistic particles of $\eta=0.3$. The leptonic model matches the new LAT data points.

The energetics of PSR J1119-6127, the detection of a compact PWN in X-rays, and the leptonic model proposed by Mayer (2010) support the hypothesis in which the LAT-H.E.S.S. emission originates in the PWN inside G292.2-0.5. Furthermore, the parameters derived in Mayer (2010) and the jet-like morphology in the X-ray data are reminiscent of the case of MSH 15-52 (Abdo et al. 2010a; Tamura et al. 1996).

\subsubsection{HESS J1303-631}

HESS J1303-631 was serendipitously discovered in 2004 (Aharonian et al. 2005c) during an observation campaign of the binary system PSR B1259-63. It is the first H.E.S.S. source classified as a UNID due to the lack of detected counterparts in radio and X-rays with Chandra (Mukherjee \& Halpern 2005). H.E.S.S. Collaboration et al. (2012b) found only one plausible counterpart in the vicinity of HESS J1303-631: PSR J1301-6305 with a spin-down power of $\dot{E}=1.70 \times$ $10^{36} \mathrm{erg} \mathrm{s}^{-1}$. The authors also presented the detection of a very weak X-ray PWN using XMM-Newton observations. This, with the energy-dependent morphology observed by H.E.S.S., led to the conclusion that HESS J1303-631 is an old PWN offset from the pulsar powering it.

$\mathrm{Wu}$ et al. (2011) found no significant emission from HESS J1303-631 using 30 months of Fermi-LAT data between 1 and $20 \mathrm{GeV}$. With 15 months of additional data and a higher energy threshold, our analysis now provides a first detection of LAT emission coincident with the HESS source. Nevertheless, Figure 7 shows that the detected emission might be contaminated by $\gamma$-ray emission from the nearby SNR Kes 17. Since we cannot separate these two sources using our strategy with the current statistics, we decided to include the effect of source confusion in our estimate of systematic uncertainties for HESS J1303-631. Therefore, we ran the analysis again, adding a source at the position of Kes 17 . We quantify the systematic error as the differences in parameter values resulting from fitting with the two background models. The maximal variation is in the lowest energy bin of the SED.

For our best LAT morphology of a Gaussian of dispersion 0.45 (see Table 4), we obtained an index of $\Gamma=1.71 \pm 0.26 \pm$ 0.37 (we obtained $\Gamma=1.53 \pm 0.23 \pm 0.37$ assuming the H.E.S.S. best fit Gaussian; Table 5). This hard index is in the range of values obtained for LAT-detected PWNe and is inconsistent with the spectral index of $\sim 2.4$ derived by $\mathrm{Wu}$ et al. (2011) for Kes 17. This is evidence that the $\gamma$-ray emission above $10 \mathrm{GeV}$ is dominated by the PWN candidate. As can be seen in Figure 8, even though the connection between the LAT and the H.E.S.S. energy range is not perfect, LAT and H.E.S.S. spectra are not inconsistent, suggesting a potential physical relationship.

Figure 8 shows the SED of HESS J1303-631 together with the one-zone leptonic model proposed by H.E.S.S. Collaboration et al. (2012b). In this model, VHE $\gamma$-rays are created via IC scattering of electrons on the CMB photons. Infrared and optical target photons are neglected. The model reproduces the radio, X-ray, and H.E.S.S. data with an electron spectral index of $1.8 \pm 0.1$, a cutoff energy of $31_{-4}^{+5} \mathrm{TeV}$, and an average magnetic field of $1.4 \pm 0.2 \mu \mathrm{G}$. However, the flux predicted in the LAT
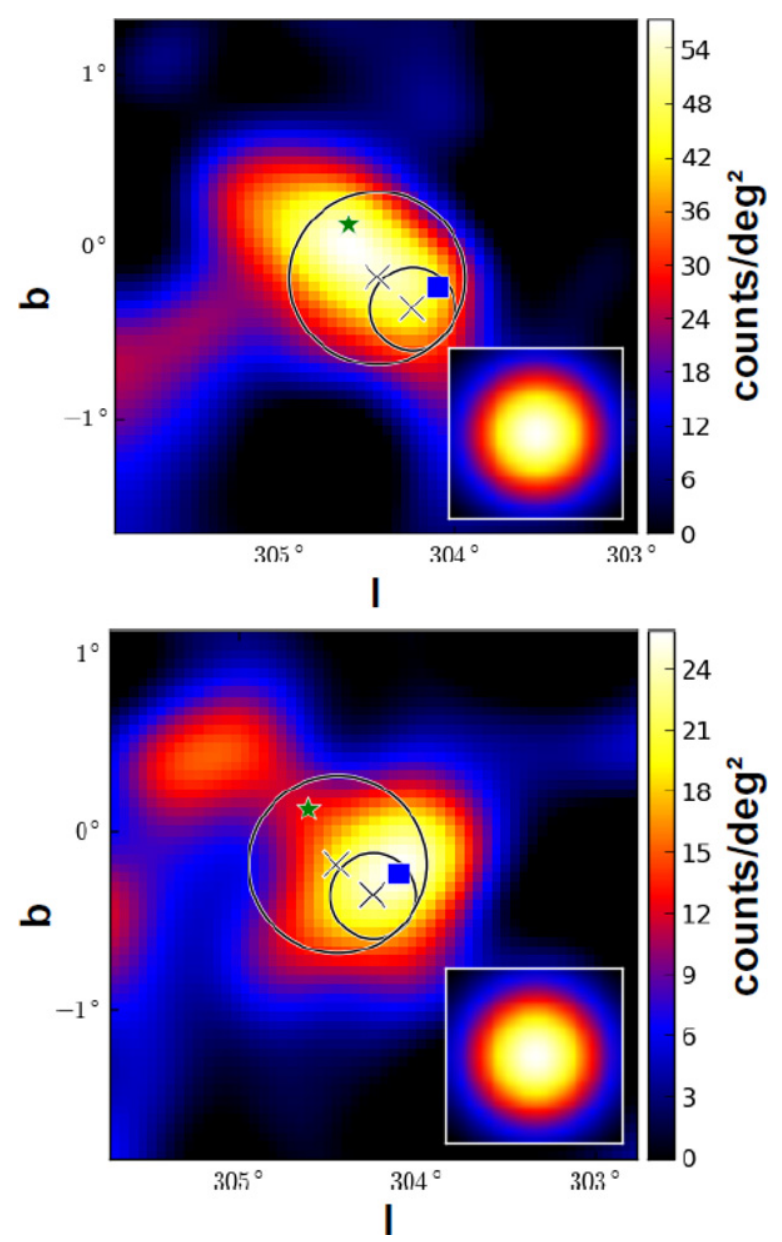

Figure 7. Count map of the region of HESS J1303-631 above $10 \mathrm{GeV}$ (top) and above $31 \mathrm{GeV}$ (bottom). We subtracted the Galactic and isotropic diffuse emission. The count map is smoothed by a Gaussian of 0.27 corresponding to the PSF above $10 \mathrm{GeV}$. The green star indicates the position of the SNR Kes 17, and the blue square represents the position of PSR J1301-6305. The small and big circles, respectively, show the extension of the H.E.S.S. Gaussian proposed by Aharonian et al. (2005c) and the extension of the Gaussian derived in this work. The lower right inset is the model-predicted emission from a point-like source with the same spectrum as HESS J1303-631 smoothed by the same kernel.

(A color version of this figure is available in the online journal.)

energy range is well below the flux detected by the Fermi-LAT. This may be due to the absence of infrared and optical photon fields in the model described above or to the contamination produced by Kes 17. A specific analysis is needed to make conclusions about the constraints that the Fermi-LAT could yield on the $\gamma$-ray emission of this source.

$$
\text { 4.5.3. HESS J1356-645 }
$$

HESS J1356-645 is an extended source detected by H.E.S.S. during the Galactic Plane Survey (H.E.S.S. Collaboration et al. 2011c). It lies close to the pulsar PSR J1357-6429 discovered during the Parkes multibeam survey of the Galactic plane (Camilo et al. 2004). Its high spin-down power of $\dot{E}=3.1 \times$ $10^{36} \mathrm{erg} \mathrm{s}^{-1}$ makes it a good candidate to power a PWN. Analysis of archival radio and X-ray data from ROSAT/PSPC and XMMNewton has revealed a faint extended structure coincident with the VHE emission (H.E.S.S. Collaboration et al. 2011c), providing another argument in favor of the PWN scenario. In parallel, Lemoine-Goumard et al. (2011b) announced the detection of a pulsed signal from PSR J1357-6429 in the $\gamma$-ray 

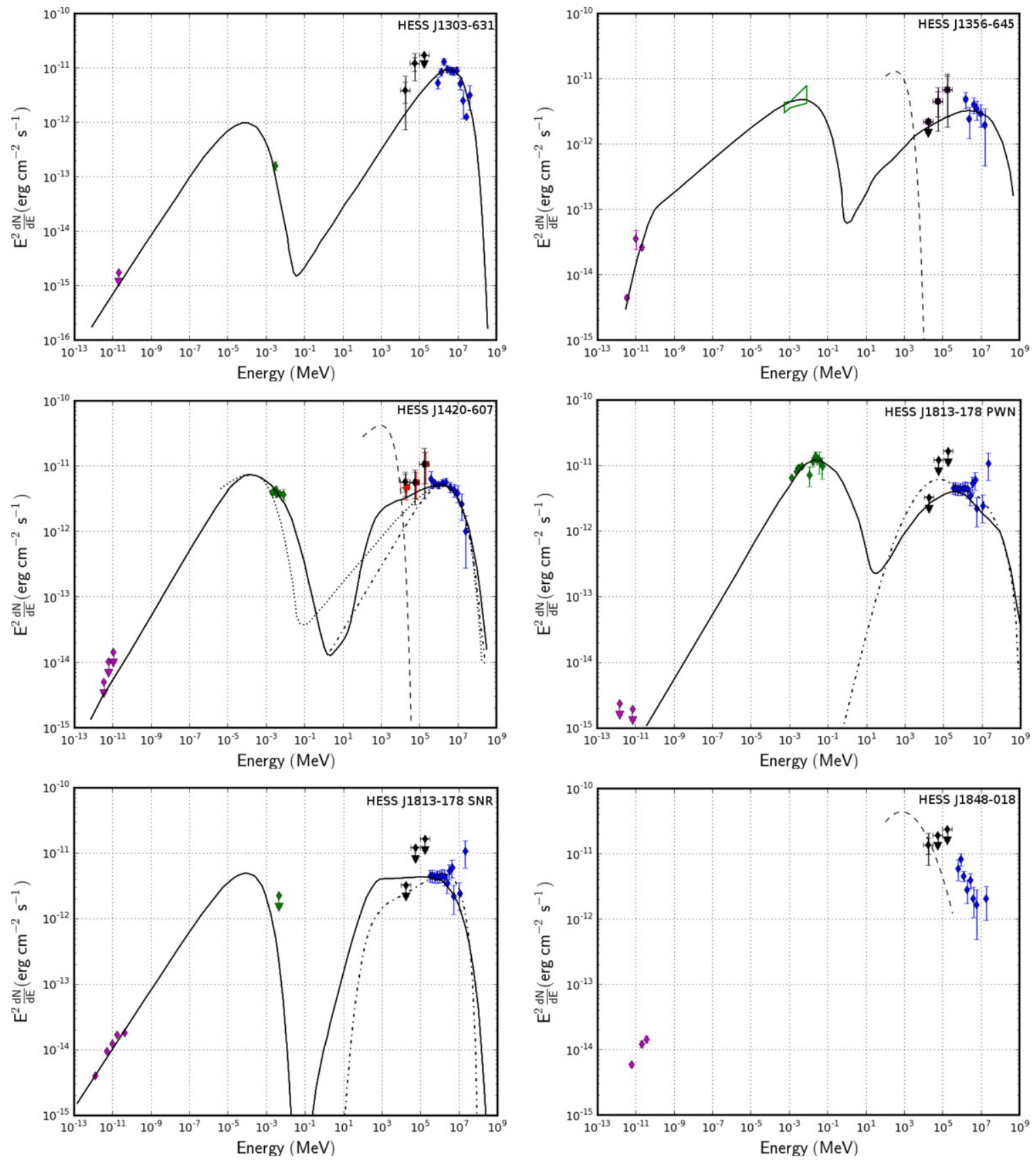

Figure 8. Multi-wavelength SEDs of HESS J1303-631, HESS J1356-645, HESS J1420-607, HESS J1813-178, and HESS J1848-018. The blue, green, and magenta points represent the H.E.S.S., X-ray, and radio spectra, respectively. The conventions used for the LAT spectral points are the same as in Figure 5. The dashed line corresponds to the model of associated pulsars. Details on the models are given in the text. The solid, dotted, and dashed-dotted lines represent multi-wavelength models from other publications. Models and references-HESS J1303-631: leptonic model proposed by H.E.S.S. Collaboration et al. (2012b). HESS J1356-645: leptonic model proposed by H.E.S.S. Collaboration et al. (2011c). HESS J1420-607: the solid and dashed lines show the hadronic+leptonic and leptonic models proposed by Van Etten \& Romani (2010), and the dotted line represents the leptonic model proposed by Kishishita et al. (2012). HESS J1813-178 PWN: the solid and dashed lines, respectively, show the leptonic models proposed by Funk et al. (2007a) and Fang \& Zhang (2010). HESS J1813-178 SNR: the solid and dashed lines, respectively, show the hadronic models proposed by Funk et al. (2007a) and Fang \& Zhang (2010).

(A color version of this figure is available in the online journal.)

and X-ray energy ranges using Fermi-LAT and XMM-Newton data. However, using 29 months of LAT data between 0.1 and $100 \mathrm{GeV}$, no counterpart to the H.E.S.S. emission was found in the off-pulse window of the pulsar.

The 16 additional months of observations by Fermi-LAT and the higher maximum energy used ( $316 \mathrm{GeV}$ instead of $100 \mathrm{GeV}$ ) in our data set now enable the detection of a faint counterpart to the H.E.S.S. emission with a TS $=24$ ( $4.7 \sigma$ assuming 2 dof).
With its spectral cutoff at low energy, $\sim 800 \mathrm{MeV}$ (LemoineGoumard et al. 2011b), PSR J1357-6429 is not significant in the $10-316 \mathrm{GeV}$ energy range. Therefore, we do not expect to see any changes in the spectral parameters when adding PSR J1357-6429 to the model of the region. This is verified in Table 5 as well as in Figure 8.

The combined LAT-H.E.S.S. data in Figure 8 provide new information concerning the spectral shape of the $\gamma$-ray emission. 
The spatial and spectral consistency between the LAT and H.E.S.S. emission suggests a physical relationship, leading to the assumption that the LAT and the H.E.S.S. emissions are due to the same object. Assuming that the $\gamma$-ray signal comes from the PWN powered by PSR J1357-6429, H.E.S.S. Collaboration et al. (2011c) proposed a leptonic scenario (black curve) which provides an excellent fit of the new multi-wavelength data. This one-zone model is based on the evolution of an electron population injected with an exponentially cutoff power-law spectrum of index 2.5 and cutoff energy of $350 \mathrm{TeV}$. These electrons cool radiatively through IC scattering on the CMB, Galactic infrared $(T \sim 35 \mathrm{~K}$ and $350 \mathrm{~K})$, and optical $(T \sim 4600 \mathrm{~K})$ photons and through synchrotron emission in a magnetic field $\sim 3.5 \mu \mathrm{G}$.

The similarities between PSR J1357-6429 and the Vela pulsar and between their PWNe led H.E.S.S. Collaboration et al. (2011c) to discuss two leptonic emission components. In the case of Vela X, the "halo" is seen in the LAT and radio energy ranges and the "cocoon" in the H.E.S.S. and X-ray energy ranges, while in the case of HESS J1356-645, a single lepton population explains the broadband spectrum with reasonable parameters. Unlike Vela X, PSR J1357-6429 is very faint in radio and X-ray, and observations in these bands do not provide morphological constraints. Future multi-wavelength data are greatly needed to better describe this source.

\subsubsection{HESS J1420-607}

The complex of compact and extended radio/X-ray sources, called Kookaburra (Roberts et al. 1999), spans over $1 \mathrm{deg}^{2}$ along the Galactic plane. It has been extensively studied to explain the EGRET source 3EG J1420-6038/GEV J1417-6100 (Hartman et al. 1999; Lamb \& Macomb 1997). Within its northeast excess, designated "K3," the young and energetic pulsar PSR J1420-6048 with period $68 \mathrm{~ms}$, characteristic age $\tau_{C}=$ $13 \mathrm{kyr}$, and spin-down power $10^{37} \mathrm{erg} \mathrm{s}^{-1}$ was discovered (D'Amico et al. 2001). X-ray observations by ASCA and later by Chandra and XMM-Newton revealed extended X-ray emission surrounding this pulsar and identified as a potential PWN (Roberts et al. 2001; $\mathrm{Ng}$ et al. 2005). On the southwest side of the Kookaburra complex lies a bright nebula exhibiting extended hard X-ray emission, G313.1+0.1 (aka the "Rabbit"; Roberts et al. 1999). This X-ray excess was also proposed as a PWN contributing to the $\gamma$-ray emission detected by EGRET.

The H.E.S.S. Galactic Plane Survey revealed two VHE sources in this region: HESS J1420-607 and HESS J1418-609 (Aharonian et al. 2006a). HESS J1420-607 is centered north of PSR J1420-6048 (near K3), while HESS J1418-609 is coincident with the Rabbit nebula. More recently, Fermi-LAT detected pulsed $\gamma$-ray emission from PSR J1420-6048 and PSR J1418-6058, the latter being a new $\gamma$-ray pulsar found through blind frequency searches (Abdo et al. 2010e, 2009a). PSR J1418-6058 is coincident with an X-ray source in the Rabbit PWN and has a spin-down power high enough to power the PWN candidate HESS J1418-609.

Figure 9 shows two smoothed count maps centered on the location of the K3 nebula. The Galactic and the isotropic diffuse emissions were subtracted to show the excesses coming from HESS J1420-607 and HESS J1418-609. Above $10 \mathrm{GeV}$, HESS J1418-609 and HESS J1420-607 are confused due to the size of the LAT PSF and to the limited statistics. However, on the count map above $31 \mathrm{GeV}$, the emission from HESS J1418-609 disappears, confirming the soft spectrum and the
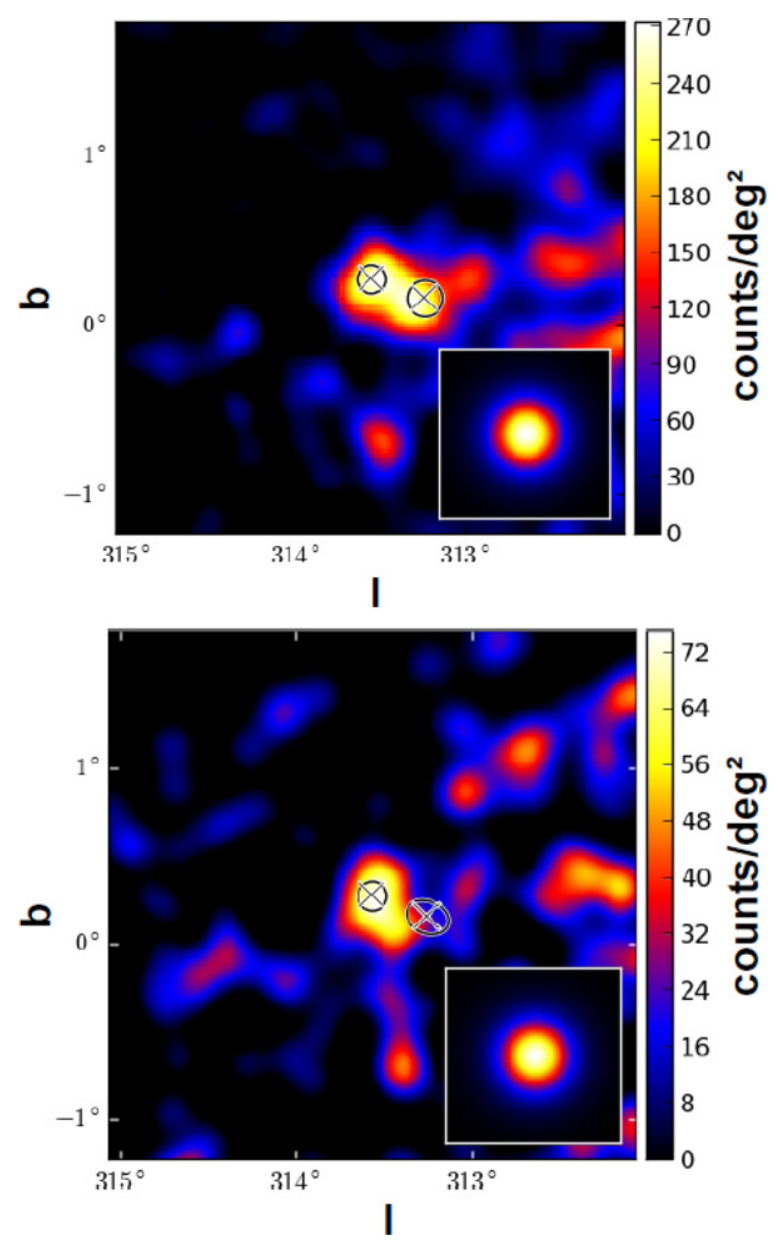

Figure 9. Smoothed count map of the region of the Kookaburra complex observed by Fermi above $10 \mathrm{GeV}$ (top) and $31 \mathrm{GeV}$ (bottom). The Galactic and isotropic diffuse emissions are subtracted. The left and right circles show the best fit obtained by VHE experiments for the K3 nebula and the Rabbit nebula, respectively. The lower right inset is the model-predicted emission from a point-like source with the same spectrum as HESS J1420-607 smoothed by the same kernel. There is significant emission from both regions for energies above $10 \mathrm{GeV}$, but there is only significant emission from HESS J1420-607 for energies above $30 \mathrm{GeV}$.

(A color version of this figure is available in the online journal.)

pulsar-like emission coming from HESS J1418-609 and the harder spectrum from HESS J1420-607.

Van Etten \& Romani (2010) used a two-zone time-dependent numerical model with constant injection luminosity to investigate the physical properties of HESS J1420-607. The authors injected relativistic particles following a power-law spectrum into the inner nebula zone; they evolved this spectrum over time and injected the resultant spectrum into the outer nebula zone. Figure 8 shows the results obtained for a hadronic + leptonic model on the assumption of a low-density environment $\left(n \sim 1 \mathrm{~cm}^{-3}\right)$ and magnetic fields of $12 \mu \mathrm{G}$ and $9 \mu \mathrm{G}$, respectively, in the inner and outer nebula. The same figure presents a leptonic scenario assuming magnetic fields of $12 \mu \mathrm{G}$ and $8 \mu \mathrm{G}$, respectively, in the inner and outer nebula. The strength of this magnetic field implies a lepton spectral break at $\sim 100 \mathrm{TeV}$ after evolution in the inner nebula. More recently, Kishishita et al. (2012) proposed a one-zone leptonic model assuming a power-law injection spectrum with an index of 2.3 with a cutoff at $\sim 40 \mathrm{TeV}$ and a magnetic field of $\sim 3 \mu \mathrm{G}$; the model SED is shown in the same figure. 
As explained in Section 3, the model used for PSR J1420-6048 was fixed to the 2FGL spectrum. Therefore, if this model does not perfectly reproduce the data with 21 additional months, the LAT spectral points of HESS J1420-608 might still be contaminated by the pulsar's emission. With the current statistics, all models reproduce the LAT and H.E.S.S. data reasonably well. A future off-pulse analysis of LAT data for this pulsar performed with more statistics could help discriminate between the models.

\subsubsection{HESS J1841-055}

HESS J1841-055 was discovered during the H.E.S.S. Galactic Plane Survey (Aharonian et al. 2008c) and remained unidentified. The emission is highly extended and shows possibly three peaks, and more than one possible counterpart, suggesting that the H.E.S.S. emission is composed of more than one source. Using INTEGRAL data, Sguera et al. (2009) proposed the highmass X-ray binary system AX J1841.0-0536 as a potential counterpart, at least for part of the emission. Nolan et al. (2012) and Neronov \& Semikoz (2012) detected three sources coincident with HESS J1841-055. Tibolla (2011) proposed the association of HESS J1841-055 with an ancient PWN powered by PSR J1841-0524, PSR J1838-0549, or both as each pulsar taken independently would need an efficiency greater than $100 \%$ to solely power a potential PWN associated with the H.E.S.S. source. More recently, the blind search detection of the new $\gamma$-ray pulsar PSR J1838-0537 with Fermi-LAT provided another potential counterpart of the H.E.S.S. source. Indeed, assuming a distance of $2 \mathrm{kpc}$, Pletsch et al. (2012) estimated that PSR J1838-0537 is sufficiently energetic to power the whole H.E.S.S. source with a conversion efficiency of $0.5 \%$, similar to other suggested pulsar/PWN associations (Hessels et al. 2008).

In this work, HESS J1841-055 is detected as a significantly extended source $\left(\mathrm{TS}_{\mathrm{ext}}=32\right)$ at a position consistent with the H.E.S.S. source. The LAT extension of $0.38 \pm 0.09$ is comparable with the $0.33 \pm 0.04$ of the H.E.S.S. source.

As can be seen in Figure 6, the Fermi-LAT spectral points connect to the H.E.S.S. ones. The spatial consistency and spectral connection between H.E.S.S. and LAT emissions suggest a physical relationship. The hard Fermi-LAT spectrum detected implies that a curvature must arise between the H.E.S.S. energy range and the LAT energy range. This is typical of most PWNe detected by Fermi-LAT and H.E.S.S. that present IC emission peaking at a few hundreds of $\mathrm{GeV}$ and would favor the PWN scenario. However, this source is very extended at both wavelengths and could be composed of several $\gamma$-ray sources. Follow-up observations with VHE experiments and continued observation with Fermi-LAT are needed to unveil the real nature of HESS J1841-055.

\subsubsection{HESS J1848-018}

HESS J1848-018 was discovered during the extended H.E.S.S. Galactic Plane Survey (Chaves et al. 2008), in the direction of, but slightly offset from, the star-forming region W43 (aka G30.8-0.2). The H.E.S.S. emission is characterized by significant extension $(0.32 \pm 0.02)$, a power-law spectrum of index $\sim 2.8$, and an integrated flux above $1 \mathrm{TeV} \sim 2 \times 10^{-12} \mathrm{~cm}^{-2} \mathrm{~s}^{-1}$. The absence of an energetic pulsar or SNR within 0.5 from HESS J1848-018 favors an association with the star-forming region W43. The only potential counterpart for this source found in radio and X-rays is the Wolf-Rayet star WR 121a.

Located 0.2 from the centroid of HESS J1848-018, WR 121a is a WN7 subtype star, in a binary system (Luque-Escamilla et al.
2011), associated with W43 and characterized by extreme massloss rates. Chaves et al. (2008) also proposed an association of the H.E.S.S. emission with the molecular clouds contained in W43. These molecular clouds could lead to the production of high-energy $\gamma$-rays from the neutral pion decays following $p-p$ collisions in the ambient gas.

In the LAT energy range, Tam et al. (2010) proposed an association with a spatially coincident source 0FGL J1848.6-0138. Lemoine-Goumard et al. (2011a) analyzed the Fermi-LAT data around HESS J1848-018 and detected a source with a $3.7 \sigma$ evidence for an extension $(\sigma \sim 0.3)$. This disfavors models in which the LAT emission would be produced by a pulsar alone. However, statistics were not large enough to discriminate between one extended source and several point sources. Moreover, the spectrum was well described by a log-normal representation (Equation (3)), and the SED was very similar to those obtained for most pulsars detected by Fermi-LAT. Therefore, the emission could be a composite of a radio-faint pulsar and an additional source.

In our analysis, HESS J1848-018 is detected as a faint point-like source but consistent with the extension reported by Lemoine-Goumard et al. (2011a). Figure 8 includes the H.E.S.S. spectral points from Chaves et al. (2008) and the radio points corresponding to the W43 central cluster from Luque-Escamilla et al. (2011). We note that the point obtained in our analysis is consistent with the dashed curve, which represents the spectrum derived by Lemoine-Goumard et al. (2011a). It is not absolutely clear from this figure whether the LAT and H.E.S.S. spectra have a common or a distinct origin, and future multi-wavelength data would be needed to discriminate between the pulsar/PWN and the massive star formation region hypotheses.

\subsection{Constraints Obtained from Non-detections}

This section presents the sources for which no $\gamma$-ray emission is detected but the corresponding upper limits constrain the models.

\subsubsection{HESS J1026-582}

HESS J1026-582 was discovered by H.E.S.S. during an improved analysis of the region of HESS J1023-575 (H.E.S.S. Collaboration et al. 2011d). While HESS J1023-575 is close to the energetic pulsar PSR J1023-5746 ( $\left.\dot{E}=10^{37} \mathrm{erg} \mathrm{s}^{-1}\right)$ discovered by the LAT using blind search algorithms (Saz Parkinson et al. 2010), the authors proposed an association between HESS J1026-582 and PSR J1028-5819 discovered by the Parkes $64 \mathrm{~m}$ telescope (Keith et al. 2008) and also detected by the LAT (Abdo et al. 2009d). The proximity of the pulsar suggested a PWN scenario to explain the VHE emission. This hypothesis is supported by the spin-down power of PSR J1028-5819, $\dot{E}=8.43 \times 10^{35} \mathrm{erg} \mathrm{s}^{-1}$ (Abdo et al. 2009d), typical of observed PWNe. Mignani et al. (2012) observed marginal X-ray emission but could not definitely classify the emission as being characteristic of a PWN. Followup observations with XMM-Newton and Chandra are needed to confirm this detection.

No significant LAT emission coming from the location of the H.E.S.S. excess is detected in our analysis. The very low TS value of 1.0 with an integrated flux less than $1.6 \times$ $10^{-10}$ photons $\mathrm{cm}^{-2} \mathrm{~s}^{-1}$ (see Table 6) gives little hope for a future detection by the LAT. The upper limits in Figure 4 show that a rising spectrum is needed in the LAT energy range. This suggests an IC peak at energies higher than $100 \mathrm{GeV}$ consistent with Fermi-LAT observations of other PWNe. However, the 
lack of multi-wavelength data (especially in radio and X-rays) prevents clear identification of this source.

\subsubsection{HESS J1458-608}

PSR J1459-6053, discovered in $\gamma$-rays, is an energetic and older pulsar with a spin-down power of $E=9.2 \times 10^{35} \mathrm{erg} \mathrm{s}^{-1}$ and a characteristic age of $\tau_{C}=64 \mathrm{kyr}$ (Abdo et al. 2010e). An X-ray counterpart to PSR J1459-6053 was discovered by Swift (Ray et al. 2011) and Suzaku (Kanai 2007). HESS J1458-608 was discovered 9'6 away from PSR J1459-6053 after a dedicated observation (de los Reyes et al. 2012). The proximity to the pulsar and the extension of HESS J1458-608 suggested that both objects could be related in a PSR/PWN scenario. In this case, the lack of the faint X-ray emission could be explained by the system's age.

In our work, HESS J1458-608 was not significantly detected above $10 \mathrm{GeV}$. Table 6 shows that the observed marginal emission comes from the energy bin between 10 and $31 \mathrm{GeV}$. Figure 4 shows that subtracting the pulsar's contribution does not change the SED. This comes from the fact that the spectrum of the pulsar in the 2FGL catalog above $10 \mathrm{GeV}$ is negligible compared to the SED. The upper limits computed in the energy bins between 31 and $316 \mathrm{GeV}$, where no pulsar emission is expected, show that a change in the slope of the spectrum is needed between the H.E.S.S. and the LAT component. This is consistent with an IC peak above $100 \mathrm{GeV}$ in the range observed for the PWNe detected with the Fermi-LAT. However, the H.E.S.S. spectrum of HESS J1458-608 differs from other PWNe, since it seems to show a hardening at high energy.

\subsubsection{HESS J1626-490}

HESS J1626-490 is another UNID source detected during the H.E.S.S. Galactic Plane Survey (Aharonian et al. 2008c). According to a leptonic model, Eger (2011) found no X-ray source bright enough to be consistent with the H.E.S.S. emission using XMM-Newton observations. However, the authors suggested that a hadronic scenario based on the interaction of SNR G335.2+00.1 with a molecular cloud could explain the H.E.S.S. emission. This hypothesis is supported by a density depression in $\mathrm{H}$ i that could be explained by a recent event such as a supernova.

With a TS of 1.5, HESS J1626-490 is not detected in our analysis. The model presented in Eger (2011) and shown in Figure 5 reproduces the H.E.S.S. SED and predicts emission below the LAT upper limits. A radio or/and X-ray detection of synchrotron emission from a PWN or the detection of a pulsar could call this model into question.

\subsubsection{HESS J1813-178}

HESS J1813-178 was discovered during the H.E.S.S. survey of the Inner Galaxy (Aharonian et al. 2005a) and also detected by MAGIC (Albert et al. 2006a). The source was identified as being SNR G12.8-0.0 following its radio detection by Brogan et al. (2005). Using XMM-Newton observations, Funk et al. (2007a) detected a complex morphology composed of a pointlike source and an extended nebula. The morphological and spectral similarities of the central object with a PWN led Funk et al. (2007a) to propose a PWN/SNR scenario to describe the $\mathrm{X}$-ray sources. This hypothesis was strengthened by the discovery of PSR J1813-1749 (Gotthelf \& Halpern 2009). This pulsar is one of the most energetic pulsars in our Galaxy with a spin-down power of $\dot{E}=5.6 \times 10^{37} \mathrm{erg} \mathrm{s}^{-1}$ but has not yet been detected by the LAT. However, the nature of the H.E.S.S. emission remains unclear as either the SNR or the PWN could produce emission at these energies.

Our analysis yielded a TS of 2.5 with an upper limit on the integrated flux of $2.4 \times 10^{-10} \mathrm{ph} \mathrm{cm}^{-2} \mathrm{~s}^{-1}$ assuming the H.E.S.S. morphology as spatial shape. Figure 8 shows the multi-wavelength SED of HESS J1813-178. The upper limits derived using the procedure described in Section 3 show that the spectrum of HESS J1813-178 cannot be flat between the H.E.S.S. and the LAT energy ranges and suggest a peak with an energy cutoff located between the two energy ranges.

Funk et al. (2007a) and Fang \& Zhang (2010) investigated a leptonic model in which the X-ray core and VHE $\gamma$-ray emission are associated. Both take into account IC scattering on $\mathrm{CMB}$, infrared and near-infrared photon fields, and synchrotron emission produced with a rather low magnetic field $(B \sim$ $7 \mu \mathrm{G})$. The main difference between these two models lies in the injected electron population, which follows a power-law spectrum with an index of 2.0 in Funk et al. (2007a) and a Maxwellian + power-law tail spectrum (Spitkovsky 2008) with an index of 2.4 in Fang \& Zhang (2010).

Both also investigated the possibility for the H.E.S.S. signal to be created by the SNR shell. We overlaid on Figure 8 the models proposed by Funk et al. (2007a) and Fang \& Zhang (2010). The main differences between these two models lie in the injected proton and electron populations which follow power-law spectra with an index of 2.1 in Funk et al. (2007a) and are computed in a semi-analytical nonlinear model in Fang \& Zhang (2010).

The upper limits derived in our analysis rejected one of the two models proposed in each hypothesis. Therefore, no conclusion can be reached on the nature of the H.E.S.S. emission being a PWN or an SNR. However, in light of the different models discussed one can exclude a model assuming a Maxwellian + power-law tail injection spectrum with an index of 2.4 with the parameters derived in Fang \& Zhang (2010) in the PWN scenario. These upper limits also constrain the hadronic model by rejecting a power-law injection spectrum of 2.1 with the parameters proposed by Funk et al. (2007a). Therefore, whatever the origin of the $\gamma$-ray emission, the injected spectrum of the primary electrons and protons needs to be relatively hard in order to stay below the Fermi-LAT upper limits $(\Gamma \leqslant 2.1)$.

\section{DISCUSSION}

In this section, we investigate the correlations of pulsar age and spin-down power with the flux of the associated PWN in the $\mathrm{keV}, \mathrm{GeV}$, and $\mathrm{TeV}$ bands. Table 7 lists the ages, spindown luminosities, and distances for the associated pulsars or other distance information, and Table 10 lists the X-ray, GeV, and TeV flux (or flux upper limit) for the associated PWNe. As a first step, we studied the relation between the spectra in the VHE energy range and the LAT energy range. We assumed that the emission measured in the $\mathrm{TeV}$ and $\mathrm{GeV}$ bands comes from the falling and rising edges, respectively, of the IC peak in the SED produced by the same population of electrons, and we studied the spectral shape of this IC peak. Mayer et al. (2012) have shown that a negative correlation between the energy of the peak and the pulsar characteristic age would be expected from the evolution of the cooling time with energy. We attempted to find this relation starting from the LAT and VHE data.

Among the 58 sources analyzed, 36 sources have an associated pulsar. We selected the 14 sources flagged as PWN or PWNc in Table 4 as they are likely due to IC emission. 
Table 10

PWN Properties

\begin{tabular}{|c|c|c|c|c|c|}
\hline Name & $\begin{array}{c}G_{10 \mathrm{GeV}}^{316 \mathrm{GeV}} \\
\left(10^{-12} \mathrm{erg} \mathrm{cm}^{-2} \mathrm{~s}^{-1}\right)\end{array}$ & $\begin{array}{c}\begin{array}{c}G_{1 \mathrm{TeV}}^{30 \mathrm{TeV}} \\
\left(10^{-12} \mathrm{erg} \mathrm{cm}^{-2} \mathrm{~s}^{-1}\right)\end{array}\end{array}$ & $\begin{array}{c}\begin{array}{c}G_{2 \mathrm{keV}}^{10 \mathrm{keV}} \\
\left(10^{-12} \mathrm{erg} \mathrm{cm}^{-2} \mathrm{~s}^{-1}\right)\end{array}\end{array}$ & $\begin{array}{c}L_{10 \mathrm{GeV}}^{316 \mathrm{GeV}} \\
\left(10^{34} \mathrm{erg} \mathrm{s}^{-1}\right)\end{array}$ & References \\
\hline VER J0006+727 & $<6.9$ & $\ldots$ & $\ldots$ & $<0.2$ & $\ldots$ \\
\hline Crab & $486 \pm 188$ & $80 \pm 17$ & $21000 \pm 4200$ & $23 \pm 15$ & (1) \\
\hline MGRO J0631+105 & $<6.0$ & $\ldots$ & $\ldots$ & $<0.1$ & $\cdots$ \\
\hline MGRO J0632+17 & $<29$ & $\ldots$ & $\ldots$ & $<0.01$ & $\cdots$ \\
\hline Vela X & $134 \pm 11$ & $79 \pm 22$ & $54 \pm 11$ & $0.14 \pm 0.02$ & (1) \\
\hline HESS J1018-589 & $6.8 \pm 6.3$ & $0.9 \pm 0.4$ & $\ldots$ & $0.7 \pm 1.0$ & (2) \\
\hline HESS J1023-575 & $27 \pm 12$ & $4.8 \pm 1.7$ & $\cdots$ & $2.5 \pm 1.1$ & (3) \\
\hline HESS J1026-582 & $<9.4$ & $5.9 \pm 4.4$ & $\cdots$ & $<0.6$ & (3) \\
\hline HESS J1119-614 & $9.1 \pm 5.2$ & $2.3 \pm 1.2^{\mathrm{a}}$ & $\ldots$ & $7.7 \pm 4.4$ & (4) \\
\hline HESS J1303-631 & $16 \pm 11$ & $27 \pm 1$ & $0.16 \pm 0.03$ & $8.3 \pm 6.6$ & (5) \\
\hline HESS J1356-645 & $16 \pm 10$ & $6.7 \pm 3.7$ & $0.06 \pm 0.01$ & $1.2 \pm 0.9$ & (6) \\
\hline HESS J1418-609 & $<25$ & $3.4 \pm 1.8$ & $3.1 \pm 0.2$ & $<0.8$ & $(7,4,8)$ \\
\hline HESS J1420-607 & $23 \pm 9$ & $15 \pm 3$ & $1.3 \pm 0.3$ & $8.6 \pm 4.5$ & $(1,7,8)$ \\
\hline HESS J1458-608 & $<15$ & $3.9 \pm 2.4$ & $\ldots$ & $<2.8$ & (9) \\
\hline HESS J1514-591 & $46 \pm 13$ & $20 \pm 4$ & $29 \pm 6$ & $10 \pm 4$ & (1) \\
\hline HESS J1554-550 & $<3$ & $1.6 \pm 0.5$ & $3 \pm 1$ & $<2$ & $(10,11)$ \\
\hline HESS J1616-508 & $46 \pm 14$ & $21 \pm 5$ & $4.2 \pm 0.8$ & $26 \pm 9$ & (1) \\
\hline HESS J1632-478 & $79 \pm 19$ & $15 \pm 5$ & $0.43 \pm 0.08$ & $8.5 \pm 2.1$ & (12) \\
\hline HESS J1640-465 & $30 \pm 11$ & $5.5 \pm 1.2$ & $0.5 \pm 0.1$ & $26 \pm 10$ & (13) \\
\hline HESS J1646-458B & $<24$ & $5 \pm 2$ & $\ldots$ & $<10$ & (14) \\
\hline HESS J1702-420 & $<26$ & $9 \pm 3$ & $0.01 \pm 0.00$ & $<7$ & (15) \\
\hline HESS J1708-443 & $29 \pm 13$ & $23 \pm 7$ & $\ldots$ & $2 \pm 1$ & (16) \\
\hline HESS J1718-385 & $<12$ & $4 \pm 2$ & $0.14 \pm 0.03$ & $<3$ & (1) \\
\hline HESS J1804-216 & $74 \pm 21$ & $12 \pm 2$ & $0.07 \pm 0.01$ & $13 \pm 5$ & (17) \\
\hline HESS J1809-193 & $<48$ & $19 \pm 6$ & $0.23 \pm 0.05$ & $<7$ & (1) \\
\hline HESS J1813-178 & $<14$ & $5.0 \pm 0.6$ & $\ldots$ & $<3.7$ & $(18,19)$ \\
\hline HESS J1818-154 & $<8.9$ & $1.3 \pm 0.9$ & $\ldots$ & $<6.5$ & (20) \\
\hline HESS J1825-137 & $59 \pm 77$ & $61 \pm 14$ & $0.4 \pm 0.1$ & $12 \pm 16$ & (1) \\
\hline HESS J1831-098 & $<11$ & $5.1 \pm 0.6$ & $\ldots$ & $<2.2$ & (21) \\
\hline HESS J1833-105 & $<12$ & $2.4 \pm 1.2$ & $40 \pm 0$ & $<3.2$ & (1) \\
\hline HESS J1837-069 & $70 \pm 23$ & $22 \pm 9$ & $0.6 \pm 0.2$ & $36 \pm 12$ & (4) \\
\hline HESS J1841-055 & $89 \pm 20$ & $24 \pm 3$ & $\ldots$ & $1.8 \pm 0.4$ & (22) \\
\hline HESS J1846-029 & $<11$ & $9 \pm 2$ & $29 \pm 1$ & $<0.2$ & (4) \\
\hline HESS J1848-018 & $30 \pm 17$ & $4 \pm 1$ & $\ldots$ & $13 \pm 7$ & (23) \\
\hline HESS J1849-000 & $<7$ & $2.1 \pm 0.4$ & $0.9 \pm 0.2$ & $<4$ & (24) \\
\hline HESS J1857+026 & $58 \pm 10$ & $18 \pm 3$ & $\ldots$ & $57 \pm 18$ & (22) \\
\hline MGRO J1908+06 & $<32$ & $12 \pm 5$ & $\ldots$ & $<4$ & (25) \\
\hline HESS J1912+101 & $<27$ & $7 \pm 4$ & $\ldots$ & $<6$ & (26) \\
\hline VER J1930+188 & $<5.5$ & $2.3 \pm 1.3$ & $5.2 \pm 0.1$ & $<5.4$ & $(4,27)$ \\
\hline VER J1959+208 & $<1.9$ & $\ldots$ & $\ldots$ & $<0.1$ & (1) \\
\hline MGRO J2019+37 & $<27$ & $\ldots$ & $\ldots$ & $<21$ & $\ldots$ \\
\hline MGRO J2228+61 & $<12$ & $\ldots$ & $0.88 \pm 0.02$ & $<7.4$ & (4) \\
\hline
\end{tabular}

Notes. The flux of the PWN candidates measured by the LAT (10-316 GeV, Column 2), by VHE experiments (1-30 TeV, Column 3), and in the 2-10 keV X-ray energy range. Column 5 is the luminosity computed assuming the pulsar distances from Table 7.

${ }^{a}$ This flux has been computed from the luminosity given in Aharonian et al. (2006d). Since no H.E.S.S. spectral index is available, we assumed a fiducial index of 2.4.

References. (1) Mattana et al. 2009, and references therein, (2) Aliu et al. 2013; (3) H.E.S.S. Collaboration et al. 2011d; (4) Kargaltsev et al. 2010; (5) H.E.S.S. Collaboration et al. 2012b; (6) H.E.S.S. Collaboration et al. 2011c; (7) Kishishita et al. 2012; (8) Aharonian et al. 2006a; (9) de los Reyes et al. 2012; (10) Temim et al. 2009; (11) Acero et al. 2012; (12) Balbo et al. 2010; (13) Aharonian et al. 2006d; (14) Abramowski et al. 2012; (15) Aharonian et al. 2006d; (16) H.E.S.S. Collaboration et al. 2011b; (17) Aharonian et al. 2006d; (18) Funk et al. 2007a; (19) Fang \& Zhang 2010; (20) Hofverberg et al. 2011; (21) Sheidaei 2011; (22) Aharonian et al. 2008c; (23) Chaves et al. 2008; (24) Terrier et al. 2008; (25) Aharonian et al. 2009; (26) Aharonian et al. 2008a; (27) Acciari et al. 2010.

We searched for a correlation between the peak energy and the characteristic age of a pulsar by estimating the peak energy of the assumed IC component. To characterize the IC peak, we used the log-normal representation,

$$
\frac{d N}{d E}=N_{0} \times\left(\frac{E}{E_{0}}\right)^{-\left[\alpha+\beta \times \log _{10}\left(\frac{E}{E_{0}}\right)\right]} .
$$

First, we checked if a log-normal spectral model was a possible representation of the spectrum of the sources observed at LAT and VHE energies. If the data were well reproduced by a log-normal representation, then there should be a correlation between the energy-flux ratio and the VHE experiment spectral index. We fixed $E_{0}$ at $300 \mathrm{GeV}$ and $\beta$ at 0.2 (since $\beta$ corresponds to the typical curvature seen in the spectra) and fitted the prefactor $N_{0}$ and the index $\alpha$ using our LAT and VHE spectral points. Figure 10 plots the LAT to VHE energy flux ratios measured assuming a power-law spectrum in each energy range as a function of their spectral indices measured by VHE experiments and the relation expected for a log-normal 


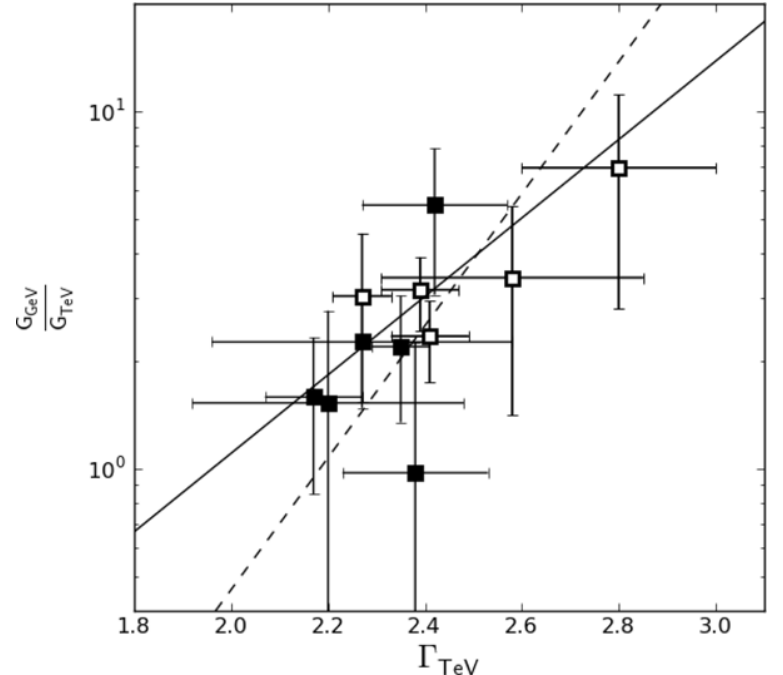

Figure 10. Ratio of the LAT to the VHE energy flux as a function of the VHE spectral index for sources classified as "PWN" or "PWNc." The LAT flux is in the $10-316 \mathrm{GeV}$ energy range, and the VHE flux is in the $1-30 \mathrm{TeV}$ energy range. The fluxes were obtained using power-law fits in each energy range. Sources classified in the TeVCat as "PWN" are represented by full markers and sources classified as "UNIDs" by hollow markers. The LAT fluxes were obtained subtracting any potential pulsar emission. The black line corresponds to a fit of a linear function to the points: $(1.10 \pm 0.48) \times \Gamma_{\mathrm{TeV}}-(2.15 \pm 1.14)$ with $\chi^{2} /$ dof $=11.2 / 9$. The dashed line corresponds to the correlation expected for a log-normal representation of $\beta=0.2$ and $E_{0}=300 \mathrm{GeV}$. HESS J1303-631 and HESS J1632-478 are not included in this figure because their energy fluxes are known to be contaminated by neighboring sources in the LAT energy range (see Section 4). HESS J1119-614 is not plotted since its TeV spectral index is not known.

model. To derive this relation, we randomly generated 1000 uniformly distributed sets of $\alpha$ providing 1000 different lognormal representations. For each log-normal model we derived the associated spectral points in $d N / d E$, assuming Poisson statistics with zero background counts, and used them to obtain the corresponding power-law index $\Gamma_{\mathrm{TeV}}$ between 1 and $30 \mathrm{TeV}$. Then the log-normal model is used to compute the energy flux ratio and derive a relation between $\Gamma_{\mathrm{TeV}}$ and this flux ratio. HESS J1303-631 and HESS J1632-478 are known to be contaminated by neighboring sources and were therefore removed from this figure. The energy flux ratio and the VHE index are correlated with a correlation coefficient of $+0.72 \pm 0.11$. Therefore, the log-normal model seems to be acceptable to reproduce the spectra between $10 \mathrm{GeV}$ and $30 \mathrm{TeV}$.

We removed HESS J1119-614 from the sample of sources since only the integrated flux above $1 \mathrm{TeV}$ is available for this source, and we required at least three spectral points to perform a fit. Then we defined the energy of the peak $\left(E_{\text {peak }}\right)$ as the energy at which the modeled SED in $v F_{\nu}$ is maximal. This also corresponds to

$$
\alpha+2 \beta \times \log _{10}\left(\frac{E_{\text {peak }}}{E_{0}}\right)=2 .
$$

Since LAT spectral points typically suffer larger uncertainty and are less numerous than the VHE ones, this method is biased by the greater weight given to the VHE side of the log-normal representation. The fit results as well as the peak position are presented in Table 11.

We plotted the resulting $E_{\text {peak }}$ as a function of the age in Figure 11. These values of $E_{\text {peak }}$ and the age yielded a correlation coefficient of $-0.06 \pm 0.28$. Furthermore, a fit of the value assuming a linear function yielded $\log _{10}\left(E_{\text {peak }} / 1 \mathrm{GeV}\right)=$

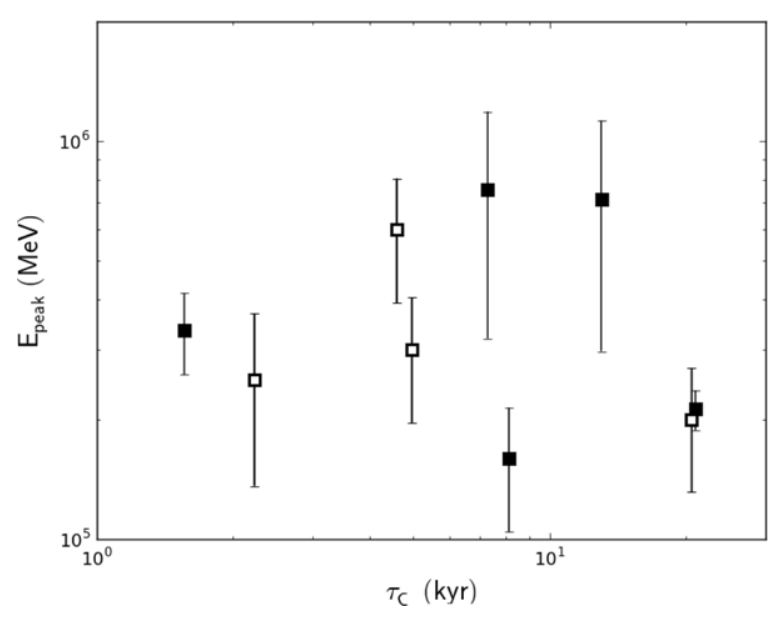

Figure 11. Energy of the maximum of the IC peak as a function of the characteristic age of the pulsar for sources labeled as PWN or PWNc in Table 4. Full markers represent sources with a clear PWN association at VHE, while hollow markers correspond to sources for which the association is less clear. The fitted SED corresponds to spectra in which contributions of pulsars summarized in Table 2 are subtracted. HESS J1640-465 and HESS J1848-018 are not plotted since the age of their putative pulsar is not known. As in Figure 10, HESS J1303-631, HESS J1632-478, and HESS J1119-614 are not plotted.

Table 11

IC Peak-fit Results

\begin{tabular}{|c|c|c|c|c|}
\hline Name & $\Gamma_{\mathrm{TeV}}$ & $\alpha$ & $\begin{array}{c}E_{\text {peak }} \\
(\mathrm{GeV})\end{array}$ & $\chi^{2} /$ dof \\
\hline HESS J1023-575 & $2.58 \pm 0.27$ & $2.17 \pm 0.07$ & $113 \pm 45$ & $9.3 / 6$ \\
\hline HESS J1303-631 a & $2.44 \pm 0$ & $1.83 \pm 0.10$ & $798 \pm 459$ & $8.2 / 9$ \\
\hline HESS J1356-645 & $2.20 \pm 0$ & $1.84 \pm 0$. & $754 \pm$ & $7.7 / 6$ \\
\hline HESS J1420-607 & $2.17 \pm 0$ & $1.88 \pm 0$ & $599 \pm$ & $8.2 / 10$ \\
\hline HESS J1514-591 & $2.27 \pm 0.31$ & $1.98 \pm 0.04$ & $337 \pm$ & $12.8 / 15$ \\
\hline HESS J1616-508 & $2.35 \pm 0$. & $2.11 \pm 0.06$ & $159 \pm$ & $4.4 / 7$ \\
\hline HESS J1632-478 & $2.12 \pm 0.20$ & $2.18 \pm 0.12$ & $106 \pm 74$ & $11.6 / 5$ \\
\hline HESS J1640-465 & $2.42 \pm 0.15$ & $2.27 \pm 0.08$ & $63 \pm 29$ & $4.9 / 6$ \\
\hline HESS J1825-137 & $2.45 \pm 0.30$ & $2.06 \pm 0.02$ & $212 \pm 24$ & $10.2 / 12$ \\
\hline HESS J1837-069 & $2.27 \pm 0.06$ & $2.03 \pm 0.08$ & $252 \pm 116$ & $11.5 / 13$ \\
\hline HESS J1841-055 & $2.41 \pm 0$ & $2.00 \pm 0.06$ & $300 \pm 103$ & $10.5 / 9$ \\
\hline HESS J1848-( & $2.8 \pm 0$ & $2.23 \pm 0.15$ & $204 \pm 112$ & $6.9 / 8$ \\
\hline HESS J1857+026 & $2.39 \pm 0.05$ & $2.07 \pm 0.06$ & $201 \pm 69$ & $9.4 / 12$ \\
\hline
\end{tabular}

Notes. The fit parameters of the log-normal fit to the LAT and VHE data. Column 2 gives the spectral index of the power-law fit by VHE experiments. Columns 3 and 4 give the index $\alpha$ at $E_{0}=300 \mathrm{GeV}$ and the peak position of the log-normal model as defined in Section 5. Column 6 gives the reduced $\chi^{2}$ of the fit. The log-normal model was fit to the VHE and LAT SED with the pulsar's contribution subtracted.

${ }^{a}$ a Since these sources are known to be contaminated by neighboring sources, the corresponding rows were not used in Section 5.

$(-0.13 \pm 0.09) \times\left(\log _{10}\left(\tau_{C} / 1 \mathrm{kyr}\right)-\overline{\log _{10}\left(\tau_{C} / 1 \mathrm{kyr}\right)}\right)+(2.41 \pm$ $0.04)$ with $\chi^{2} /$ dof $=15.9 / 7$, while a simple model assuming a constant yielded $\log _{10}\left(E_{\text {peak }} / 1 \mathrm{GeV}\right)=2.38 \pm 0.04$ with $\chi^{2} /$ dof $=16.9 / 8$. This means that the linear function improves the fit at only the $1 \sigma$ level. This coefficient and this comparison show that, contrary to the expectation (Mayer et al. 2012), no downward correlation is observed. However, this correlation might be obscured by the usage of the characteristic age of the pulsar, which may not be a good age estimator for the PWN. For instance, MSH 15-52 is a known case for which two ages are proposed, either the characteristic age of the pulsar, $1.7 \mathrm{kyr}$, or an age between 20 and $40 \mathrm{kyr}$ as suggested by the size and general appearance of the SNR (Gvaramadze 2001). 


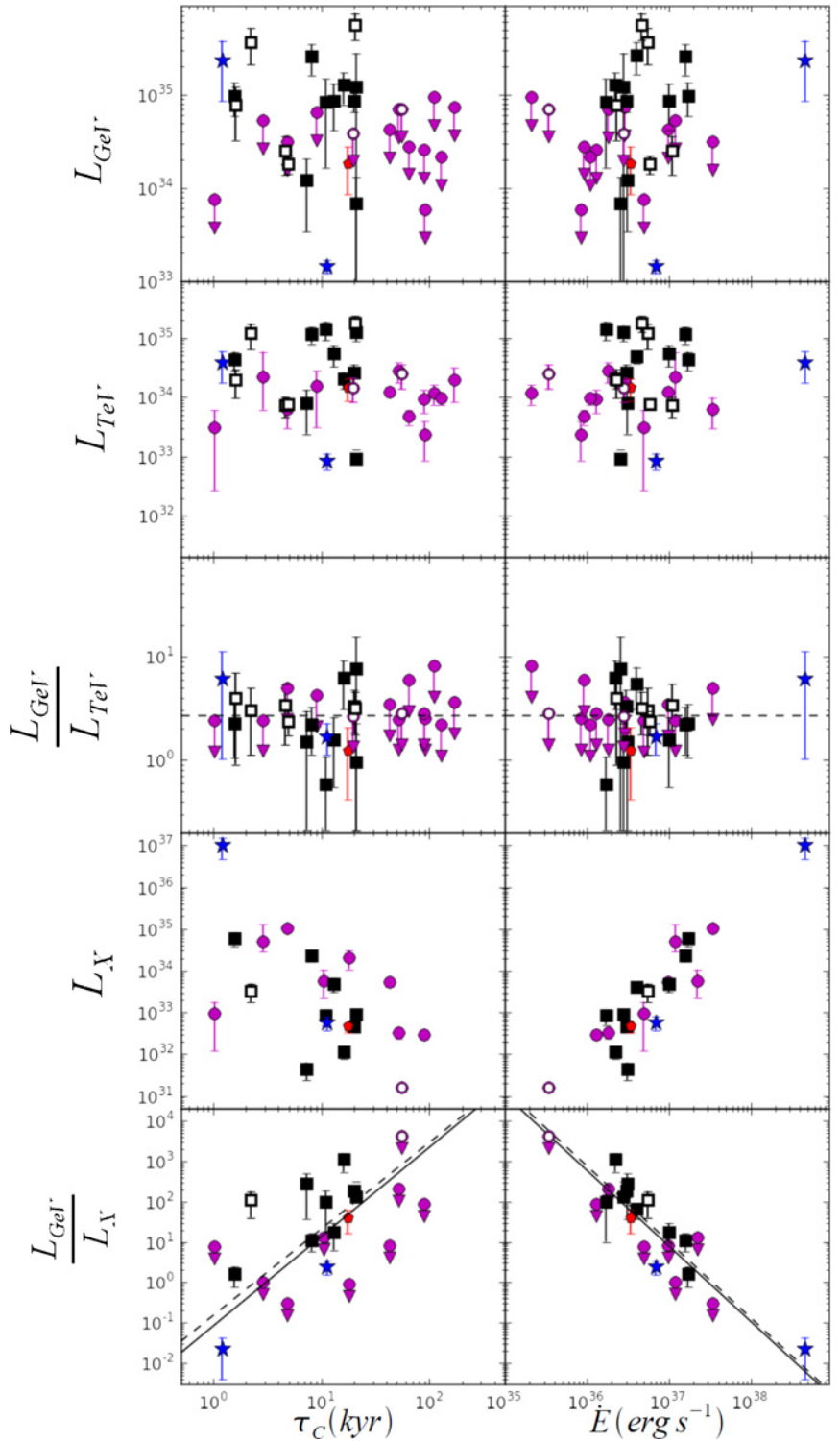

Figure 12. From top to bottom: LAT luminosity, VHE luminosity, ratio of LAT to VHE luminosity, X-ray luminosity, and the ratio of LAT to X-ray luminosity as a function of the pulsar characteristic age (left) and the pulsar spin-down power (right). The X-ray, LAT, and VHE fluxes are integrated in the $2-10 \mathrm{keV}, 10-316 \mathrm{GeV}$, and $10-30 \mathrm{TeV}$ energy ranges, respectively. Full markers correspond to sources with a clear PWN association at VHE energies, while hollow markers correspond to sources for which the association is less clear. The black squares ( $\boldsymbol{\square})$ represent the sources detected at LAT energies, the magenta circles ( $)$ show the upper limits, the red pentagon ( $)$ is HESS J1708-443 showing pulsar behavior in the LAT energy range, and the blue stars ( $\star$ ) represent the Crab Nebula and Vela X not studied in this work. The LAT luminosity corresponds to Table 10 and was computed after removing any potential emission from LAT-detected pulsars. The dashed and solid lines are explained in Section 5.

(A color version of this figure is available in the online journal.)

Using the log-normal fit presented in Table 11, we derived the mean parameters $\bar{\alpha}=2.06 \pm 0.02$ and $10^{\overline{\log _{10}\left(E_{\text {peak }}\right)}}=$ $215_{-23}^{+25} \mathrm{GeV}$. To find a putative correlation between luminosity ratio and the pulsar characteristics, we plot the GeV-to-TeV luminosity ratio as a function of the pulsar's characteristic age and spin-down energy in Figure 12. To determine if the correlation of the luminosity ratio with the characteristic age and the pulsar spin-down power is due to one of the components, this figure also presents luminosities derived in the $\mathrm{GeV}, \mathrm{TeV}$,

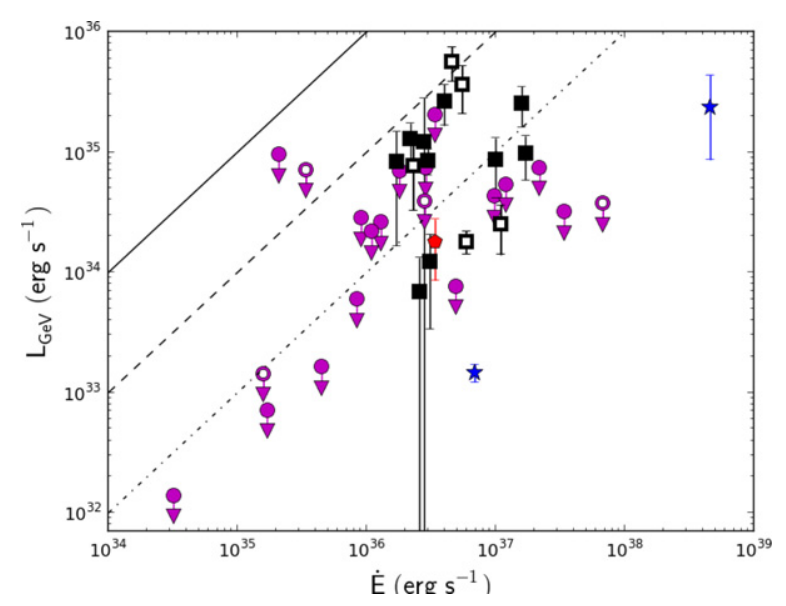

Figure 13. LAT luminosity as a function of the pulsar's spin-down power. The symbols are defined in Figure 12. The LAT luminosity was computed after removing any potential emission from LAT-detected pulsars. The upper limit on HESS J1818-154 is excluded from the plot because of its low value of $\dot{E}$.

(A color version of this figure is available in the online journal.)

and $\mathrm{keV}$ domains. We overlaid the mean ratio, $\bar{R}=10^{\overline{\log _{10}(R)}}=$ $2.7_{-1.4}^{+2.7}$, found between the LAT and VHE energy ranges using the 14 sources flagged as PWNe and PWNc in Table 4. We included in this figure and the figures that follow all sources analyzed in this work including pulsar-like sources, providing an upper limit on the PWN emission and sources flagged as "O." This figure shows that no source is located at more than $2 \sigma$ from this mean ratio except HESS J1804-216, which is not a PWN candidate as explained in Section 4.3.

As a second step, we investigated the relation between the spectra in the X-ray energy range and the LAT energy range. Mattana et al. (2009) have shown a correlation between the X-ray flux of a PWN and the physical properties of the pulsar assuming the emission of the PWN to be composed of synchrotron emission in the $\mathrm{keV}$ domain. As described in Mattana et al. (2009), the $\gamma$-ray and the X-ray luminosities of PWNe are expected to decrease with time, and the cooling time of electrons emitting synchrotron X-rays is smaller than the cooling time of the electrons emitting IC $\gamma$-rays. Therefore, the X-ray emission traces the recent evolution of a PWN, while the $\gamma$-ray photons trace a longer period. Since the pulsar spindown power driving the electron injection decreases with time, the ratio of the IC emission to the synchrotron emission is expected to increase over time.

Figure 12 and Table 10 give the ratio of the $\gamma$-ray luminosity to the X-ray luminosity as a function of the age and as a function of the pulsar spin-down power. As reported for VHE experiments (Mattana et al. 2009), no clear correlation is found between the $\gamma$-ray flux and spin-down power or between the $\gamma$-ray flux and the characteristic age. This can also be seen in Figure 13. On the other hand, there is a correlation between the X-ray flux of the observed PWNe and the pulsars' spin-down power and characteristic age. This causes the correlation between the ratio of the $\gamma$-ray flux to the X-ray flux and the pulsar properties. We also represented the relations derived in Mattana et al. (2009) multiplied by $\bar{R}$ for the whole sample of sources and for the sources clearly identified with PWNe. The overall agreement with these relations is relatively good.

In the plot showing the LAT-to-X-ray energy flux ratio as a function of the pulsar characteristic age, four upper limits are well below the correlation relations derived by Mattana et al. 
(2009). These outliers are, in increasing spin-down power, HESS J1833-105, HESS J1554-550, HESS J1849-000, and HESS J1718-385. HESS J1833-105 and HESS J1718-385 are also included in Mattana et al. (2009), unlike HESS J1554-550 and HESS J1849-000. Table 10 shows that the GeV over TeV upper limit is in each case larger than $\bar{R}$. Therefore, their small LAT/X-ray luminosity ratios do not come from an especially small LAT flux but instead from an especially large X-ray flux relative to other sources with a similar characteristic age.

These figures show that the Fermi-LAT mainly detects young and middle-aged PWNe (1-30 kyr) around energetic pulsars with spin-down powers between $10^{36}$ and $10^{39} \mathrm{erg} \mathrm{s}^{-1}$. One can see from these figures that the VHE sources older than $30 \mathrm{kyr}$ are not detected by the LAT, while Mayer et al. (2012) predict a higher flux in the LAT energy range than in the VHE experiments' energy range. However, it should be noted that among these nine VHE sources, at least seven suffer one or several biases.

1. Source misidentification. For example, HESS J1912+101 has recently been proposed to be a shell-type $\mathrm{SNR}$, 6 but some of the emission could originate in the pulsar wind. Similarly, HESS J1702-420 and HESS J1646-458B cannot be clearly associated as PWNe, and deeper multi-wavelength observations are needed to classify the emission.

2. Potential characteristic age overestimation. See, for example, HESS J1809-193 (Sheidaei 2011).

3. HESS J1026-582, HESS J1809-193, HESS J1831-098, and HESS J1849-000 are located in regions of strong diffuse emission where the LAT is less sensitive to any potential emission.

Therefore, the lack of a population of old $\gamma$-ray-loud PWNe cannot be proven given the current statistics and systematics associated with this analysis.

Finally, Figure 13 shows the $\gamma$-ray luminosity of the PWN as a function of the pulsar spin-down power assuming the distances listed in Table 7. The $\gamma$-ray luminosity is, for all sources, computed by subtracting any potential emission from nearby LAT-detected pulsars. The distance uncertainties are calculated assuming a $20 \%$ uncertainty on the dispersion measure (2PC). For most pulsars this yields a realistic uncertainty; for many, however, it leads to a large factor, for instance a factor of six in Abdo et al. (2009c), between two distance estimates. Among the detected sources, eight show a $\gamma$-ray efficiency below $1 \%$ and five are consistent within uncertainties of having an efficiency between $1 \%$ and $10 \%$. Six upper limits on the LAT luminosity of VHE sources associated with a PWN powered by an energetic pulsar $\left(\dot{E} \geqslant 10^{36} \mathrm{erg} \mathrm{s}^{-1}\right)$ are well below an efficiency of 1\%. They are HESS J1418-09, HESS 1813-178, HESS J1833-105, HESS J1849-000, MGRO J2228 + 61, and VER J1930 + 188 .

\section{CONCLUSION}

We looked for LAT counterparts to the VHE sources potentially associated with PWNe using 45 months of Fermi-LAT observations. We detected 30 of the 58 sources at LAT energies.

1. Nine may be due to pulsar emission for energies above $10 \mathrm{GeV}$.

\footnotetext{
68 This was proposed in a presentation at the AstroParticules et Cosmologie laboratory: http://www.apc.univ-paris7.fr/ semikoz/CosmicRays/ CosmicRays/Dec14/djannati-atai.pdf.
}

2. Seven sources cannot be clearly associated with a PWN.

3. Eleven are PWN candidates.

4. Three are clearly identified as PWNe: HESS J1825-137 and HESS J1514-591 (aka MSH 15-52) already detected and HESS J1356-645 detected for the first time in this analysis.

Among these 30 sources, 23 were also detected in 1FHL and 15 in Neronov \& Semikoz (2012). We analyzed their morphology and found that eight of them are significantly extended.

Adding the Crab Nebula and Vela $X$ to the three clearly identified PWNe, five PWNe are now detected at LAT energies. Eleven sources are promising PWN candidates such as HESS J1420-607 and HESS J1119-614. These 16 sources are associated with young (with an age between 1 and $30 \mathrm{kyr}$ ) and powerful pulsars with a spin-down power between $10^{36}$ and $10^{39} \mathrm{erg} \mathrm{s}^{-1}$ and typically have a conversion efficiency below $10 \%$. No correlation has been found between the LAT energy flux and the pulsar characteristic age. This work has not shown any evidence for a shift of the PWN IC emission toward the LAT energy range as a function of the characteristic age. However, this can be due to large uncertainties on the systems' age. The correlation of the LAT-to-X-ray luminosity ratio with the pulsar characteristic age and its spin-down power is consistent with the work of Mattana et al. (2009) derived for the VHE-to-X-ray luminosity ratio.

The Fermi LAT Collaboration acknowledges generous ongoing support from a number of agencies and institutes that have supported both the development and the operation of the LAT as well as scientific data analysis. These include the National Aeronautics and Space Administration and the Department of Energy in the United States; the Commissariat à l'Energie Atomique and the Centre National de la Recherche Scientifique/Institut National de Physique Nucléaire et de Physique des Particules in France; the Agenzia Spaziale Italiana and the Istituto Nazionale di Fisica Nucleare in Italy; the Ministry of Education, Culture, Sports, Science and Technology (MEXT), High Energy Accelerator Research Organization (KEK), and Japan Aerospace Exploration Agency (JAXA) in Japan; and the K. A. Wallenberg Foundation, the Swedish Research Council, and the Swedish National Space Board in Sweden.

Additional support for science analysis during the operations' phase is gratefully acknowledged from the Istituto Nazionale di Astrofisica in Italy and the Centre National d'Études Spatiales in France.

This research made use of pywcsgrid2, an open-source plotting package for Python. ${ }^{69}$ The authors acknowledge the use of the TeV catalog Web site ${ }^{70}$ provided by the University of Chicago. The authors acknowledge the use of the Australia Telescope National Facility pulsar catalog. ${ }^{71}$

\section{REFERENCES}

Abdo, A. A., Ackermann, M., Ajello, M., et al. 2009a, Sci, 325, 840 Abdo, A. A., Ackermann, M., Ajello, M., et al. 2009b, ApJ, 706, 1331 Abdo, A. A., Ackermann, M., Ajello, M., et al. 2009c, ApJ, 700, 1059 Abdo, A. A., Ackermann, M., Ajello, M., et al. 2010a, ApJ, 714, 927 Abdo, A. A., Ackermann, M., Ajello, M., et al. 2010b, ApJ, 708, 1254

\footnotetext{
69 pywcsgrid 2 can be obtained from: http://leejjoon.github.com/pywcsgrid2/.

70 The TeV catalog can be obtained from: http://tevcat.uchicago.edu/

71 The ATNF catalog can be obtained from:

http://www.atnf.csiro.au/people/pulsar/psrcat/
} 
Abdo, A. A., Ackermann, M., Ajello, M., et al. 2010c, ApJ, 713, 146 Abdo, A. A., Ackermann, M., Ajello, M., et al. 2010d, ApJ, 711, 64 Abdo, A. A., Ackermann, M., Ajello, M., et al. 2010e, ApJS, 187, 460 Abdo, A. A., Ackermann, M., Atwood, W. B., et al. 2009d, ApJL, 695, L72 Abdo, A. A., Ackermann, M., Atwood, W. B., et al. 2009e, ApJ, 696, 1084 Abdo, A. A., Allen, B. T., Aune, T., et al. 2009f, ApJL, 700, L127 Abdo, A. A., Allen, B., Berley, D., et al. 2007, ApJL, 664, L91 Abdo, A. A., \& for the Fermi-LAT Collaboration 2013, arXiv:1305.4385 Abramowski, A., Acero, F., Aharonian, F., et al. 2012, A\&A, 537, A114 Acciari, V. A., Aliu, E., Arlen, T., et al. 2010, ApJL, 719, L69 Acero, F., Djannati-Ataï, A., Förster, A., et al. 2012, arXiv:1201.0481 Ackermann, M., Ajello, M., Albert, A., et al. 2012, ApJS, 203, 4 Ackermann, M., Ajello, M., Baldini, L., et al. 2011, ApJ, 726, 35 Aharonian, F., Akhperjanian, A. G., Anton, G., et al. 2009, A\&A, 499, 723 Aharonian, F., Akhperjanian, A. G., Aye, K.-M., et al. 2005a, Sci, 307, 1938 Aharonian, F., Akhperjanian, A. G., Aye, K.-M., et al. 2005b, A\&A, 435, L17 Aharonian, F., Akhperjanian, A. G., Aye, K.-M., et al. 2005c, A\&A, 439, 1013 Aharonian, F., Akhperjanian, A. G., Aye, K.-M., et al. 2005d, A\&A, 432, L25 Aharonian, F., Akhperjanian, A. G., Barres de Almeida, U., et al. 2008a, A\&A, 484,435

Aharonian, F., Akhperjanian, A. G., Barres de Almeida, U., et al. 2008b, A\&A, 483,509

Aharonian, F., Akhperjanian, A. G., Barres de Almeida, U., et al. 2008c, A\&A, 477,353

Aharonian, F., Akhperjanian, A. G., Bazer-Bachi, A. R., et al. 2006a, A\&A, 456,245

Aharonian, F., Akhperjanian, A. G., Bazer-Bachi, A. R., et al. 2006b, A\&A, 460,365

Aharonian, F., Akhperjanian, A. G., Bazer-Bachi, A. R., et al. 2006c, A\&A, 457,899

Aharonian, F., Akhperjanian, A. G., Bazer-Bachi, A. R., et al. 2006d, ApJ, 636, 777

Aharonian, F., Akhperjanian, A. G., Bazer-Bachi, A. R., et al. 2007, A\&A, 472,489

Ajello, M., Allafort, A., Baldini, L., et al. 2012, ApJ, 744, 80

Albert, J., Aliu, E., Anderhub, H., et al. 2006a, ApJL, 637, L41

Albert, J., Aliu, E., Anderhub, H., et al. 2006b, ApJL, 643, L53

Aleksić, J., Alvarez, E. A., Antonelli, L. A., et al. 2012, APh, 35, 435

Aliu, E. 2011, International Cosmic Ray Conference, Vol. 7, 227

Aliu, E., Archambault, S., Arlen, T., et al. 2013, ApJ, 764, 38

Aliu, E., Arlen, T., Aune, T., et al. 2011, Sci, 334, 69

Atkins, R., Benbow, W., Berley, D., et al. 2003, ApJ, 595, 803

Atwood, W. B., Abdo, A. A., Ackermann, M., et al. 2009, ApJ, 697, 1071

Balbo, M., Saouter, P., Walter, R., et al. 2010, A\&A, 520, A111

Bartoli, B., Bernardini, P., Bi, X. J., et al. 2012, ApJL, 745, L22

Brogan, C. L., Gaensler, B. M., Gelfand, J. D., et al. 2005, ApJL, 629, L105

Brun, F., de Naurois, M., Hofmann, W., et al. 2011, arXiv:1104.5003

Buehler, R., Scargle, J. D., Blandford, R. D., et al. 2012, ApJ, 749, 26

Camilo, F., Bell, J. F., Manchester, R. N., et al. 2001, ApJL, 557, L51

Camilo, F., Kaspi, V. M., Lyne, A. G., et al. 2000, ApJ, 541, 367

Camilo, F., Manchester, R. N., Lyne, A. G., et al. 2004, ApJL, 611, L25

Camilo, F., Ransom, S. M., Gaensler, B. M., et al. 2006, ApJ, 637, 456

Caswell, J. L., McClure-Griffiths, N. M., \& Cheung, M. C. M. 2004, MNRAS, 352,1405

Chaves, R. C. G., Renaud, M., Lemoine-Goumard, M., \& Goret, P. 2008, in AIP Conf. Proc. 1085, High Energy Gamma-ray Astronomy, ed. F. A. Aharonian, W. Hofmann, \& F. Rieger (Melville, NY: AIP), 372

Coe, M. J., Di Mille, F., Edwards, P. G., et al. 2012, Sci, 335, 189

Cordes, J. M., \& Lazio, T. J. W. 2002, arXiv:astro-ph/0207156

D'Amico, N., Kaspi, V. M., Manchester, R. N., et al. 2001, ApJL, 552, L45

de Jager, O. C., \& Djannati-Ataï, A. 2009, in Neutron Stars and Pulsars, Astrophysics and Space Science Library, Vol. 357 (Berlin: Springer), 451

de Jager, O. C., Ferreira, S. E. S., Djannati-Ataï, A., et al. 2009, arXiv:0906.2644 de los Reyes, R., Zajczyk, A., Chaves, R. C. G., \& for the H.E.S.S. Collaboration. 2012, arXiv:1205.0719

Djannati-Ată, A., de Jager, O. C., Terrier, R., et al. 2008, in International Cosmic Ray Conference, Vol. 2, 823

Dodson, R., Legge, D., Reynolds, J. E., \& McCulloch, P. M. 2003, ApJ, 596,1137

Domainko, W., \& Ohm, S. 2012, A\&A, 545, A94

Eger, P. 2011, in International Cosmic Ray Conference, Vol. 7, 44

Fang, J., \& Zhang, L. 2010, ApJ, 718, 467

Funk, S., Hinton, J. A., Moriguchi, Y., et al. 2007a, A\&A, 470, 249

Funk, S., Hinton, J. A., Pühlhofer, G., et al. 2007b, ApJ, 662, 517

Gabici, S., Aharonian, F. A., \& Casanova, S. 2009, MNRAS, 396, 1629

Gast, H., Brun, F., Carrigan, S., et al. 2012, arXiv:1204.5860

Gonzalez, M., \& Safi-Harb, S. 2003, ApJL, 591, L143
Górski, K. M., Hivon, E., Banday, A. J., et al. 2005, ApJ, 622, 759

Gotthelf, E. V., \& Halpern, J. P. 2008, ApJ, 681, 515

Gotthelf, E. V., \& Halpern, J. P. 2009, ApJL, 700, L158

Gotthelf, E. V., Halpern, J. P., Terrier, R., \& Mattana, F. 2011, ApJL, 729, L16

Grondin, M.-H., Fermi-LAT Collaboration, \& Pulsar Timing Consortium 2013, ApJ, submitted

Grondin, M.-H., Funk, S., Lemoine-Goumard, M., et al. 2011, ApJ, 738, 42

Guillemot, L., Johnson, T. J., Venter, C., et al. 2012, ApJ, 744, 33

Gvaramadze, V. V. 2001, A\&A, 374, 259

Hall, T. A., Bond, I. H., Bradbury, S. M., et al. 2003, ApJ, 583, 853

Hartman, R. C., Bertsch, D. L., Bloom, S. D., et al. 1999, ApJS, 123, 79

H.E.S.S. Collaboration, Abramowski, A., Acero, F., et al. 2011a, A\&A, 531, A81

H.E.S.S. Collaboration, Abramowski, A., Acero, F., et al. 2011b, A\&A, 528, A 143

H.E.S.S. Collaboration, Abramowski, A., Acero, F., et al. 2011c, A\&A, 533, A103

H.E.S.S. Collaboration, Abramowski, A., Acero, F., et al. 2011d, A\&A, 525, A46

H.E.S.S. Collaboration, Abramowski, A., Acero, F., et al. 2012a, A\&A, 541, A5

H.E.S.S. Collaboration, Abramowski, A., Acero, F., et al. 2012b, A\&A, 548, A46

H.E.S.S. Collaboration, Acero, F., Aharonian, F., et al. 2011e, A\&A, 525, A45

Hessels, J. W. T., Nice, D. J., Gaensler, B. M., et al. 2008, ApJL, 682, L41

Hessels, J. W. T., Roberts, M. S. E., Ransom, S. M., et al. 2004, ApJ, 612,389

Hinton, J. A., \& Hofmann, W. 2009, ARA\&A, 47, 523

Hofverberg, P. 2011, in International Cosmic Ray Conference, Vol. 7, 247

Hofverberg, P., Chaves, R. C. G., Méhault, J., de Naurois, M., \& for the H.E.S.S. Collaboration. 2011, arXiv:1112.2901

Hoppe, S. 2008, in International Cosmic Ray Conference, Vol. 2, 579

Huang, R. H. H., Kong, A. K. H., Takata, J., et al. 2012, ApJ, 760, 92

James, F., \& Roos, M. 1975, CoPhC, 10, 343

Johnston, S., Manchester, R. N., Lyne, A. G., Kaspi, V. M., \& D’Amico, N. 1995, A\&A, 293, 795

Kanai, Y. 2007, PhD thesis, Tokyo Institute of Technology

Kargaltsev, O., Rangelov, B., \& Pavlov, G. G. 2013, arXiv:1305.2552

Keith, M. J., Johnston, S., Kramer, M., et al. 2008, MNRAS, 389, 1881

Kerr, M. 2011, PhD thesis, Univ. Washington

Kishishita, T., Bamba, A., Uchiyama, Y., Tanaka, Y., \& Takahashi, T. 2012, ApJ, 750,162

Komin, N., Djannati-Ataï, A., Gallant, Y., et al. 2012, arXiv:1201.0639

Kothes, R., Uyaniker, B., \& Pineault, S. 2001, ApJ, 560, 236

Kramer, M., Bell, J. F., Manchester, R. N., et al. 2003, MNRAS, 342, 1299

Lamb, R. C., \& Macomb, D. J. 1997, ApJ, 488, 872

Lande, J., Ackermann, M., Allafort, A., et al. 2012, ApJ, 756, 5

Leahy, D. A., Tian, W., \& Wang, Q. D. 2008, AJ, 136, 1477

Lemiere, A., Slane, P., Gaensler, B. M., \& Murray, S. 2009, ApJ, 706, 1269

Lemoine-Goumard, M., Ferrara, E., Grondin, M.-H., Martin, P., \& Renaud, M. 2011a, MmSAI, 82, 739

Lemoine-Goumard, M., Zavlin, V. E., Grondin, M.-H., et al. 2011b, A\&A, 533, A102

Lorimer, D. R., Faulkner, A. J., Lyne, A. G., et al. 2006, MNRAS, 372, 777

Lucke, P. B. 1978, A\&A, 64, 367

Luque-Escamilla, P. L., Muñoz-Arjonilla, A. J., Sánchez-Sutil, J. R., et al. 2011, A\&A, 532, A92

Manchester, R. N., Hobbs, G. B., Teoh, A., \& Hobbs, M. 2005a, yCat, 7245, 0

Manchester, R. N., Hobbs, G. B., Teoh, A., \& Hobbs, M. 2005b, AJ, 129, 1993

Manchester, R. N., Lyne, A. G., Camilo, F., et al. 2001, MNRAS, 328, 17

Mattana, F., Falanga, M., Götz, D., et al. 2009, ApJ, 694, 12

Mayer, M. 2010, Diplomarbeit: Erlangen Centre for Astroparticle Physics Physikalisches Institut 2 Lehrstuhl für Physik Friedrich-AlexanderUniversität Erlangen-Nürnberg

Mayer, M., Brucker, J., Jung, I., Valerius, K., \& Stegmann, C. 2012, arXiv: 1202.1455

Mignani, R. P., Razzano, M., Esposito, P., et al. 2012, A\&A, 543, A130

Mukherjee, R., \& Halpern, J. P. 2005, ApJ, 629, 1017

Neronov, A., \& Semikoz, D. V. 2012, PhRvD, 85, 083008

Ng, C.-Y., Roberts, M. S. E., \& Romani, R. W. 2005, ApJ, 627, 904

Nolan, P. L., Abdo, A. A., Ackermann, M., et al. 2012, ApJS, 199, 31

Paneque, D., Ballet, J., Burnett, T., et al. 2013, arXiv:1304.4153

Parent, D., Kerr, M., den Hartog, P. R., et al. 2011, ApJ, 743, 170

Pineault, S., Landecker, T. L., Madore, B., \& Gaumont-Guay, S. 1993, AJ, 105,1060

Pletsch, H. J., Guillemot, L., Allen, B., et al. 2012, ApJL, 755, L20

Ray, P. S., Kerr, M., Parent, D., et al. 2011, ApJS, 194, 17 
Renaud, M., Goret, P., \& Chaves, R. C. G. 2008, in AIP Conf. Proc. 1085, High Energy Gamma-ray Astronomy, ed. F. A. Aharonian, W. Hofmann, \& F. Rieger (Melville, NY: AIP), 281

Roberts, M. S. E., Romani, R. W., \& Johnston, S. 2001, ApJL, 561, L187

Roberts, M. S. E., Romani, R. W., Johnston, S., \& Green, A. J. 1999, ApJ, 515,712

Rousseau, R., Grondin, M.-H., Van Etten, A., et al. 2012, A\&A, 544, A3

Rowell, G., Horns, D., Fukui, Y., \& Moriguchi, Y. 2008, in AIP Conf. Proc. 1085,

High Energy Gamma-ray Astronomy, ed. F. A. Aharonian, W. Hofmann, \& F. Rieger (Melville, NY: AIP), 241

Ruiz, M. T., \& May, J. 1986, ApJ, 309, 667

Ryter, C., Cesarsky, C. J., \& Audouze, J. 1975, ApJ, 198, 103

Safi-Harb, S., \& Kumar, H. S. 2008, ApJ, 684, 532

Sakai, M., Yajima, Y., \& Matsumoto, H. 2011, PASJ, 63, 879

Saz Parkinson, P. M., Dormody, M., Ziegler, M., et al. 2010, ApJ, 725,571

Seward, F. D., Kearns, K. E., \& Rhode, K. L. 1996, ApJ, 471, 887

Sguera, V., Romero, G. E., Bazzano, A., et al. 2009, ApJ, 697, 1194

Sheidaei, F. 2011, in International Cosmic Ray Conference, Vol. 7, 243

Slane, P., Castro, D., Funk, S., et al. 2010, ApJ, 720, 266

Spitkovsky, A. 2008, ApJL, 682, L5

Sun, M., Wang, Z.-R., \& Chen, Y. 1999, ApJ, 511, 274

Tam, P. H. T., Wagner, S. J., Tibolla, O., \& Chaves, R. C. G. 2010, A\&A, 518, A 8
Tamura, K., Kawai, N., Yoshida, A., \& Brinkmann, W. 1996, PASJ, 48, L33 Temim, T., Slane, P., Gaensler, B. M., Hughes, J. P., \& Van Der Swaluw, E. 2009, ApJ, 691, 895

Terrier, R., Mattana, F., Djannati-Atai, A., et al. 2008, in AIP Conf. Proc. 1085, High Energy Gamma-ray Astronomy, ed. F. A. Aharonian, W. Hofmann, \& F. Rieger (Melville, NY: AIP), 312

Tibolla, O. 2011, in International Cosmic Ray Conference, Vol. 6, 197

Tibolla, O., Komin, N., Kosack, K., \& Naumann-Godo, M. 2008, in AIP Conf. Proc. 1085, High Energy Gamma-ray Astronomy, ed. F. A. Aharonian, W. Hofmann, \& F. Rieger (Melville, NY: AIP), 249

Tibolla, O., Vorster, M., de Jager, O., et al. 2012, in AIP Conf. Proc. 1505, High Energy Gamma-ray Astronomy, ed. F. A. Aharonian, W. Hofmann, \& F. M Rieger (Melville, NY: AIP), 349

Trimble, V. 1973, PASP, 85, 579

Uchiyama, Y. 2011, arXiv:1104.1197

Van Etten, A., \& Romani, R. W. 2010, ApJ, 711, 1168

Verbiest, J. P. W., Weisberg, J. M., Chael, A. A., Lee, K. J., \& Lorimer, D. R. 2012, ApJ, 755, 39

Weekes, T. C., Badran, H., Biller, S. D., et al. 2002, APh, 17, 221

Weinstein, A. 2009, arXiv:0912.4492

Weltevrede, P., Abdo, A. A., Ackermann, M., et al. 2010, ApJ, 708, 1426

Wilks, S. 1938, Ann. Math. Stat., 9, 60

Wu, J. H. K., Wu, E. M. H., Hui, C. Y., et al. 2011, ApJL, 740, L12

Yadigaroglu, I.-A., \& Romani, R. W. 1997, ApJ, 476, 347 